Aus der Klinik für Klinische Neurophysiologie

(Prof. Dr. med. W. Paulus)

der Medizinischen Fakultät der Universität Göttingen

\title{
Die Beeinflussung des instrumentellen \\ Lernens durch Placebo- und \\ Noceboeffekte
}

\author{
INAUGURAL-DISSERTATION \\ zur Erlangung des Doktorgrades \\ für Zahnheilkunde \\ der Medizinischen Fakultät der \\ Georg-August-Universität zu Göttingen
}

vorgelegt von

Luisa Gunkel

aus

Heilbad Heiligenstadt

Göttingen 2016 
Dekan:

Referentin:

Ko-Referentin:

Drittreferentin:

Promotor- Vertretung
Prof. Dr. rer. nat. H.K. Kroemer

apl. Prof. Dr. rer. nat. A. Antal

Prof. Dr. Melanie Wilke

Prof. Dr. Nicole Steinbüchel- Rheinwall

Prof. Dr. Rainer Mausberg

Datum der mündlichen Prüfung: 02.10.2017 
Hiermit erkläre ich, die Dissertation mit dem Titel "Die Beeinflussung des instrumentellen Lernens durch Placebo- und Noceboeffekte" eigenständig angefertigt und keine anderen als die von mir angegebenen Quellen und Hilfsmittel verwendet zu haben.

Göttingen, den 05.07.2016 


\section{Inhaltsverzeichnis}

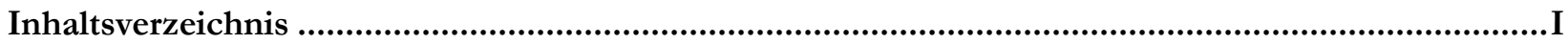

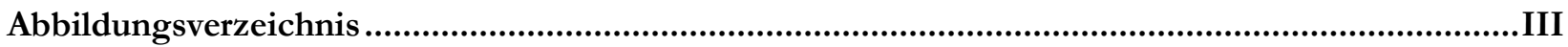

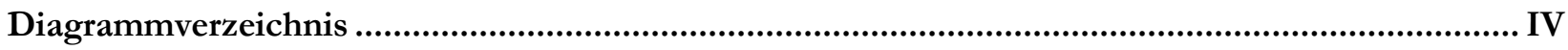

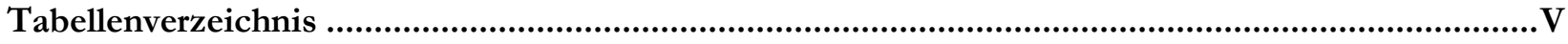

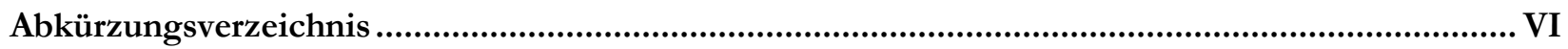

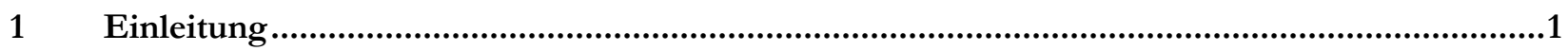

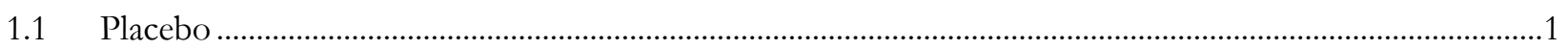

1.1.1 Allgemeine Einführung in die Thematik und der Placeboeffekt ...............................................................1

1.1.2 Die Bedeutung von Placebos für die klinische Forschung ..............................................................................

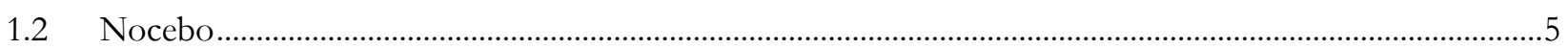

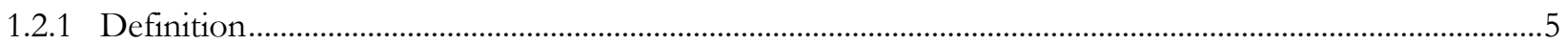

1.2.2 Der Noceboeffekt und seine Relevanz für die klinische Forschung............................................................6

1.3 Die Beeinflussung von Placeboeffekten durch Persönlichkeitsmerkmale ......................................................

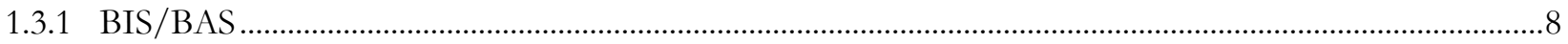

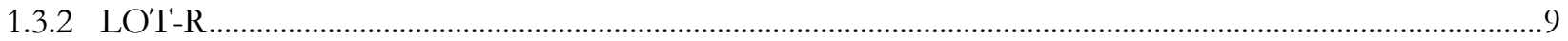

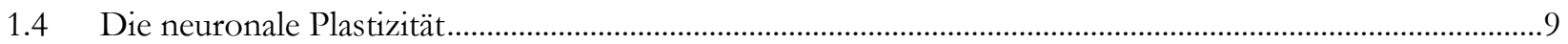

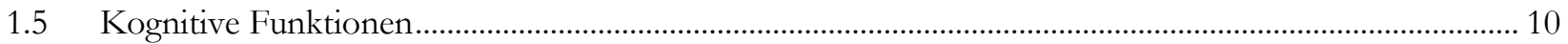

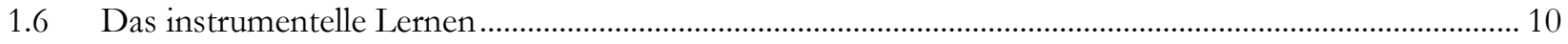

1.7 Transkranielle Gleichstromstimulation...................................................................................................... 12

1.7.1 Überblick zur transkraniellen Gleichstromstimulation................................................................................. 12

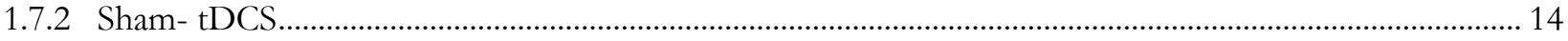

1.7.3 Effekte der tDCS auf die kognitiven Funktionen ...................................................................................... 14

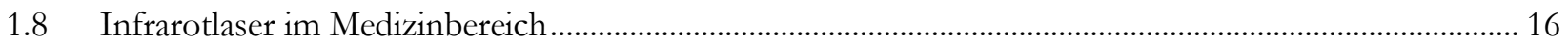

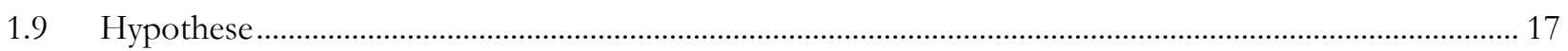

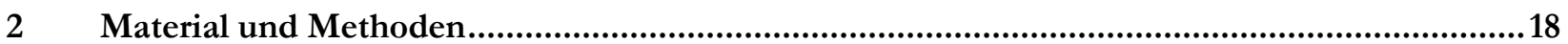

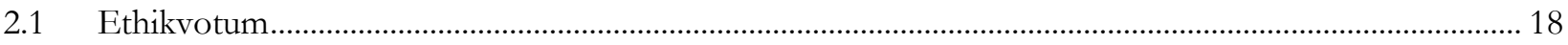

2.2 Probanden und Probandenauswahl....................................................................................................... 18

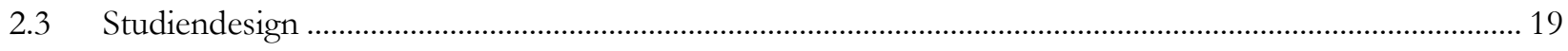

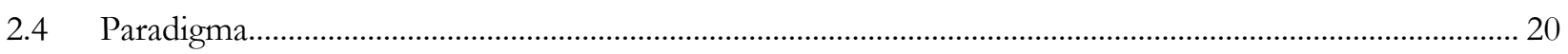

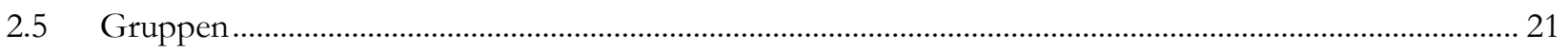

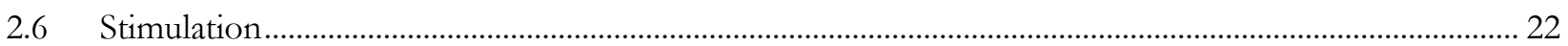




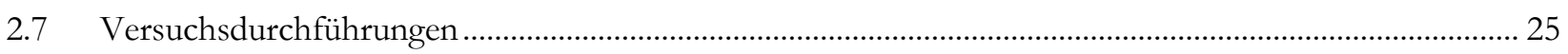

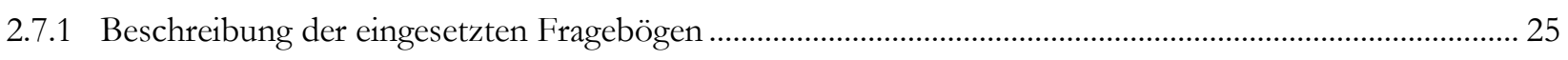

2.7.2 Erste Sitzung - Vorbereitungen.............................................................................................................. 29

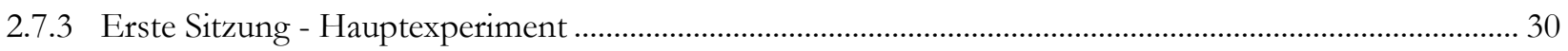

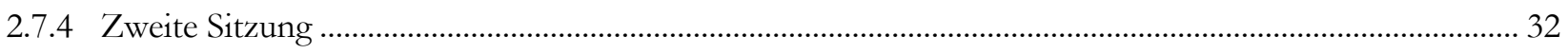

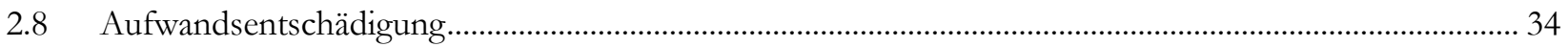

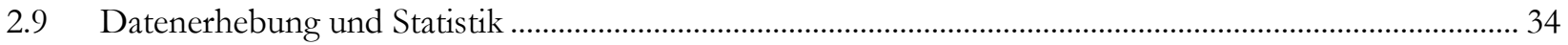

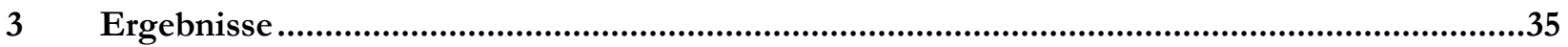

3.1 Stimulationsinduzierte Hautempfindungen............................................................................................ 35

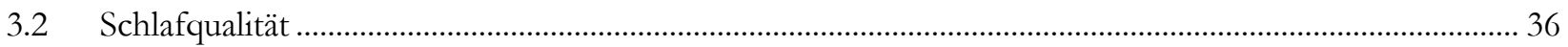

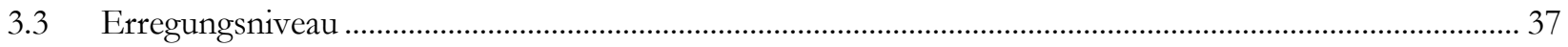

3.4 Der Einfluss von Placebo-und Nocebostimulation auf die kognitive Lernleistung.................................. 38

3.5 Der Zusammenhang zwischen Persönlichkeitseigenschaften und Placebo-/ Noceboeffekten............... 41

3.6 Die Bedeutung der Händigkeit in Bezug auf Placebo-und Noceboeffekte ............................................... 44

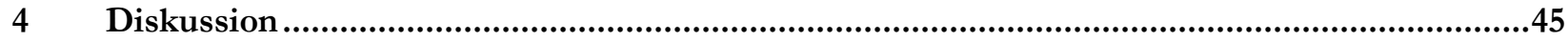

4.1 Experimentelle und klinische Placebostudien zur Beeinflussung von kognitiven Funktionen ................ 46

4.2 Beispiele aus klinischen Placebo-/Nocebostudien........................................................................................... 50

4.3 Erkennung der Placebostimulation ........................................................................................................... 53

4.4 Der Einfluss der Versuchsleiterin auf die Stärke der Placeboeffekte............................................................ 54

4.5 Der Zusammenhang zwischen Persönlichkeitseigenschaften und Placeboeffekten ................................. 56

4.6 Ausblick und klinische Implikationen....................................................................................................... 57

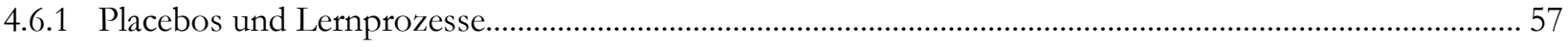

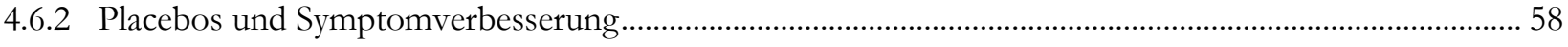

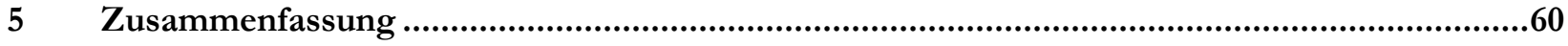

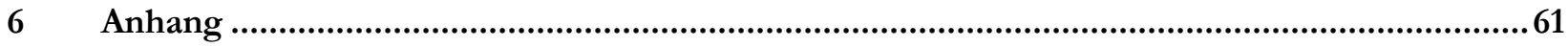

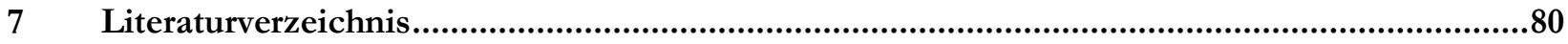




\section{Abbildungsverzeichnis}

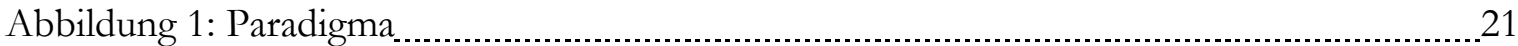

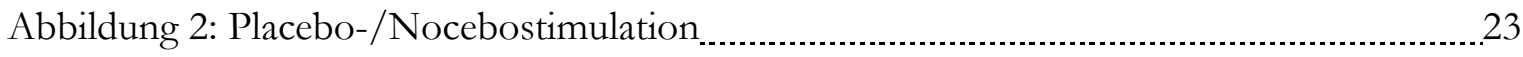

Abbildung 3: Gleichstromstimulator

Abbildung 4: Tastatur zur Aufgabenlösung

Abbildung 5: Placebogruppe: Sitzungsablauf 1. Tag

Abbildung 6: Nocebogruppe: Sitzungsablauf 1. Tag

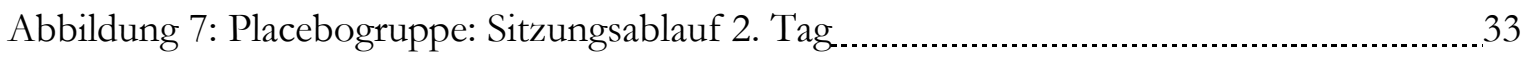

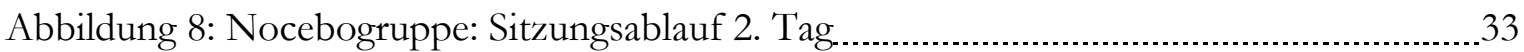




\section{Diagrammverzeichnis}

Diagramm 1: Lernleistung an Tag eins und Tag zwei in beiden Gruppen _...........................41

Diagramm 2: BIS/BAS- Auswertung Placebogruppe............................................................42

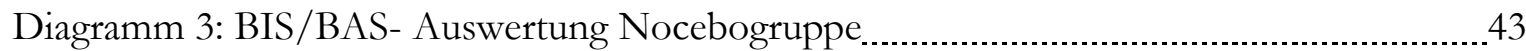




\section{Tabellenverzeichnis}

Tabelle 1: Subjektive kutane Beschwerdeebene................................................................. 36

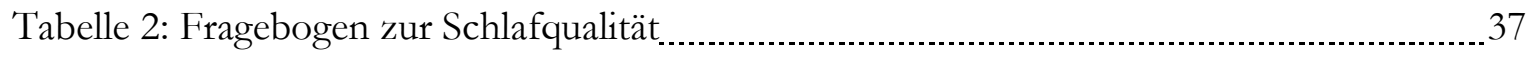

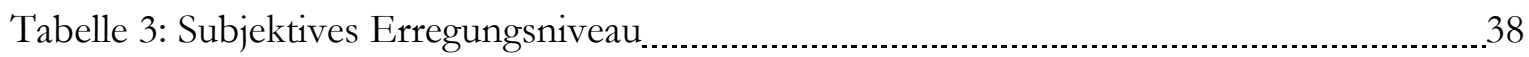

Tabelle 4: Potenzierte Koeffizienten und Vertrauensbereiche am 1. Tag _.............................. 39

Tabelle 5: Potenzierte Koeffizienten und Vertrauensbereiche am 2. Tag _............................... 40 


\section{Abkürzungsverzeichnis}

AC- Stimulation

ARES

BIS/BAS

$\mathrm{Ca}^{2+}$

CBZ

DC- Stimulation

DLPFC

DMO

EEG

EUR

F3

F4

fMRT

FLU

GABA

I/-O- Kurve

LOR

LOT

LOT- R

$\mathrm{mA}$

$\min$

MRT

MT

$\mathrm{Na}^{+}$

NMDA- Rezeptor

NSE

n.s.

p- Wert
Alternating Current Stimulation (Wechselstromstimulation)

Action Regulating Emotion Systems

Behavioral inhibition system, behavioral activation system

(Verhaltenshemmungssystem, Verhaltensaktivierungssystem)

Calcium- Ionen

Carbamazepin

Direct Current Stimulation (Gleichstromstimulation)

Dorsolateraler präfrontaler Kortex

Dextromethorphan

Elektroenzephalogramm

EURO

frontale Elektrodenposition linke Seite (10/ 20 EEG System)

frontale Elektrodenposition rechte Seite (10/ 20 EEG System)

funktionelle Magnetresonanztomographie

Flunarizin

Gamma- Aminobuttersäure

Input/-Output- Kurve

Lorazepam

Life- Orientation- Test

Life- Orientation- Test (revidierte Version)

Milliampere (Einheit für die Stromstärke)

Minute

Magnetresonanztomographie

Motor-Schwellenwert

Natriumion

N- Methyl- D- Aspartat- Rezeptor (Glutamatrezeptor)

neuronenspezifische Enolase (Destruktionsmarker)

nicht signifikant

probability value (Signifikanzwert); Überschreitungswahrscheinlichkeit 


\section{RCT}

$\mathrm{S}$

SD

STN- DBS

tDCS

tES

tRNS
Randomized Controlled Trials

Sekunde

Standard Deviation (Standardabweichung)

subthalamic nucleus deep brain stimulation (tiefe Hirnstimulation)

transcranial Direct Current Stimulation (transkranielle

Gleichstromstimulation)

transcranial Electrical Stimulation (transkranielle elektrische Stimulation)

transcranial Random Noise Stimulation (transkranielle Rauschstromstimulation 


\section{$1 \quad$ Einleitung}

Die immer moderner werdende Welt verlangt von uns, stets „up to date“ zu sein. Dazu ist es essentiell wichtig, sich weiterzubilden und stets Neues hinzuzulernen. In der Schule, im Studium und später natürlich auch im Beruf wächst der Druck, immer auf dem aktuellen Stand zu sein. Doch neben dem täglichen Alltagsstress scheint es manchmal schier unmöglich, neue Informationen schnell aufzunehmen und langfristig im Gehirn abzuspeichern. Wäre es nicht hervorragend, wenn man seine Lernleistung einfach ohne großen Aufwand verbessern könnte?

Es gibt bereits Studien, die sich mit der Thematik Gehirnleistungsverbesserung durch transkranielle Gleichstromstimulation auseinandergesetzt haben (Kincses et al. 2004; Fregni et al. 2005; Boggio et al. 2006; Harty et al. 2014; Zwissler et al. 2014; Cheng et al. 2015; van Steenburgh et al. 2016). Dies ist ein sehr interessanter Ansatz, denn durch das Anlegen eines Gleichstroms, welcher über mindestens zwei Elektroden auf der Kopfhaut appliziert wird, soll es möglich sein, kognitive Lernleistung, das Arbeitsgedächtnis und Aufmerksamkeit zu verbessern.

Doch ist es überhaupt nötig, zur Leistungsverbesserung einen Gleichstrom an unser Gehirn zu leiten? Wie wäre es, wenn allein der Gedanke, dass man einen solchen transkraniellen Gleichstrom appliziert bekommt, bereits zur kognitiven Leistungssteigerung führen würde? Können bereits Gedanken und Erwartungen beziehungsweise eine verbale Manipulation Placeboeffekte auslösen? Können im Gegensatz dazu auch negative Erwartungen beziehungsweise Vorinformationen über eine Hirnstimulation die Gedächtnisleistung verringern? Es ist inzwischen bekannt, dass nicht nur Placebos in Form von Pillen einen Effekt auf den Menschen auslösen können. Selbst Scheinoperationen sollen ihre Wirkung zeigen (Dimond et al. 1960; Moseley et al. 2002).

\subsection{Placebo}

\subsubsection{Allgemeine Einführung in die Thematik und der Placeboeffekt}

Bereits im 18. Jahrhundert wurde der Begriff „Placebo“ in der Medizin eingeführt. „Placebo“ stammt aus dem Lateinischen und wird übersetzt mit ,ich werde gefällig sein“. Im klinischen Wörterbuch Pschyrembel wird „Placebo“ als eine „unwirksame indifferente Substanz“ zur Anwendung „bei Patienten, um einem eingebildeten Bedürfnis nach medikamentöser Therapie zu entsprechen“ und „,im Rahmen der klinischen Erprobung neuer Medikamente (Doppelblindversuch), wenn vorher keine wirksame Therapie bekannt war oder wenn die Wirkung psychischer Einflüsse getestet werden soll“ bezeichnet (Pschyrembel 1994, S. 1205). Placebos können beispielsweise Schmerzen 
oder psychiatrische Symptome lindern und das ohne einen tatsächlichen Wirkstoff zu besitzen. Häufig werden sie auch als inerte Substanzen bezeichnet. Reine Pillenplacebos bestehen aus Stärke, Gelatine, Natriumchlorid, Laktose oder anderen harmlosen Stoffen. Unreine Placebos hingegen können harmlose Substanzen und den tatsächlichen Wirkstoff enthalten. Dieser ist dann jedoch so gering dosiert, dass eine Wirkung als sehr unwahrscheinlich erachtet werden kann (Breidert und Hofbauer 2009).

Als Placeboeffekt bezeichnet man die Wirkung von Placebos auf den Patienten. 1977 spricht Binz von einem „positiven Placeboeffekt“, wenn sich ein Placebo positiv auf den Gesundheitszustand eines Patienten auswirkt (Binz 1977). Diese Bezeichnung kann jedoch inzwischen als veraltet betrachtet werden. Der Begriff „positiver Placeboeffekt“ stammt aus einer Zeit, in der die Thematik „Nocebo“ wissenschaftlich noch kaum Relevanz zeigte. Zum aktuellen Zeitpunkt spricht man bei positiver Beeinflussung des Gesundheitszustandes des Patienten durch ein Scheinmedikament nur noch von „Placeboeffekt“; wirkt sich ein Scheinmedikament negativ aus, verwendet man den Begriff „Noceboeffekt".

Nach Meinung vieler Autoren beruht der Placeboeffekt auf psychischen und mentalen Faktoren, die durch das Gehirn vermittelt werden. Die Placeboforschung konnte in den vergangenen 20 Jahren einen erheblichen Zuwachs im Verständnis der zugrundeliegenden neurobiologischen und psychologischen Mechanismen erlangen (Elsenbruch und Enck 2015). In zahlreichen Untersuchungen kristallisierte sich heraus, dass die Erwartungshaltung und die klassische Konditionierung als zentrale psychologische Erklärungsansätze des Placeboeffektes gelten (Price et al. 1999; Benedetti et al. 2003; Vase et al. 2005; Klinger et al. 2007; Tretrop 2007; Elsenbruch und Enck 2015).

Der Neurotransmitter Dopamin spielt eine entscheidende Rolle bei der Gestaltung von Erwartungen in Bezug auf Belohnungen. Selbst Erwartungen können den Dopaminspiegel beeinflussen. In einer Studie von Schmidt et al. aus dem Jahr 2014 wurde eine Belohnungslernaufgabe von den Patienten ausgeführt. Zeitgleich wurde mit der funktionellen Magnetresonanztomographie (fMRT) die lernbezogene Dopaminfreisetzung im Gehirn sichtbar gemacht. Es konnte beobachtet werden, dass die bloße evozierte Erwartung der Dopaminfreisetzung in Form von manipulierten Probandeninformationen das Belohnungslernen verbessern konnte. Lernbezogene Signale im Striatum und ventromedialen präfrontalen Kortex konnten somit moduliert werden. Diese Ergebnisse deuten auf einen neurobiologischen Mechanismus hin, durch den Erwartungen das Lernen beeinflussen können (Schmidt et al. 2014). Somit gelang es Schmidt et al., anhand eines fMRT die Wirkung der gesteigerten Erwartungshaltung in Form von vermehrter Dopaminausschüttung sichtbar zu 
machen.

Auch das Auftreten des behandelnden Arztes kann Placeboeffekte beeinflussen. Wenn es dem Behandler gelingt, im Patienten Hoffnung und Optimismus durch gute Kommunikation auszulösen, kann der Patient Vertrauen aufbauen und somit Placeboeffekte verstärken (Lang 2006).

Des Weiteren können optimistische Einstellungen des Patienten und des Therapeuten in Bezug auf die Therapie eine erhebliche Verbesserung der Placebowirkung hervorrufen. So kann die Einstellung des Patienten zu dem Medikament das Ergebnis der Behandlung positiv oder negativ beeinflussen (Shapiro und Morris 1978).

Waber et al. konnten in 2008 nachweisen, dass vermeintlich günstige Tabletten einen geringeren Placeboeffekt erzielen als teurere Präparate. Es konnte sogar nachgewiesen werden, dass die Größe, Farbe und Form der eingenommen Pillen, auch wenn sie frei von einem Wirkstoff sind, unterschiedliche Wirkungen auf Patienten auslösen können (Breidert und Hofbauer 2009).

\subsubsection{Die Bedeutung von Placebos für die klinische Forschung}

Placebos werden nicht nur im Rahmen von Therapiemaßnahmen eingesetzt, sondern gelten auch als essentieller Bestandteil von RCT's (Randomized Controlled Trials). Diese Studienart besteht meist aus einer Verumgruppe und einer Placebo- Kontrollgruppe. Dennoch liegt der Fokus der Analyse selten auf der eigentlichen Wirkung des Placebos, sondern viel mehr auf der Überlegenheit des Verumpräparates gegenüber dem Placebo.

Die vollständige Wirkungsweise der Placebos konnte bis heute nicht entschlüsselt werden. Entscheidend ist jedoch vielmehr, dass sie tatsächlich eine Wirkung auslösen. Ab dem 19. Jahrhundert wurden erste Doppelblindstudien in Österreich durchgeführt (Binz 1977). Eine Placebo- Kontrollgruppe ermöglicht aussagekräftige und objektivierbare Ergebnisse. Durch diese kann man die Wirkungen und Nebenwirkungen von neuen Arzneimitteln besser beurteilen. Somit konnte der wissenschaftliche Wert von Arzneimittelstudien durch Placebos deutlich angehoben werden. Wirkt das neu getestete Medikament nicht signifikant besser als das Placebopräparat in der Kontrollgruppe, ist die Chance gering, dass das neue Medikament zugelassen wird. Wenn man bedenkt, dass zahlreiche therapeutische Interventionen ihre Legitimation aus ihrer Überlegenheit gegenüber dem Placebo beziehen, wird die große Bedeutung, die Placebos und Placeboeffekten zukommt, verdeutlicht (Benedetti 2008).

Beecher beschreibt bereits 1955 in dem Werk „The powerful placebo“, dass eine durch- 
schnittliche therapeutische Placebowirkung von 35,2\% \pm 2,2 \% existiert. Diese Ergebnisse erhielt er durch 15 placebokontrollierte Studien mit 1082 Patienten (Beecher 1955).

Studien mit ähnlichen Ergebnissen folgten (Jewett et al. 1990; Clark und Leaverton 1994; Preston et al. 2000; Liccardi et al. 2004; Olshansky 2007). Platsch spricht 2009 in einem Symposiumsvortrag von einer Placebowirkung von 30- 50\% bei oraler Gabe eines Scheinmedikamentes und sogar von 60- 70 \% Erfolgsrate bei invasiveren medizinischen Eingriffen wie Injektionen oder Operationen (Platsch 2009). Kaptchuk et al. konnten in 2006 an Patienten mit chronischen Armschmerzen nachweisen, dass eine Scheinakupunktur einen größeren therapeutischen Effekt, gemessen auf einer visuellen Schmerzskala, auslösen konnte als die Gabe einer Placebopille (Kaptchuk et al. 2006).

Eine Studie von Gliedman et al. beschreibt, dass gerade einmal 25\% der untersuchten Ulkuspatienten auf die Behandlung durch eine Krankenschwester positiv reagierten, wohingegen die Anwesenheit des Arztes sogar bei 70 \% der Patienten eine Verbesserung der Symptome hervorrief. Dabei erhielten alle lediglich ein Placebo. Auch unter optimalen Voraussetzungen können verschiedene Versuchsleiter aufgrund von Vertrauen, Erwartungen und Einstellungen differente Placeboeffekte in Patienten erzielen (Gliedman et al. 1965).

In einer Metaanalyse von Rief et al. wurden 96 placebokontrollierte Studien mit Antidepressiva ausgewertet (Rief et al. 2009a). Man konnte in dieser Arbeit signifikante Placeboeffekte nachweisen. Auffällig war jedoch, dass die Placeboeffektgrößen von den Wissenschaftlern deutlich stärker empfunden wurden als von den Probanden. Des Weiteren konnte nachgewiesen werden, dass die Placeboeffektgrößen mit zunehmenden Publikationsjahr (Zeitraum 1980 bis 2005) um mehr als das Doppelte anstiegen. Dieser Effekt konnte jedoch lediglich bei den Versuchsleitern detektiert werden. Die Selbsteinschätzung der Probanden über die Stärke der Placeboeffekte stieg hingegen nicht mit steigendem Publikationsjahr. Diese Erkenntnisse deuten auf einen starken Einfluss der Erwartungen der Versuchsleiter im Zusammenhang mit Placeboeffekten hin (Rief et al. 2009a). Häuser et al. konnten bei Patienten mit Fibromyalgiesyndrom nachweisen, dass die Überlegenheit der medikamentösen Behandlung lediglich gering gegenüber dem Placebo war (Häuser et al. 2012a).

Zusammenfassend ist es wichtig festzuhalten, dass Placeboeffekte in RCT'S zu kontrollieren und zu minimieren sind, während man sie im Praxisalltag eher stärken sollte, damit Patienten davon profitieren können (Benedetti et al. 2006; Enck et al. 2013; Häuser et al. 2012a). 


\subsection{Nocebo}

\subsubsection{Definition}

Im Jahr 1961 prägte Walter P. Kennedy diesen Begriff (Kennedy 1961). Erst in den letzten Jahren ist ein Zuwachs im wissenschaftlichen Interesse zu dieser Thematik zu verzeichnen. Trotzdem ist der Placeboeffekt deutlich bekannter als der Noceboeffekt.

Der Begriff „Nocebo” bedeutet übersetzt „ich werde schaden” und steht für das Gegenteil von Placebo. Er bezeichnet eine negative Reaktion auf ein Medikament, welches in Wirklichkeit frei von einem Wirkstoff ist. Noceboeffekte beruhen analog den Placeboeffekten auf einer bestimmten Erwartungshaltung. Auch Fehldiagnosen von Ärzten oder ausführliche Erläuterungen zu eventuellen Nebenwirkungen, zum Beispiel in Beipackzetteln von Medikamenten oder Probandeninformationen zu einer Studie können im Patienten eine negative Erwartungshaltung auslösen und somit Krankheitsbilder hervorrufen, für die es keine weitere medizinische Ursache gibt (Wells und Kaptchuk 2012).

Wenn ein Behandler den Patienten im Aufklärungsgespräch auf mögliche zu erwartende Nebenwirkungen hinweist, ist die Gefahr, dass der Patient durch eine negative Erwartungshaltung einen Noceboeffekt entwickelt, relativ hoch. Wenn der Arzt jedoch während des Gespräches den Fokus der Erklärungen auf die positiven Wirkungen des Medikamentes legt und gleichzeitig erklärt, dass die folgende Behandlung in der Regel gut verträglich ist, können Noceboeffekte verringert werden (Häuser et al. 2012b).

Wie entscheidend eine Patienten- bzw. Probandenvorinformation in Bezug auf erwartungsinduzierte Noceboeffekte sein kann, soll folgendes Beispiel verdeutlichen. In einer randomisierten Studie mit männlichen Hypertonie- Patienten wurden 3 Teilnehmergruppen gebildet. Alle Teilnehmer erhielten Metoprolol, einen Betablocker. Gruppe 1 wurde vorab über die mögliche unerwünschte Nebenwirkung der erektilen Dysfunktion aufgeklärt. Gruppe 2 erhielt keine Vorinformationen über diese eventuell auftretende Nebenwirkung. Die Teilnehmer erfuhren lediglich, dass sie mit Metoprolol behandelt werden. Die Teilnehmer der Gruppe 3 erhielten weder den Medikamentennamen noch die Information über dessen mögliche unerwünschte Nebenwirkung der erektilen Dysfunktion. In Gruppe 1 berichteten 32\% über die Nebenwirkung der erektilen Dysfunktion. In Gruppe 2 lag die Nebenwirkungsrate bei 13\%. In der dritten Gruppe klagten lediglich 8\% über eine erektile Dysfunktion (Cocco 2009).

Colloca und Benedetti konnten nachweisen, dass inerte Substanzen eine Nocebo- Hyperalge- 
sie provozieren können. Negative Erwartungen an eine verabreichte inerte Substanz können im Patienten antizipatorische Ängste auslösen. Diese Ängste lösen eine Aktivierung des Botenstoffes Cholecystokinin aus, welcher wiederum die Schmerzübertragung erleichtert. Somit kann allein der Gedanke, dass eine verabreichte schmerzverstärkende Substanz, die in Wirklichkeit frei von einem Wirkstoff ist, so intensiv sein, dass durch ausgelöste Ängste tatsächlich Schmerzen verstärkt werden (Colloca und Benedetti 2007).

\subsubsection{Der Noceboeffekt und seine Relevanz für die klinische Forschung}

In placebokontrollierten Doppelblindstudien zur Neuzulassung von Medikamenten lassen sich häufig Noceboeffekte beobachten. In diesem Fall werden alle Patienten, unabhängig davon, ob sie ein Scheinmedikament oder den tatsächlichen Wirkstoff erhalten, über mögliche zu erwartende Nebenwirkungen im Vorfeld aufgeklärt. Bei etwa 25\% der Mitglieder der Placebogruppe tritt eine der genannten Nebenwirkungen auf (Clark und Leaverton 1994). Ein Beispiel ist das Auftreten einer Hypervagotonie, welche sich in einer Doppelblindstudie eines Calciumantagonisten bei den Probanden durch Herzrhythmusstörungen manifestierte, obwohl diese nur das Placebo erhalten hatten (Cutler et al. 1991). Der Noceboeffekt kann sich durch subjektive Symptome wie Übelkeit oder Kopfschmerzen kennzeichnen. Es sind allerdings teilweise auch objektive Symptome wie erhöhter Blutdruck und Puls diagnostizierbar.

Eine Metaanalyse mit Daten aus 143 placebokontrollierten RCT's kam zu dem Ergebnis, dass Studien mit trizyklischen Antidepressiva deutlich mehr Nebenwirkungen in der Placebogruppe zeigten als Studien mit Serotonin- Wiederaufnahme- Hemmern. Als Nebenwirkungen nach Placebogabe wurden beispielsweise ein trockener Mund, Müdigkeit, sexuelle Dysfunktionen sowie Obstipation erfasst. Die erfassten Nebenwirkungen waren sowohl in der Placebogruppe als auch in der Verumgruppe nachweisbar. Es konnte nachgewiesen werden, dass die empfundenen Nebenwirkungen mit den Erwartungen der Probanden, aber auch der Versuchsleiter verknüpft sind (Rief et al. 2009b).

Anhand von weiteren Metaanalysen konnte inzwischen bestätigt werden, dass nicht nur die Erwartungen des Probanden, sondern auch die Erwartungshaltung des Arztes bzw. des Experimentators unerwünschte Arzneimittelnebenwirkungen in placebokontrollierten Studien auslösen können (Mora et al. 2011). Außerdem gibt es eine Altersabhängigkeit, z.B. Rosenzeig et al. 1993 und Ströhle 2000 kamen zu dem Ergebnis, dass besonders ältere Menschen und Frauen zum Aufbau von Noceboeffekten neigen. 
Petersen et al. untersuchten in einer Metaanalyse zahlreiche Studien zur Thematik Noceboeffekte und Schmerzen. In Studien, in denen Noceboeffekte durch eine Kombination von verbaler Suggestion und Konditionierung induziert wurden, war die Effektgröße deutlich höher als in Studien, in denen Noceboeffekte allein durch verbale Suggestion ausgelöst wurden. Diese Erkenntnisse ähneln denen der Placeboliteratur. Schlussfolgernd sollten Noceboeffekte in der klinischen Praxis minimiert werden (Petersen et al. 2014).

\subsection{Die Beeinflussung von Placeboeffekten durch Persönlichkeitsmerkmale}

Der Placeboeffekt konnte bis zum heutigen Zeitpunkt noch nicht vollständig durchleuchtet und erklärt werden. Es stellt sich immer wieder die Frage, warum manche Menschen besonders empfänglich, andere hingegen eher unempfänglich für die Wirkung von Placebos sind. Ein Ansatz könnte in den Persönlichkeitsmerkmalen der Probanden und Patienten liegen.

Es wurden in der Vergangenheit erst wenige Studien durchgeführt, die einen Zusammenhang zwischen Persönlichkeitsmerkmalen und der Stärke von Placeboeffekten untersuchten. Eine Studie kam zu dem Ergebnis, dass besonders direkte und stressresistente Probanden auf die Placebos reagierten. Des Weiteren sollen ausgeglichene, aufrichtige und altruistische Menschen stärkere Placeboeffekte in Bezug auf Schmerzlinderung zeigen. Jene Probanden, die sich laut Persönlichkeitstest schnell verärgern lassen, zeigen keine ausreichenden Effekte auf eine Behandlung durch Placebos (Pecina et al. 2013). Auch abenteuerlustige Menschen sollen besser auf eine Scheinbehandlung reagieren als zurückhaltende Menschen (Schweinhardt et al. 2009).

Horing et al. 2014 überprüften in einem Review Publikationen zur Thematik Placebobebandlungen mit der Zielsetzung, Artikel zu identifizieren, die qualitativ hochwertige Daten zur „vorhersehbaren Placeboantwort“ liefern. Trotz der Heterogenität der Ergebnisse konnte eine gewisse Kongruenz von Prädiktoren nachgewiesen werden. Hierzu zählen Optimismus, Abenteuerlust und Selbstbewusstsein, jedoch auch Ängstlichkeit.

Entsprechende Persönlichkeitsmerkmale lassen sich anhand von definierten psychologischen Tests nachweisen. Hierzu eignen sich besonders die BIS/ BAS Skala und der LOT-R- Test. Eine deutsche Version dieser Fragebögen, die jeder Proband vor unserem Experiment ausfüllt, ist im Anhang dieser Arbeit zu finden (Anlage 3 und 6). 


\subsubsection{BIS/BAS}

J. A. Gray postulierte eine Theorie zum Verhaltenshemmsystem (Behavioral Inhibition System, BIS) und zum Verhaltensaktivierungssystem (Behavioral Approach System, BAS). Dieses System ist von besonderer Relevanz für die biopsychologisch orientierte Persönlichkeitsforschung. Das menschliche Verhalten soll demnach durch zwei allgemeine Motivationssysteme (Verhaltenshemmsystem und Verhaltensaktivierungssystem) beeinflusst werden können. BIS ist empfänglich für Signale von Neuheit, Nichtbelohnung und Bestrafung. Eine Aktivierung des BIS löst eine Hemmung der Bewegung auf ein Ziel hin aus. Eine höhere BIS- Sensitivität wird mit Furcht, Ängstlichkeit, Frustration und Traurigkeit assoziiert. Das BAS hingegen ist empfänglich für Signale der Belohnung, Nichtbestrafung und Flucht vor Bestrafung. Eine erhöhte BAS- Sensitivität wird somit in einen Zusammenhang mit positiven Gefühlen wie Freude und Hoffnung gebracht. Zielgerichtete Bemühungen zum Erleben positiver Gefühle werden assoziiert.

Zur Erfassung der entsprechenden Persönlichkeitsmerkmale liegt der BIS/BAS-Fragebogen von Carver und White vor, der vier Skalen (BIS, BAS Fun Seeking, BAS Drive und BAS Reward Responsiveness) umfasst. Die Erfassung im Bereich BAS erfolgt über Aussagen zu Vergnügungssuche, Antrieb und Belohnungssensitivität. Die Erfassung von BIS erfolgt über Aussagen zur Verhaltenshemmung.

Die Skalenkonstuktionen von Carver und White zur Erfassung der BIS- und BAS-Sensitivität stellen zwar eine Möglichkeit der Bestimmung psychometrischer Eigenschaften dar, jedoch konnten diese hinsichtlich der Skaleneigenschaften noch nicht als völlig zufrieden stellend bewertet werden. Aufbauend auf dem Grayschen Modell und in Anbetracht der zentralen Überlegungen von Carver und White wurden deutsche Skalen zur Erfassung der Sensitivität der beiden Emotionssysteme (Action Regulating Emotion Systems; ARES) von Strobel et al. 2001 neu konstruiert (Strobel et al. 2001). Hierbei gibt es eine Langfassung mit 58 Items und eine Kurzfassung, bestehend aus 20 Items. Die ARES-Skalen enthalten zur spezifischeren Bestimmung die Subskalen BIS I “Ängstlichkeit” und BIS II "Frustration” zur Erfassung der individuellen BIS- Sensitivität. Außerdem sind die Subskalen BAS I “Antrieb” und BAS II "Freude” zur Erfassung der individuellen BAS-Sensitivität vorgesehen (Hartig und Moosbrugger 2003). 


\subsubsection{LOT-R}

Die LOT-R Skala ist ein sehr häufig eingesetztes Verfahren in der Optimismusforschung und dient somit ebenfalls der Detektion von Persönlichkeitsmerkmalen. Zur Erfassung des dispositionellen Optimismus wurde von Scheier und Carver 1985 der Life-Orientation-Test (LOT) konstruiert. Dieser wurde auch in einer deutschen Übersetzung von Wieland-Eckelmann und Carver im Jahr 1990 veröffentlicht. Ungeachtet der Popularität des LOT gab es wesentliche Kritikpunkte an diesem Instrument. In Reaktion auf einige Kritikpunkte wurde der LOT 1994 überarbeitet und in einer revidierten Version (LOT-R) von Scheier, Carver und Bridges 1994 veröffentlicht. Die Korrelation zwischen beiden Skalen beträgt $\mathrm{r}=0.95$, so dass nach Meinung der Autoren die Äquivalenz der Verfahren gegeben ist. In der revidierten Version wurden zwei Items der ursprünglichen LOT-Skala eliminiert (Glaesmer et al. 2008).

\subsection{Die neuronale Plastizität}

Unter dem Begriff der neuronalen Plastizität kann man die Veränderbarkeit neuronaler Verbindungen im Nervensystem verstehen (Kapfhammer 2000). Neuronale Verbindungen sind nicht starr und invariabel, sondern können aufgrund bestimmter funktioneller Geschehen, z.B. bei Lernprozessen oder nach dem Verlust von Nervenzellen oder Axonen, Veränderungen unterliegen. Es lässt sich u.a. eine funktionelle und strukturelle Plastizität unterscheiden.

\section{Funktionelle Plastizität}

Hierbei verändert sich insbesondere die Effizienz einer synaptischen Übertragung. Ohne Änderung der anatomischen Verbindungsstruktur können dennoch Änderungen in der funktionellen Verschaltung des Netzwerkes im Gehirn vorgenommen werden. Die funktionelle Plastizität ist für Lernprozesse von besonderer Bedeutung, da auf diese Art die synaptische Übertragung in mehreren, gleichzeitig aktiven Synapsen verstärkt werden kann. Für diese Verstärkung hat an vielen Synapsen die Aktivität des NMDA-Rezeptors (Glutamatrezeptor) eine wichtige Funktion. Dieser wird erst bei einer Depolarisation der Zelle aktiviert und kann über einen vermehrten $\mathrm{Ca}^{2+}$-Ionen-Einstrom langfristige Veränderungen der synaptischen Effizienz auslösen (Kapfhammer 2000). 


\section{Strukturelle Plastizität}

Die strukturelle Plastizität kann auch als anatomische Plastizität bezeichnet werden. Hierbei kommt es im Gegensatz zur funktionellen Plastizität zu anatomischen Veränderungen der Hirnstruktur. Es können beispielsweise neue Synapsen oder sogar neue Axone gebildet werden (Kapfhammer 2000). Ein Grund für die Neubildung könnte eine Denervation eines Zielgebietes sein. Die noch intakt gebliebenen Axone in diesem Bereich können neue Kollaterale bilden und somit die Neurone re-inervieren. Dieser Vorgang kann auch durch Läsionen im adulten Nervensystem ausgelöst werden. Im Gehirn eines jungen Menschen können solche Regenerationsvorgänge besser ablaufen als bei älteren Menschen. Das juvenile, unreife Gehirn zeigt eine hohe Veränderbarkeit aufgrund verschiedener sensorischer Erfahrungen (Kapfhammer 2000).

\subsection{Kognitive Funktionen}

Der Begriff Kognition stammt vom lateinischen Wort „cognoscere“ ab und bedeutet sinngemäß erkennen, erfahren oder kennenlernen. Unter „Kognition“ versteht man häufig das Denken im umfassenden Sinne. Kognitive Fähigkeiten in uns Menschen umfassen beispielsweise Problemlösungen, Erwartungen, Konzentration, Aufmerksamkeit und Wahrnehmung, Lernen, Erinnerungen, Wissen, Kreativität und Prozesse der Informationsverarbeitung. Informationen, die wir im Langzeitgedächtnis speichern, können jedoch häufig durch positive oder negative Erwartungshaltung beeinflusst werden. Alle diese Fähigkeiten können durch gezieltes Hirntraining gesteigert werden. Intensive Lernprozesse können Veränderungen in der funktionellen Verschaltung des Netzwerkes im Gehirn auslösen und somit Einfluss auf die neuronale Plastizität nehmen. Eine passende Definition haben Kaminski und Neisser formuliert: „Sammelname für alle Vorgänge oder Strukturen, die mit dem Gewahrwerden und Erkennen zusammenhängen, wie Wahrnehmung, Erinnerung (Wiedererkennen), Vorstellung, Begriff, Gedanke, aber auch Vermutung, Erwartung, Plan und Problemlösen“ (Kaminski und Neisser 1994, S. 387).

\subsection{Das instrumentelle Lernen}

Das instrumentelle Lernen, auch instrumentale Konditionierung genannt, oder umgangssprachlich „Lernen durch Belohnung/Bestrafung“, beschreibt, dass das persönliche Verhalten eines Lebewesens ein Instrument ist, welches eine bestimmte Konsequenz hervorrufen kann. Es kann auch die passende Bezeichnung „Lernen am Erfolg“ benutzt werden. Einem positiven Verhalten 
folgen positive Konsequenzen, einem negativen Verhalten entsprechend negative Konsequenzen. Mit anderen Worten: der Mensch versucht durch eine bestimmte Verhaltensweise eine entsprechende Konsequenz zu erhalten. Somit kann das eigene Verhalten als Instrument zur Erlangung eines Zieles eingesetzt werden. Durch häufig wiederholte Konsequenzen kann im Umkehrschluss auch ein bestimmtes Verhalten erlernt werden.

Im Jahr 1898 wurde die instrumentale Konditionierung durch Thorndikes Experimente im Rahmen seiner Dissertation erforscht. Hierbei hatten hungrige Katzen die Aufgabe, sich aus einem Käfig zu befreien, um somit das Futter zu erreichen. Die nervösen Tiere, die sich jedoch vornehmlich aus ihrer Gefangenschaft befreien wollten, betätigten irgendwann zufällig einen richten Hebel und konnten sich somit aus dem Käfig befreien. Bei jedem erneuten Versuch gelang es den Katzen immer schneller, den richtigen Hebel zu bedienen und nach einigen Versuchswiederholungen betätigten die Katzen den Hebel sofort nachdem sie in den Käfig gesperrt wurden. Wenn nun die bestimmte Verhaltensweise, in diesem Fall die Betätigung des Hebels zur erwünschten Folge, also der Befreiung der Katze aus dem Käfig führte, so erhöhte sich die Wahrscheinlichkeit, dass die Katze das entsprechende Verhalten zukünftig wieder zeigt. Somit lernten die Katzen, dass bestimmte Verhaltensmuster bestimmte Konsequenzen auslösen (Edelmann 2000).

Die instrumentelle Konditionierung unterscheidet sich von der klassischen Konditionierung. Die klassische Konditionierung hingegen ist eine Form des Lernens, in der ein Reiz ein bevorstehendes Ereignis ankündigt und somit eine Reaktion ausgelöst wird. Ein bekanntes Beispiel für die klassische Konditionierung stellen die Speichelflussexperimente von Ivan Pavlov aus dem Jahr 1918 dar. Hierbei wurde ein Hund stets nach einem Glockenton gefüttert. Futter löst im Hund Speichelfluss aus. Nach einigen Versuchen reichte allein das Ertönen der Glocke aus, um den Speichelfluss des Hundes anzuregen. Somit wurde der Hund auf einen entsprechenden Reiz, in diesem Fall den Glockenton, konditioniert. Pavlovs Hunde haben gelernt, dass nach einem bestimmten Reiz ein bestimmtes Ereignis eintritt. Thorndikes Katzen haben dagegen gelernt, dass ein bestimmtes Verhalten eine bestimmte Konsequenz zur Folge hat.

Gegenwärtig gibt es zahlreiche Studien, die versuchen, eine Gehirnleistungsverbesserung durch Gehirnstimulation, beispielsweise in Form von tDCS, zu evozieren. Eine solche Gehirnleistungsverbesserung könnte zum Beispiel eine gesteigerte Leistungsfähigkeit beim Lösen von Aufgaben und Problemfragestellungen nach sich ziehen. 


\subsection{Transkranielle Gleichstromstimulation}

\subsection{1 Überblick zur transkraniellen Gleichstromstimulation}

Der Begriff transkranielle Gleichstromstimulation, (transcranial direct current stimulation tDCS), steht für eine nicht-invasive, schmerzfreie Methode zur Hirnstimulation, mit der wahrscheinlich eine Modulation der Exzitabilität des menschlichen Kortex möglich ist. Diese Veränderung der kortikalen Exzitabilität ist stets reversibel. Wichtige Parameter stellen die Polarität der Stimulation (anodal oder kathodal), der Stimulationsort, die Dauer der Stimulation sowie die Stimulationsintensität dar. Während einer solchen Stimulation wird Gleichstrom mit einer Stromstärke von 1-2 mA tonisch appliziert. Dieser wird über mindestens zwei auf der Kopfhaut liegende Elektroden verabreicht und fließt zwischen den Elektroden durch den Kortex und führt dort zu einer Verschiebung des Ruhepotentials der Neurone, ohne dabei selbst Aktionspotentiale auszulösen (Nitsche und Paulus 2007).

Während einer anodalen Stimulation kann eine Erregungssteigerung von kortikalen Neuronen wahrscheinlich durch Depolarisierung erreicht werden. Eine kathodale Stimulation hingegen führt zu einer Inhibition der Neurone durch Hyperpolarisation. Die Dauer der Stimulation spielt eine entscheidende Rolle in der Wirkung auf den Kortex. Bei sehr kurzen Stimulationszeiten (unter 3 Minuten) hält die Erregungsänderung im Kortex nur während der Zeit der Stimulation an (Nitsche et al. 2008). Ab einer Stimulationsdauer von 9-13 min lassen sich jedoch auch noch nach Stimulationsende Effekte auf den motorischen Kortex nachweisen. Nitsche und Paulus bezeichneten dieses Phänomen als ,after-effects' (Nitsche und Paulus 2000). Multiple Sitzungen mit tDCS in definierten Abständen sollen sogar Effekte in dem Gehirn auslösen, die mehrere Wochen anhalten (Reis et al. 2009). Jedoch konnten die Mechanismen, die diesen Veränderungen zugrunde liegen, noch nicht vollständig erklärt werden. Die bereits veröffentlichten Ergebnisse wurden anhand zahlreicher Versuche mit neuromodulatorisch wirksamen Medikamenten ermittelt. Der Calciumkanalblocker Flunarizin (FLU) reduziert die Wirkung der tDCS und der Natriumkanalblocker Carbamazepin (CBZ) verhindert eine Entwicklung von Effekten der anodalen Stimulation (Nitsche et al. 2003b). Weder Dextromethorphan (DMO), ein NMDA Rezeptorantagonist (Nitsche et al. 2003b) noch Lorazepam (LOR), ein GABAA Rezeptoragonist (Nitsche et al. 2004) zeigten einen modulatorischen Effekt auf die stimuläre Antwort. Im Gegensatz zur anodischen Stimulation hatte weder die Blockade von spannungsabhängigen $\mathrm{Ca}^{2+}$ noch der $\mathrm{Na}^{+}$Kanäle Auswirkungen auf die Erregbarkeitsverschiebungen (Nitsche et al. 2003b). 
der Modulation der GABAergen als auch der glutamatergen Synapsen abhängig zu sein scheint. Die Nachwirkungen werden durch Acetylcholin und Serotonin moduliert. Es ist nicht genau bekannt, in welchem Ausmaß die Nachwirkungen von kathodischer tDCS abhängig von Membranpotentialänderungen sind (Nitsche et al. 2003b). Die Nachwirkungen der kathodischen tDCS sind jedoch von der Modulation der glutamatergen Synapsen abhängig. Hierbei handelt es sich wahrscheinlich um die intrakortikalen Interneurone. Die Nachwirkungen der kathodalen tDCS werden durch Dopamin, Acetylcholin und Serotonin moduliert (Stagg und Nitsche 2011).

Einige Studien berichten über die Sicherheit der tDCS. Bei zehn gesunden Probanden konnten Nitsche et al. sowohl 30 als auch 60 Minuten nach tDCS im MRT weder Ödeme noch Veränderungen des Hirngewebes oder der Blut- Hirn- Schranke erkennen. Des Weiteren war nach tDCS der neuronale Destruktionsmarker ,neuronenspezifische Enolase (NSE)“ nicht erhöht. Untersuchungen mittels MRT und EEG zeigten keine pathologischen Veränderungen als Folge der Behandlung (Nitsche et al. 2003a). Poreisz et al. untersuchten in einer Studie ebenfalls Nebenwirkungen nach transkranieller Gleichstromstimulation. Im Gegensatz zu Nitsche et al. 2003a bestand das Probandenkollektiv aus gesunden Teilnehmern sowie Migräne-, Schlaganfall- und Tinnitus Patienten. Folgende Nebenwirkungen wurden untersucht: Kopfschmerzen, Konzentrationsstörungen, Sehstörungen und unangenehme Hautempfindungen unter den Elektroden. 70,6\% der Probanden beschrieben ein leichtes Kribbeln und 30,4\% ein leichtes Brennen unter den Elektroden. Die gesunden Probanden empfanden das Kribbeln signifikant stärker als die Patienten. Kopfschmerzen traten bei 11,8\% der Teilnehmer auf. Kopfschmerzen traten jedoch signifikant häufiger bei den Patienten im Gegensatz zu den gesunden Probanden auf (Poreisz et al. 2007).

Es handelt sich somit bei der transkraniellen Gleichstromstimulation um eine nicht- invasive, im Allgemeinen gut verträgliche, stets reversible Methode, mit der es möglich ist, Einfluss auf die kortikale Exzitabilität zu nehmen. Dieses Verfahren wurde mit seinen umfassenden Einsatzmöglichkeiten bereits in zahlreichen Studien erforscht. Es findet beispielsweise Einsatz in der Behandlung von Schmerzen, Epilepsie, Zustand nach Apoplex und Depressionen (Stagg und Nitsche 2011).

In unserem Experiment diente die tDCS in Form einer Sham-tDCS dem Aufbau einer Erwartungshaltung in den Probanden. Die tDCS sollte in diesem Fall laut Probandeninformation zu einer Verbesserung beziehungsweise Verschlechterung der kognitiven Funktionen führen. 


\subsubsection{Sham- tDCS}

Die transkranielle Gleichstromstimulation eignet sich besonders gut für eine ShamStimulation, da der Proband über einen kurzen Zeitabschnitt einen leicht spürbaren Strom über die Kopfhaut appliziert bekommen kann, ohne dass es dabei zu einer Veränderung der kortikalen Erregbarkeit kommt (Nitsche und Paulus 2007; Ambrus et al. 2012). Somit nimmt der Proband eine Stimulation wahr, ohne zu vermuten, dass es sich hierbei um eine reine Placebostimulation handeln könnte. Diese Eigenschaft machen sich häufig doppelverblindete, randomisierte Studien zum Vorteil, um optimale Bedingungen für die Placebokontrollgruppe zu schaffen. In unserem Experiment wurde ein definiertes Stimulationsprotokoll verwendet. Die einminütige Sham-Stimulation gliederte sich in 20s ,ramp up', 30s $1 \mathrm{~mA}$ Stimulation und 10s ,ramp down'. Dies bedeutet, dass sich für 20s ein Gleichstrom aufbaut, dieser 30s konstant gehalten wird und in den nächsten 10s wieder abgebaut wird.

In einer Studie aus dem Jahr 2006 sollten sowohl gesunde Probanden als auch SchlaganfallPatienten Schmerzempfindungen und Ermüdung während der Stimulation mittels einer visuellen Analogskala klassifizieren. Hierbei konnten Sham- und Verumstimulation nicht unterschieden werden. Es wurde in diesem Fall eine 20 minütige Stimulation verwendet (Gandiga et al. 2006).

Wir verwenden jedoch bewusst eine sehr kurze Stimulationsdauer von gerade einmal einer Minute um definitiv ausschließen zu können, dass es durch die tDCS nicht zu eventuellen Veränderungen der kognitiven Fähigkeiten als Folge einer Erregungsänderung im Kortex kommen kann. Außerdem sollte eine Stimulation von einer Minute eine Neuerung im Gegensatz zu bisher durchgeführten tDCS-Studien darstellen. Somit wird sowohl den Probanden als auch der Versuchsleiterin suggeriert, dass das Experiment eine Differenz zu bereits bekannten Studien darstellt und somit von hohem wissenschaftlichem Interesse zu sein scheint. Hiermit wird der Verdacht, dass es sich bei dem Experiment um eine Placebostudie handeln könnte, umgangen.

\subsubsection{Effekte der tDCS auf die kognitiven Funktionen}

Es konnte belegt werden, dass tDCS sowohl im motorischen als auch im visuellen, präfrontalen und somatosensorischen Kortex Effekte auslösen kann. TDCS eignet sich nicht nur zur Erzeugung, sondern auch zur Modulation von neuroplastischen Vorgängen. Erste Studien belegten die Effektivität der Stimulation zur Modulation übungsabhängiger Neuroplastizität, visuomotorischer Koordination sowie der Modulation von motorischen und semantischen Lernvorgängen (Nitsche et 
al. 2004).

Zum Nachweis der Wirkung der tDCS auf die Leistung des Arbeitsgedächtnisses werden hauptsächlich präfrontale Regionen stimuliert, weil diese die Referenzregionen der Kognition sind und bei psychiatrischen Störungen eine entscheidende Rolle spielen können. Bei anodaler tDCS des linken präfrontalen Kortex ergab eine Studie an gesunden Probanden Leistungsverbesserungen im impliziten Lernen (Kincses et al. 2004). Auch Fregni und Mitarbeiter konnten nach Stimulation des linken präfrontalen Kortex mittels anodaler tDCS eine Verbesserung des Arbeitsgedächtnisses feststellen. Die gesunden Probanden wurden wie folgt untersucht: auf einem Bildschirm erschien alle zwei Sekunden ein neuer Buchstabe für 30 Millisekunden. Der Versuchsteilnehmer sollte immer dann auf einen Knopf drücken, wenn ein Buchstabe dem drittletzten aus der Reihe entsprach. Während die anodale Stimulation zu einer Verbesserung des Arbeitsgedächtnisses führte, erbrachten kathodale -sowie Placebostimulation keine Änderungen (Fregni et al. 2005).

Boggio hingegen untersuchte das Arbeitsgedächtnis bei 18 Parkinsonpatienten vor und nach anodaler Stimulation mit $1 \mathrm{~mA}$ und $2 \mathrm{~mA}$ über dem linken dorsolateralen präfrontalen Kortex (DLPFC) und dem Motorkortex. Als Ergebnis zeigte sich ein verbessertes Arbeitsgedächtnis nach anodaler tDCS mit einer Stimulationsintensität von $2 \mathrm{~mA}$ über dem linken DLPFC (Boggio et al. 2006). Weitere Effekte auf kognitive Funktionen durch bilaterale Stimulation des DLPFC konnten Fecteau et al. zeigen. Die Anode wurde über den rechten oder den linken DLPFC gesetzt und die Kathode über den kontralateralen DLPFC. Dadurch konnte das Risiko-Verhalten in unklaren Entscheidungssituationen verringert werden (Fecteau et al. 2007).

Im Gegensatz zu den hier erwähnten Studien konnten Marshall et al. eine Verschlechterung des Arbeitsgedächtnisses während bifrontaler anodaler wie auch kathodaler tDCS nachweisen (Marshall et al. 2005).

Eine aktuelle Studie aus dem Jahr 2015 widerspricht jedoch den Ergebnissen aller vorherigen Studien. In diesem Review wird behauptet, dass eine einmalige tDCS keinen signifikanten Einfluss auf die kognitiven Funktionen haben kann. Zur Ergebnisfindung wurden Daten aus 59 Analysen mit der entsprechenden Thematik gesammelt und ausgewertet (Horvath et al. 2015).

Es ist dennoch erkennbar, dass anodale tDCS durch neuromodulatorische Vorgänge im Gehirn Verbesserungen der kognitiven Funktionen evozieren kann. Dieser Effekt kann beispielsweise für Parkinsonpatienten, Alzheimerpatienten sowie Schlaganfallpatienten und Menschen, die unter Depressionen leiden, einen therapeutischen Benefit bewirken. 


\subsection{Infrarotlaser im Medizinbereich}

Infrarotlaser, die im medizinischen Bereich Anwendung finden, arbeiten mit kurzen Hitzeimpulsen. Mit ihnen kann beispielsweise die Funktion von Nervenfasern untersucht werden. Diese Reize können vom Menschen weder visuell noch akustisch wahrgenommen werden. Guosong Hong und seinen Kollegen von der Stanford University gelang es mit Hilfe eines Infrarotlasers, das Blut in den Hirngefäßen von Mäusen strömen zu sehen. Dabei wurde die Hirnfunktion der Mäuse keineswegs beeinflusst. Dies könnte eine deutliche Verbesserung in der Alzheimer- und Schlaganfallbehandlung zur Folge haben. Das Verfahren konnte bereits in den USA mit Erfolg an Schlaganfallpatienten getestet werden. Hierbei kann das gebündelte Licht durch die Schädeldecke in das Gehirn gelangen und die Energieproduktion der Nervenzellen anregen (Hong et al. 2012). Infrarotlaser finden neben der Neurologie auch in anderen Teilbereichen der Medizin, zum Beispiel in der Dermatologie oder Zahnmedizin ihren Einsatz. Studien über Effekte eines Infrarotlasers auf kognitive Funktionen gibt es bisher nicht. Dies haben wir uns in unserer Placebostudie zum Vorteil gemacht, um in den Probanden eine unvoreingenommene, hohe Erwartungshaltung durch verbale Manipulation evozieren zu können. 


\subsection{Hypothese}

Die aktuelle Studie soll dem Nachweis der Beeinflussung des instrumentellen Lernens durch Placebo- und Noceboeffekte dienen. Es soll untersucht werden, ob diese Effekte instrumentelles Lernen im Menschen fördern oder beeinträchtigen können. Die Placebo- und Noceboeffekte sollen in unserer Studie durch Sham- tDCS und fake- Infrarotlaserstimulation ausgelöst werden. Es ist bereits bekannt, dass transkranielle Gleichstromstimulation Einfluss auf die kognitiven Funktionen, inklusive dem instrumentellen Lernen, nehmen kann. Es ist zu vermuten, dass bereits eine induzierte Erwartungshaltung in den Probanden, evoziert durch verbale Manipulation in Form von Probandenvorinformationen zur Wirkung von tDCS und Infrarotlaserstimulation, die kognitiven Fähigkeiten beeinflussen kann, ohne dass eine reelle tDCS und Infrarotlaserstimulation angewendet wird. Den Probanden wird zuvor erläutert, dass die Stimulation die kognitiven Fähigkeiten entsprechend der Placebo- oder Nocebogruppe entweder verbessern oder verschlechtern kann. Entsprechend dieser verbalen Manipulation sollen Placebo-/Noceboeffekte ausgelöst werden. Dies kann nur gelingen, wenn die Vorinformationen zum Effekt der tDCS sehr überzeugend dargestellt werden.

Aus bereits durchgeführten Studien kristallisiert sich heraus, dass die Persönlichkeitsmerkmale der Probanden eine Rolle bei der Stärke der jeweiligen Placebo- und Noceboeffekte spielen sollen. Hierzu existieren jedoch erst wenige verifizierbare Ergebnisse. Der mögliche Zusammenhang zwischen Persönlichkeitsmerkmalen und der Stärke von Placebo- und Noceboeffekten soll auch in unserer Studie analysiert werden. 


\section{Material und Methoden}

\subsection{Ethikvotum}

Die vorliegende Studie wurde an der Universitätsmedizin Göttingen, Klinik für Klinische Neurophysiologie durchgeführt. Diese Studie ist offiziell registriert und hat das Votum des Ethikkomitees unter der Studiennummer 12/4/12 Amendement 2 erhalten.

\subsection{Probanden und Probandenauswahl}

Es wurden 32 Probanden durch Inserate in internen Studentenbörsen akquiriert (Durchschnittsalter: 24,66 \pm 2,54 Jahre; durchschnittliche Schul- und Studienzeit: 16,8 \pm 2,57 Jahre). Der mittlere Wert für die Händigkeit der Probanden lag bei 63,41 \pm 49,77 (SD).

Um zu vermeiden, dass der Menstruationszyklus der Frau einen Wechsel in neuronalen Belohnungsprozessen (Dreher et al. 2007) auslöst, wurden für diese Studie lediglich männliche Versuchspersonen rekrutiert. Während der jeweiligen Studiendauer nahm keiner der Probanden gleichzeitig an einem anderen Experiment teil. Insgesamt erklärten acht Probanden, dass sie in früheren Experimenten mit nicht- invasiven Hirnstimulationstechniken teilgenommen haben. Keiner der Teilnehmer durfte über Chinesisch- beziehungsweise Japanischkenntnisse verfügen. Diese Regelung diente dem Ausschluss etwaiger Kenntnisse, die eventuell die Aufgabenstellung vereinfachen könnten. Vor Start des Experimentes durchliefen die Probanden ein neurologisches Screening, welches von einem Neurologen der Klinik für Klinische Neurophysiologie der Universitätsmedizin Göttingen durchgeführt wurde. Dieser hatte keine Einblicke in das Ziel der Studie.

Die folgenden Ausschlusskriterien wurden benutzt:

- Alter $<18$ oder $>30$ Jahre

- Hinweise auf eine chronische Erkrankung oder Residuen einer neurologischen Erkrankung in der Vorgeschichte

- Herzschrittmacher, Tiefe Hirnstimulation

- Metallimplantate im Kopfbereich (in den Kopfbereich eingesetztes Metall, z.B. Clips nach Operation eines intrazerebralen Aneurysmas oder die Implantation einer künstlichen Hörschnecke) 
- Intrazerebrale Ischämien (Mangeldurchblutung des Gehirns/ Schlaganfall) oder Hirnblutungen in der Vorgeschichte.

- Hinweise auf epileptische Anfälle in der Vorgeschichte.

- Zustand nach Schädelhirntrauma mit Bewusstseinsverlust

- Vorliegen einer gravierenden internistischen oder psychiatrischen Vorerkrankung

- Alkohol-, Medikamenten- und Drogenabhängigkeit

- Rezeptive oder globale Aphasie

- Gleichzeitige Teilnahme an einer anderen wissenschaftlichen Studie

Auch Raucher wurden von dem Experiment ausgeschlossen, da die Konzentration während der Aufgaben nachlassen könnte. Außerdem wurden die Probanden gebeten, mindestens 3 Stunden vor Experimentbeginn keinen Kaffee zu trinken.

\subsection{Studiendesign}

In dieser Studie wurde den Teilnehmern und der Datensammlerin mitgeteilt, dass zwei Arten von Stimulation verwendet werden würden. Eine Stimulation würde die kognitive Leistungsfähigkeit der Teilnehmer verbessern, während die andere Stimulation sie beeinträchtigen würde. In Wirklichkeit erhielten alle Teilnehmer nur eine Sham- tDCS und eine fake-Infrarot-Laserstimulation. Diese Information war jedoch den Versuchsteilnehmern und der Datensammlerin unbekannt.

Subjektive Beeinflussungen von Seiten der Probanden und der Versuchsleiterin konnten infolgedessen ausgeschlossen werden. Somit konnte ein bedeutend höheres wissenschaftliches Niveau erreicht werden.

Des Weiteren wusste auch der Neurologe, der die Voruntersuchung durchführte, nichts von dem eigentlichen Anliegen der Studie. Alle Beteiligten gingen davon aus, dass sie an einer Studie zum Thema „Einfluss transkranieller Stromstimulation und Laserstimulation auf die kognitiven Funktionen" teilnahmen. 


\subsection{Paradigma}

Es wurde eine probabilistische Lern- und Entscheidungsfindungsaufgabe verwendet. Hierbei handelt es sich um eine bereits gut charakterisierte Aufgabe an Menschen (Frank et al. 2007; Jocham et al. 2011; Klein et al. 2007). Die Aufgabe besteht aus einer Lernphase und einer anschließenden Entscheidungsphase (Testphase). Während der Lernphase werden den Teilnehmern immer wieder drei Symbolpaare vorgestellt (AB, CD und EF). Hierbei handelt es sich um chinesische Schriftzeichen (Klein et al. 2007). Die Aufgabe der Teilnehmer ist es herauszufinden, welches Zeichen das Beste in jedem Paar ist. Dies geschieht über Versuch und Irrtum. Nach jeder Entscheidung erhalten die Teilnehmer eine Rückmeldung. Die Probanden wissen jedoch nicht, dass jedem Symbol ein fester Prozentsatz zugeordnet ist (A/B-80/20\%; C/D-70/30\% und E/F-60/40\%). Die Lernphase besteht aus vier Versuchsblöcken; jeder Block enthält 60 Wiederholungen mit AB, CD und EF-Paaren in einer zufälligen Reihenfolge. Die Präsentation jedes Symbolpaares kann auch seitenverkehrt auftreten; das heißt Symbolpaar AB oder BA kann vorliegen.

Jeder Versuch beginnt mit einem Fixationskreuz (0,3 s), im Anschluss erfolgt die Abbildung des Symbolpaares. Die Teilnehmer hatten maximal 1,7s Zeit zu reagieren, im Anschluss wurde das ausgewählte Symbol für 0,5s hervorgehoben. Nun schloss sich die Rückmeldung (0,5 s) an. Jeder Versuch dauerte somit 3,5 s (Abbildung 1).

Um die Motivationsebene zwischen den Behandlungsbedingungen konstant zu halten, wurden die Teilnehmer darüber informiert, dass sie 5 Euro für jede angefangene Stunde erhalten, plus 0,01 EUR für jede richtige Entscheidung (durch grünes Smiley- Gesicht und 0,01 EUR Schild gekennzeichnet). Bei inkorrekter Antwort bekam der Proband 0 EUR (rotes trauriges Gesicht und 0 EUR-Zeichen). Wenn man zu spät geantwortet hat, erschien ein gelbes Gesicht und die Bemerkung: „zu spät”. Nach einer kurzen Pause begann die Testphase. Die Testphase bestand aus Entscheidungen über alle möglichen Kombinationen der Symbole aus der Lernphase; dies bedeutet, dass diese Phase fünfzehn neue Kombinationen von Symbolen beinhaltet, die nicht während der Lernphase unter den drei alten Kombinationen (AB, CD und EF) anwesend waren (z.B. AF oder CE). Die Symbolpaare wurden zwölfmal wiederholt, was zu insgesamt 180 Entscheidungen führte. Jedes Symbolpaar war für die links- und rechtsseitige Darstellung ausgeglichen. In dieser Phase bekamen die Teilnehmer kein Feedback mehr. Ihnen wurde jedoch im Vorfeld erklärt, dass sie auch bei dieser Aufgabe eine leistungsabhängige Vergütung von 1 Cent pro korrekter Antwort bekommen würden. Dies galt sowohl für die Teilnehmer der Placebo- als auch der Nocebogruppe. 


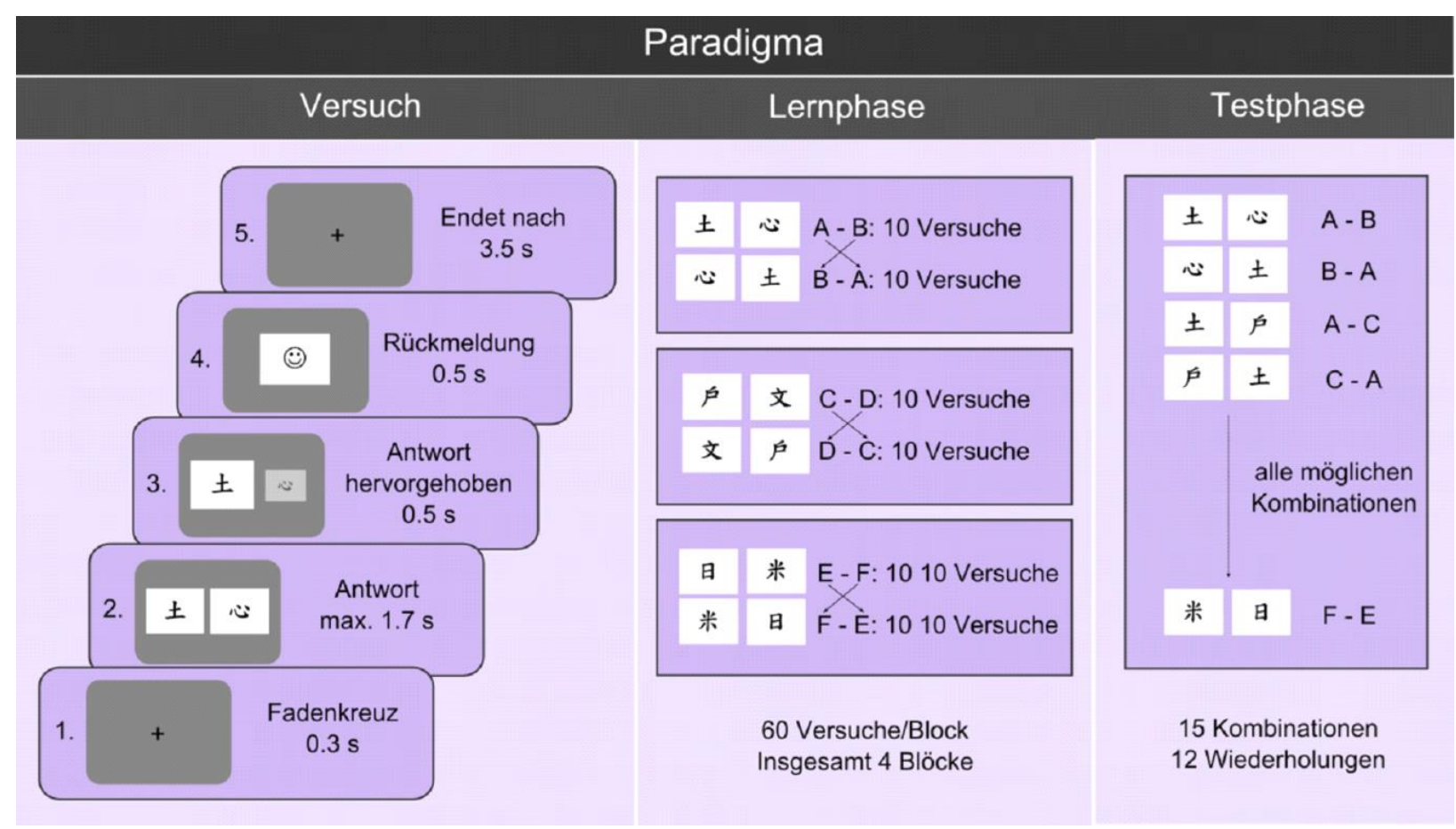

Abbildung 1. Paradigma: Versuchsaufbau, Lernphase mit 60 Versuchen je Block, insgesamt 4 Blöcke, Testphase mit allen möglichen Kombinationen der Schriftzeichen und 12 Wiederholungen

\subsection{Gruppen}

Bei dem durchgeführten Experiment gab es zwei separate Gruppen: eine Placebo- (Durchschnittsalter: 23,81 \pm 2,51 Jahre) und eine Nocebogruppe (Durchschnittsalter: 25,5 \pm 2,34 Jahre). Jede Gruppe umfasste 16 Probanden.

Die Zuordnung der 32 Teilnehmer der Studie erfolgte randomisiert. Mit Hilfe des Programmes „Phyton random. Suffle command“ wurden zwei zufällige Gruppen mit je 16 Teilnehmern gebildet. Gruppe X entsprach der Placebogruppe, Gruppe Y entsprach der Nocebogruppe. Daher verwendet die Studie das Design einer randomisierten Doppelblindstudie. Die Teilnehmer 3,5,6,10,12,13,17,18,19,21,22,24,25,27,29 und 31 gehörten der Placebogruppe an, die Teilnehmer 1,2,4,7,8,9,11,14,15,16,20,23,26,28,30 und 32 wurden somit der Nocebogruppe zugeordnet. Die Nummerierung der Teilnehmer erfolgte chronologisch nach ihrem Erscheinungsdatum.

Hinsichtlich Gesundheitszustand und Alter gab es keine signifikanten Unterschiede in beiden Gruppen. Die Probanden der Placebogruppe und Nocebogruppe hatten an beiden Tagen einen nahezu identischen Sitzungsablauf. Eine Differenz zwischen der Placebo- und Nocebogruppe stellte die prozentuale Verteilung der wahrscheinlich korrekten Antwort aus den Symbolpaaren während 
der zweiten Lernphase dar. Hierauf wird später näher eingegangen.

Ein weiterer Unterschied zwischen den Teilnehmern der Placebogruppe und Nocebogruppe lag in der Stimulationsinformation, die sie direkt vor der Stimulation erhielten (siehe Anlage 1). Sinngemäß erfuhren in dieser Information die Teilnehmer der Placebogruppe, dass durch die nun folgende Stimulation die kognitive Leistung um bis zu 25 Prozent gesteigert werden kann. Dieser Effekt hält etwa 30 Minuten an. Im Anschluss sollte der Proband schätzen, welche Leistungsveränderung in Prozent bei ihm eintreten könnte. Im späteren Verlauf des ersten Untersuchungstages wurde der Proband gefragt, um welchen Prozentsatz seine Leistung schätzungsweise gestiegen ist.

Analog dazu bekamen die Teilnehmer der Nocebogruppe unmittelbar vor der Stimulation ein Informationsblatt vorgelegt, welches beinhaltete, dass die Stimulation die kognitive Leistung um bis zu 25 Prozent verringert. Auch hier sollten die Probanden schätzen, welche Auswirkung in Prozent die Stimulation bei ihnen haben könnte. Auch die Probanden der Nocebogruppe wurden später gefragt, um welchen Prozentsatz ihre Leistung schätzungsweise gesunken ist.

\subsection{Stimulation}

Nach der Übungsphase erhielten die Probanden beider Gruppen die jeweilige Stimulationsinstruktion. Währenddessen bereitete die Versuchsleiterin die Elektroden vor. Hierzu wurde ein dünner Film einer Elektrodenpaste (Ten20 Conductive Paste; WEAVER and Company, USA) auf die beiden Elektroden aufgetragen. Zuvor wurden den Probanden EEG-Hauben aufgesetzt, um die genaue Platzierung der Elektroden zu markieren. Nach erfolgter Markierung wurde die Haube wieder abgenommen und die Elektroden an entsprechender Stelle positioniert. Die anodale Elektrode wurde mit der Elektrodenpaste auf F3 fixiert, die kathodale Elektrode auf F4 (internationales 10/20 EEG-System). 


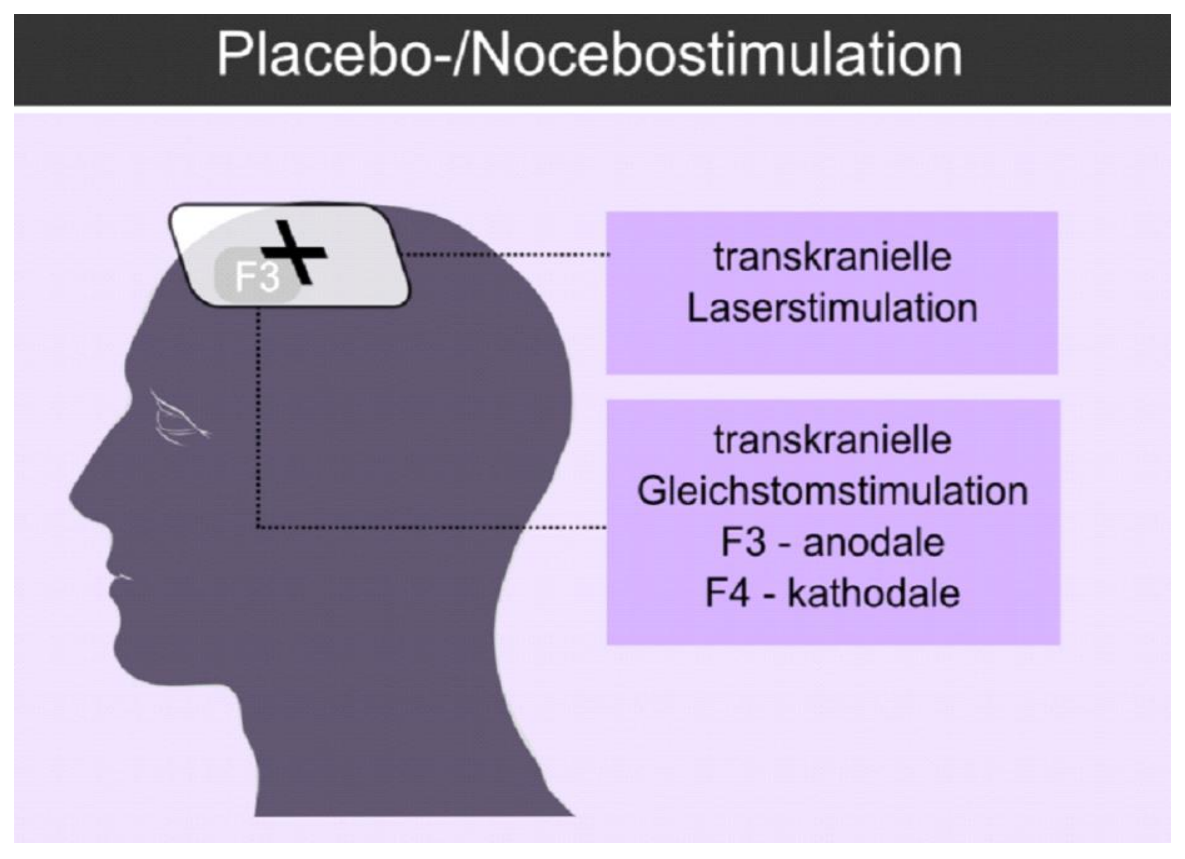

Abbildung 2. Placebo-/Nocebostimulation : Elektrodenposition; anodale Elektrode auf F3, kathodale Elektrode auf F4 (internationales 10/20 EEG-System)

Für die Stimulation wurde der DC-Stimulator ${ }^{\circledR}$ der neuroConn GmbH Ilmenau verwendet (vgl. Abbildung 3). Dieser CE- zertifizierte Stimulator dient der neurowissenschaftlichen Forschung. Neben der transkraniellen DC- und AC- Stimulation ermöglicht er zusätzlich eine Sham- Stimulation. Diese eignet sich besonders gut für Doppelblindstudien. 


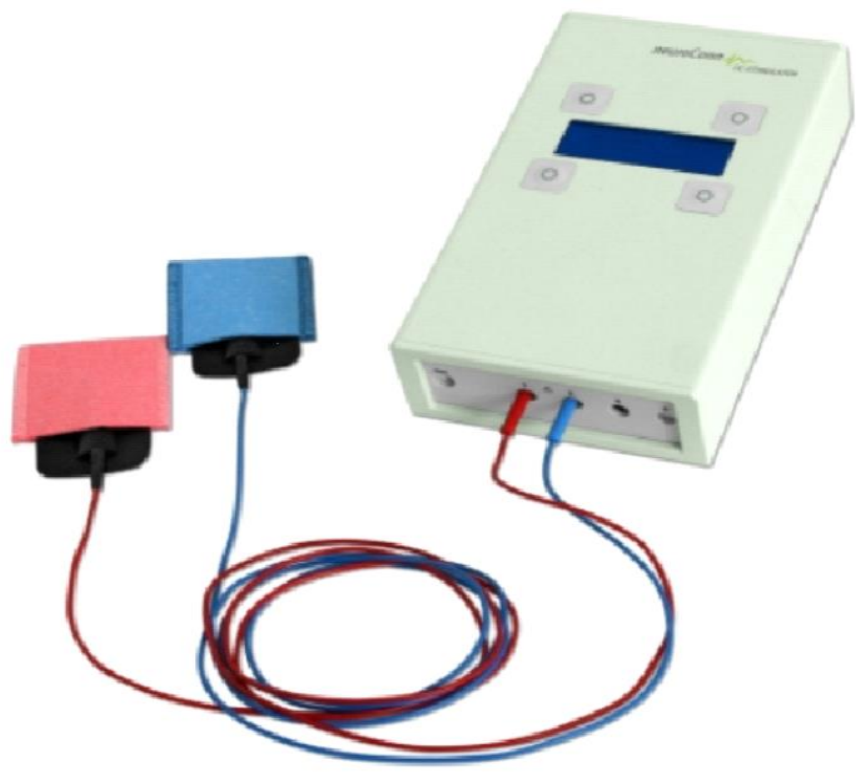

Abbildung 3. Gleichstromstimulator mit anodaler (rot) und kathodaler (blau) Elektrode, NeuroConn GmbH. (Quelle: http://www.neuroconn.de/dc-stimulator/)

Sowohl die Teilnehmer der Placebo- als auch der Nocebogruppe wurden mit entsprechender Sham- tDCS stimuliert. Die Stimulation dauerte eine Minute an. Diese Zeit gliederte sich in $20 \mathrm{~s}$ ,ramp up', 30 s 1 mA Stimulation und 10 s ,ramp down‘. Diese Stimulationsmethode wurde gewählt, damit die Probanden ein leichtes Kribbeln unter den Elektroden verspürten und somit keinen Verdacht schöpfen konnten, dass es sich um keine tatsächliche Gleichstromstimulation handelte.

Auch die Laserstimulation war eine reine fake-Stimulation. Diese wurde mit einem Infrarotlaser durchgeführt, der neben der Neurologie auch in anderen Teilbereichen der Medizin, zum Beispiel in der Dermatologie oder Zahnmedizin Einsatz findet. Die verbale Manipulation zur Verwendung des Infrarotlasers wurde eingesetzt, da bereits zahlreiche Studien zum Thema „Einfluss von tDCS auf kognitive Funktionen“ existieren. Die Probanden sowie die Versuchsleiterin hätten somit im Vorfeld Informationen aus dem Internet über die Methodik sammeln können. Durch die Einbeziehung eines Infrarotlasers, über den bis zum aktuellen Zeitpunkt keine Studien im Zusammenhang mit kognitive Funktionen existieren, konnte das Ziel der Studie als bedeutend interessanter dargestellt werden. Da es sich um ein unbekanntes Verfahren handelt, sollte die Erwartungshaltung, ausgelöst durch die verbale Manipulation, noch verstärkt werden.

Des Weiteren bekam auch die Versuchsleiterin Instruktionen über die Stimulation. Für die fake- Infrarotlaserstimulation war ein zweiter Experimentator notwendig. Diese Maßnahmen dienten 
dazu, dass auch die Versuchsleiterin davon ausging, dass es sich um eine reale transkranielle Gleichstrom- sowie Infrarotlaserstimulation handelte. Die Probanden der Placebogruppe gingen davon aus, dass die Stimulation ihre kognitive Leistung verbessern würde, die Nocebogruppe glaubte, dass die Stimulation die Leistung verschlechtert.

\subsection{Versuchsdurchführungen}

Die Studie fand pro Proband an zwei aufeinander folgenden Tagen statt. Die erste Sitzung umfasste einen Zeitrahmen von circa zwei Stunden, die zweite Sitzung dauerte eine Stunde.

\subsubsection{Beschreibung der eingesetzten Fragebögen}

Die Originalfragebögen können im Anhang nachgeschlagen werden. $B I S / B A S$

Im Rahmen der hier vorgestellten Arbeit füllten die Probanden vor Experimentbeginn einen BIS/BAS- Fragebogen mit 24 Items aus. Der Fragebogen enthält eine Reihe von Feststellungen, mit denen man sich selbst beschreiben kann. Diese Feststellungen können genau zutreffen, eher zutreffen, eher nicht oder gar nicht auf die Probanden zutreffen. Die entsprechenden Entscheidungen der Probanden wurden angekreuzt. Somit sollen bestimmte Verhaltensmuster und Persönlichkeitseigenschaften der Probanden detektiert und im weiteren Verlauf ein eventueller Zusammenhang mit der Stärke von Placebo-/Noceboeffekten ermittelt werden. Folgende Items wurden verwendet:

1. Eine eigene Familie ist die wichtigste Sache im Leben.

2. Sogar wenn mir etwas schlimmes bevorsteht, bin ich selten nervös oder ängstlich.

3. Ich strenge mich besonders an, damit ich erreiche was ich möchte.

4. Wenn mir etwas gut gelingt, bleibe ich sehr gern bei der Sache.

5. Ich bin immer bereit, etwas neues zu versuchen, wenn ich denke, dass es Spaß machen wird.

6. Es ist wichtig für mich, wie ich gekleidet bin.

7. Wenn ich erreiche, was ich will, bin ich voller Energie und Spannung.

8. Kritik und Beschimpfungen verletzen mich ziemlich stark.

9. Wenn ich etwas haben will, tue ich gewöhnlich alles, um es zu bekommen.

10. Ich werde oft Dinge nur deshalb tun, weil sie Spaß machen könnten.

11. Es ist schwierig für mich, Zeit für solche Dinge wie Friseurbesuche zu finden.

12. Wenn ich eine Chance sehe, etwas Erwünschtes zu bekommen, versuche ich sofort mein 
Glück.

13. Ich bin ziemlich besorgt oder verstimmt, wenn ich glaube oder weiß, dass jemand wütend auf mich ist.

14. Wenn ich eine Gelegenheit für etwas sehe, das ich mag, bin ich sofort voller Spannung.

15. Ich handle oft so, wie es mir gerade in den Sinn kommt.

16. Wenn ich glaube, dass mir etwas Unangenehmes bevorsteht, bin ich gewöhnlich ziemlich unruhig.

17. Ich wundere mich oft über das menschliche Verhalten.

18. Wenn mir etwas Schönes passiert, berührt mich das sehr stark.

19. Ich bin besorgt, wenn ich glaube, dass ich eine wichtige Sache schlecht gemacht habe.

20. Ich brauche Abwechslung und neue Erfahrungen.

21. Wenn ich etwas erreichen will, verfolge ich hartnäckig mein Ziel.

22. Verglichen mit meinen Freunden habe ich sehr wenig Ängste.

23. Ich fände es sehr aufregend, einen Wettbewerb zu gewinnen.

24. Ich habe Angst, Fehler zu machen.

Die Items 2,8,13,16,19,22,24 dienen zur Aufstellung der BIS- Skala, die Items 3,9,12 und 21 bestimmen BAS Drive, die BAS Fun Seeking Skala bedient sich der Items 5,10,15 und 20 und die Items 4,7,14,18 und 23 dienen der Erstellung der BAS Reward Responsiveness Skala. Die Items 1,6, 11 und 17 sind so genannte Füllitems. Füllitems dienen dem alleinigen Zweck, dass der Proband das Testziel nicht deutlich erkennen kann.

\section{Edinburgh Handedness Skala}

Zur Bestimmung der Händigkeit der Probanden wurde die Edinburgh Handedness Skala (Oldfield 1971) verwendet. Hierbei handelt es sich um eine Tabelle mit zehn Aktivitäten (schreiben, zeichnen, werfen, schneiden mit einer Schere, Zahnbürste benutzen, schneiden mit einem Messer, einen Löffel benutzen, mit einem Besen kehren, ein Streichholz anzünden und eine Schachtel öffnen). Der Proband hatte vor Beginn des Experimentes die Aufgabe, jeweils anzukreuzen, ob er die Aktivität mit der rechten oder linken Hand ausübt. Wenn er eine Hand stark bevorzugt, war ++ auf der entsprechenden Seite einzutragen. Sollte der Proband keine der beiden Hände für die Aktivität bevorzugen, wird ein + jeweils in die rechte und linke Spalte eingetragen. 


\section{Fragebogen über Schulbildung}

Zur Ermittlung eines Zusammenhangs zwischen dem Lösen der Aufgaben und dem Bildungsgrad der Teilnehmer wurden in der Vorbereitungsphase folgende Fragen gestellt:

- Wie viele Jahre haben Sie mit Schule, Ausbildung und Studium verbracht? (in Jahren):

- Was studieren Sie bzw. welche Ausbildung haben Sie absolviert?

\section{Vorerfabrungen mit anderen Experimenten}

In der aktuellen Studie galt eine Vorerfahrung mit anderen tDCS- Studien als Ausschlusskriterium, um zu verhindern, dass ein Proband eine Placebostudie vermuten könnte. Folgende Fragen wurden in der Vorbereitungsphase gestellt:

- Haben Sie bereits in einem anderen Experiment mit elektrischer Stimulation teilgenommen?

- Nehmen Sie zurzeit an einem anderen Experiment Teil?

- Haben Sie bereits in einem anderen Experiment in unserer Abteilung teilgenommen?

\section{Kopfschmerzen}

Die Frage nach Kopfschmerzen wurde sowohl am ersten als auch am zweiten Tag in der Vorbereitungsphase gestellt. Dies diente der Detektion eines eventuellen Zusammenhangs zwischen Kopfschmerzen als Nebenwirkung auf die Stimulation am Vortag. Wenn die Probanden mit „JA“ antworten, folgt eine Einschätzung der Kopfschmerzen nach der Häufigkeit (von täglich bis weniger als einmal im Monat) und Intensität (von gering bis nicht aushaltbar).

\section{Schlafverhalten}

Folgende Fragen zum Schlafverhalten wurden ebenfalls gleichermaßen am ersten und zweiten Versuchstag gestellt. Ein eventueller Zusammenhang zwischen dem Experiment und eventuellen Veränderungen im Schlafverhalten kann somit abgeleitet werden. 
- Wie viele Stunden haben Sie geschlafen in der letzten Nacht?

- Bitte schätzen Sie, wie gut Sie geschlafen haben auf einer Skala von 1 bis 5 (1: sehr schlecht -5 : sehr gut)

- Wie viele Minuten brauchten Sie zum Einschlafen?

- Wie oft sind Sie aufgewacht während der letzten Nacht?

\section{LOT-R}

In der aktuell vorgestellten Studie wurde der LOT- R Fragebogen mit zehn Items von jedem Probanden vor dem Experiment ausgefüllt. Die Probanden können jeweils zwischen fünf Antwortmöglichkeiten auswählen. Diese Feststellungen können von voll und ganz bis überhaupt nicht zutreffen. Die entsprechenden Entscheidungen der Probanden werden angekreuzt. Auch mit diesem Fragebogen sollen Charaktereigenschaften der Probanden detektiert werden. Diese Charaktereigenschaften könnten Einfluss auf die Wirkung von Placebo/- und Noceboeffekten besitzen. Der LOT-R Test soll der Aufschlüsselung des Optimismusverhaltens der Probanden dienen.

1. Auch in ungewissen Zeiten erwarte ich normalerweise das Beste.

2. Es fällt mir leicht, mich zu entspannen.

3. Wenn bei mir etwas schief laufen kann, dann tut es das auch.

4. Meine Zukunft sehe ich immer optimistisch.

5. In meinem Freundeskreis fühle ich mich wohl.

6. Es ist wichtig für mich, ständig beschäftig zu sein.

7. Fast nie entwickeln sich die Dinge nach meinen Vorstellungen.

8. Ich bin nicht allzu leicht aus der Fassung zu bringen.

9. Ich zähle selten darauf, dass mir etwas Gutes widerfährt.

10. Alles in allem erwarte ich, dass mir mehr gute als schlechte Dinge widerfahren.

Die Items 2, 5, 6 und 8 dienen der Aufschlüsselung des Optimismusverhaltens der Probanden. Die Items 3, 7 und 9 sollen Aufschluss über das Pessimismusverhalten der Probanden geben. Die Items 1, 4 und 10 gelten als Füllitems. 


\subsubsection{Erste Sitzung - Vorbereitungen}

Jeder Proband hatte die gleichen Grundvoraussetzungen. Am ersten Versuchstag wurden den Probanden beider Gruppen allgemeine Informationen über die Studie vorgelegt (siehe Anlage 2). Hierbei wurde darauf hingewiesen, zu welchem Zweck das Experiment durchgeführt wird, mögliche Risiken und Nebenwirkungen erklärt sowie der Stimulator beziehungsweise die Stimulationsmethode kurz erläutert. Alle Beteiligten gingen laut Vorinformationen davon aus, tatsächlich mit transkranieller Gleichstrom- und Infrarotlaserstimulation für eine Minute stimuliert zu werden. Außerdem wurden die Probanden darüber aufgeklärt, dass sie das Experiment jederzeit beenden könnten.

Vor Beginn des Experimentes am ersten Tag füllten alle Teilnehmer nach der Einverständniserklärung einen BIS/BAS-Fragebogen (Anlage 3), die Edinburgh Handedness Skala von Oldfield 1971 (Anlage 4), einen Fragebogen über Schulbildung, Vorerfahrungen mit anderen Experimenten, Schlafverhalten und Kopfschmerzen (Anlage 5) sowie einen LOT-R Fragebogen (Anlage 6) aus. Im nächsten Schritt fand die neurologische Untersuchung statt. Während dieser Untersuchung wurde die Anamnese des Teilnehmers aufgenommen sowie Okulomotorik und Gangverhalten überprüft. Zudem wurde eine Blutdruckmessung durchgeführt.

Nach der neurologischen Untersuchung begann der erste Versuchstag. Dieser umfasste einen Gesamtzeitrahmen von circa zwei Stunden. Zuerst wurde den Probanden eine allgemeine Experimentanleitung vorgelegt (Anlage 7). In dieser fand sich eine Zusammenfassung über den zeitlichen Ablauf der zwei Versuchstage sowie die Aufgabenstellung. Um sicherzustellen, dass der Proband die Aufgabenstellung verstanden hat, wurde ein kurzer Pretest durchgeführt. Hierbei waren drei Fragen zu beantworten (siehe Anlage 8). Nach bestandenem Test fand eine erste kurze Übung am PC statt. Diese wurde einmal von der Versuchsleiterin vorgeführt und sollte im nächsten Durchlauf durch den Probanden ausgeführt werden. Auf dem Bildschirm erschienen bereits Symbolpaare. Hierbei handelte es sich lediglich um geometrische Formen, nicht um die chinesischen Schriftzeichen der späteren Aufgabe. Diese Übung diente dazu, dass sich der Proband mit der ihm vorliegenden Tastatur vertraut machte. Außerdem sollte der Proband nach der Übung abschätzen können, wie viel Zeit ihm zur jeweiligen Entscheidungsfindung zur Verfügung stand. Dies waren 1.7 Sekunden. Auf der Tastatur befanden sich neben einigen unbeschrifteten Tasten eine L-Taste und eine R-Taste. Es wurde darauf geachtet, dass der Versuchsteilnehmer die L-Taste nur mit dem linken und die R-Taste nur mit dem rechten Zeigefinger betätigt. 


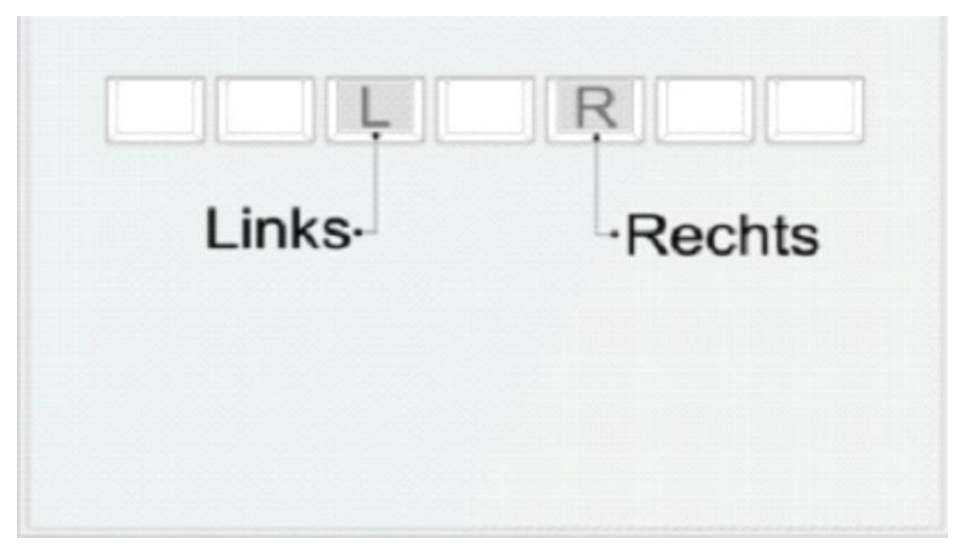

Abbildung 4. Tastatur zur Aufgabenlösung mit L-Taste und R-Taste; die L- Taste durfte nur mit dem linken Zeigefinger betätigt werden, die R- Taste entsprechend nur mit dem rechten Zeigefinger.

Den Probanden wurde erklärt, dass man sich jeweils für das rechte oder linke Symbol, welches im Paar auf dem Bildschirm erschien, entscheiden sollte. Diese Entscheidung sollte rein intuitiv erfolgen. Bei korrekter Entscheidung erhielt der Proband eine positive Rückmeldung (grün), bei falscher Entscheidung eine negative Rückmeldung (rot). Bei Nichteinhaltung der Zeitbeschränkung erfolgte der Hinweis dass zu spät geantwortet wurde (gelb). Die identischen Rückmeldungen wurden dann auch während der ersten und zweiten Lernphase verwendet. Im Anschluss konnte der Proband die tatsächlichen Schriftzeichen, die in der folgenden ersten Lernphase verwendet wurden, vorab betrachten. Alle sechs Schriftzeichen erschienen einzeln für fünf Sekunden auf dem Bildschirm. In einem zweiten Durchlauf wurden sie noch einmal wiederholt. Dies diente dazu, dass sich der Proband schon einmal mit den Schriftzeichen vertraut machen konnte.

\subsubsection{Erste Sitzung - Hauptexperiment}

Nun erfolgte die Lernphase eins. In der ersten Lernphase war die prozentuale Wahrscheinlichkeit für alle Symbolpaare der Placebo-und Nocebogruppe identisch (A/B- 80/20\%; C/D70/30\% und E/F- 60/40\%; Standardbelohnung). Gleiches gilt für die erste Testphase. Vorab bekamen die Probanden eine genaue Beschreibung dieser Übung. Im Anschluss an die Lernphase erfolgte nach kurzer Pause die Testphase. Auch die Testphase wurde zuvor genau erklärt. Genauere Erläuterungen hierzu finden sich im Abschnitt 2.4.

In der zweiten Lernphase, die sich an die Sham-Stimulation anschloss, führten die Probanden eine identische Aufgabenstellung wie in der ersten Lernphase am PC aus. 
Es wurden sechs neue Schriftzeichen verwendet. Außerdem unterschied sich der Prozentsatz der Wahrscheinlichkeit der korrekten Antworten in beiden Gruppen. In der zweiten Lernphase lag in der Placebogruppe eine manipulierte Belohnung vor: (A/B-90/10\%; C/D-90/10\% und E/F80/20\%). Durch die deutlich vereinfachte Aufgabenstellung sollten die Probanden davon ausgehen, dass die Stimulation ihre kognitive Leistung zum Positiven verändert hat. Somit sollte die Erwartungshaltung der Probanden in Bezug auf die Wirksamkeit der Stimulation verstärkt werden.

Auch der Prozentsatz für die Wahrscheinlichkeit der korrekten Antwort während der zweiten Lernphase der Nocebogruppe wurde manipuliert (A/B-60/40\%; C/D-60/40\% und E/F-55/45\%). Durch die deutlich erschwerte Aufgabenstellung im Vergleich zur ersten Lernphase sollen die Probanden davon ausgehen, dass sich durch die Stimulation die kognitive Leistung deutlich verschlechtert hat. Somit soll entsprechend die Erwartungshaltung der Probanden beeinflusst werden. Im Anschluss an die zweite Lernphase wurde ein Fragebogen zu den möglichen Nebenwirkungen von allen Probanden ausgefüllt.

\section{PLACEBOGRUPPE}

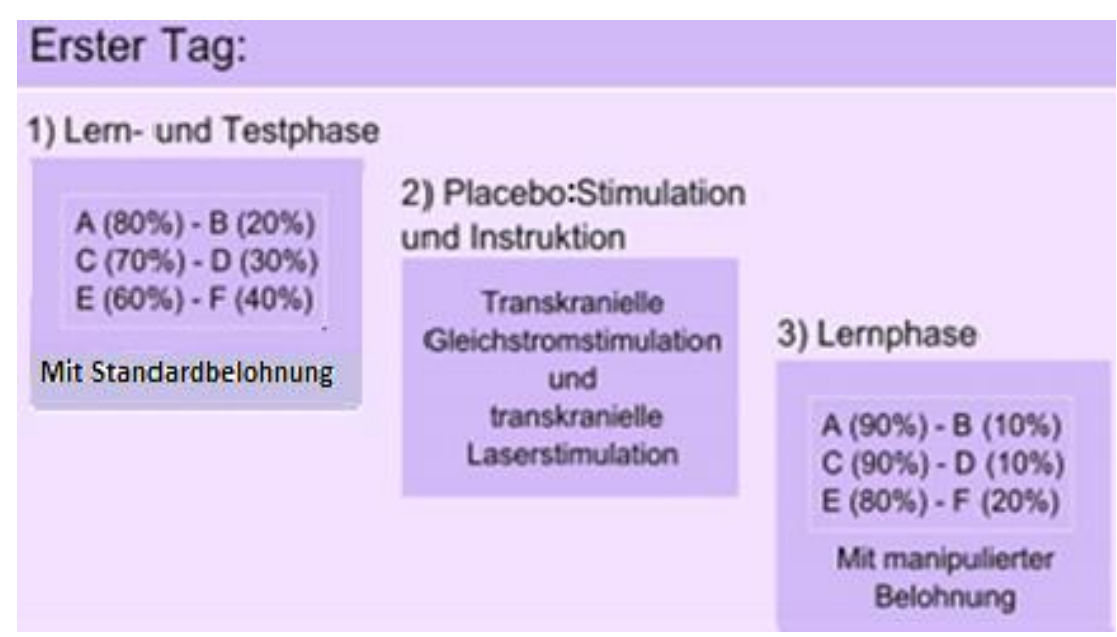

Abbildung 5. Placebogruppe: Sitzungsablauf erster Tag; 1) Erste Lern- und Testphase mit Standardbelohnung, 2) Placebo: Stimulation und Instruktion, 3) Zweite Lernphase mit manipulierter Belohnung. 


\section{NOCEBOGRUPPE}

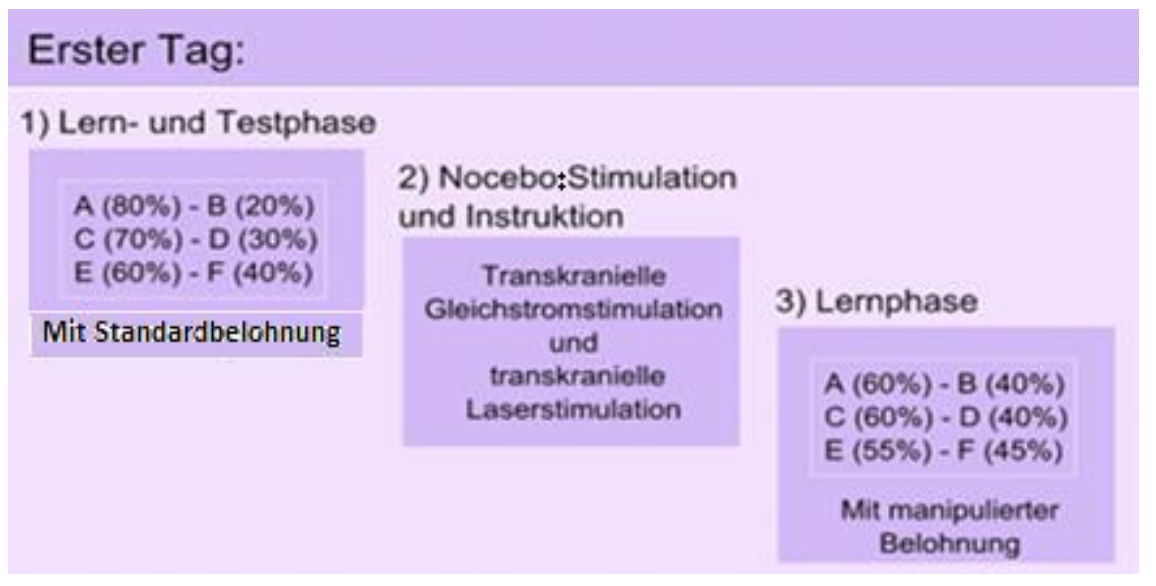

Abbildung 6. Nocebogruppe: Sitzungsablauf erster Tag; 1) Erste Lern- und Testphase mit Standardbelohnung, 2) Nocebo: Stimulation und Instruktion, 3) Zweite Lernphase mit manipulierter Belohnung.

\subsubsection{Zweite Sitzung}

Die zweite Sitzung am Folgetag umfasste einen Zeitrahmen von circa einer Stunde. Das Experiment startete analog zum ersten Versuchstag für die Teilnehmer der Placebo- und Nocebogruppe; (Fragebogen über Schlafverhalten, Kopfschmerzen, Kaffeekonsum etc. ausfüllen, die Übung am PC ausführen, die Information über die Lernphase lesen und die neuen Schriftzeichen betrachten).

Nach den vorbereitenden Maßnahmen erfolgten bereits die Stimulationsvorbereitung und die Stimulation analog den Bedingungen des Vortages, jedoch ohne vorherige Lern-und Testphase. Nach der Stimulation vollzogen die Probanden beider Gruppen die Lernphase mit anschließender Testphase (siehe Paradigma). Analog zur ersten Lern- und Testphase am ersten Versuchstag lag auch hier eine Standard Belohnung vor (A/B- 80/20\%; C/D-70/30\% und E/F- 60/40\%; Standard Belohnung).

Im Anschluss an die Testphase wurde von den Probanden ein letzter Fragebogen zu möglichen Nebenwirkungen oder Auffälligkeiten ausgefüllt. Des Weiteren wurden alle Probanden in einem kurzen Abschlussgespräch gebeten, ihre Lernstrategie zu erläutern. Hierbei wurden die Probanden aufgefordert, noch einmal die Bildpaare, die sie an beiden Tagen gesehen hatten, zuzuordnen 
und eine Rangfolge über die Schriftzeichen (von sehr wahrscheinlich richtig zu eher unwahrscheinlich richtig) zu erarbeiten.

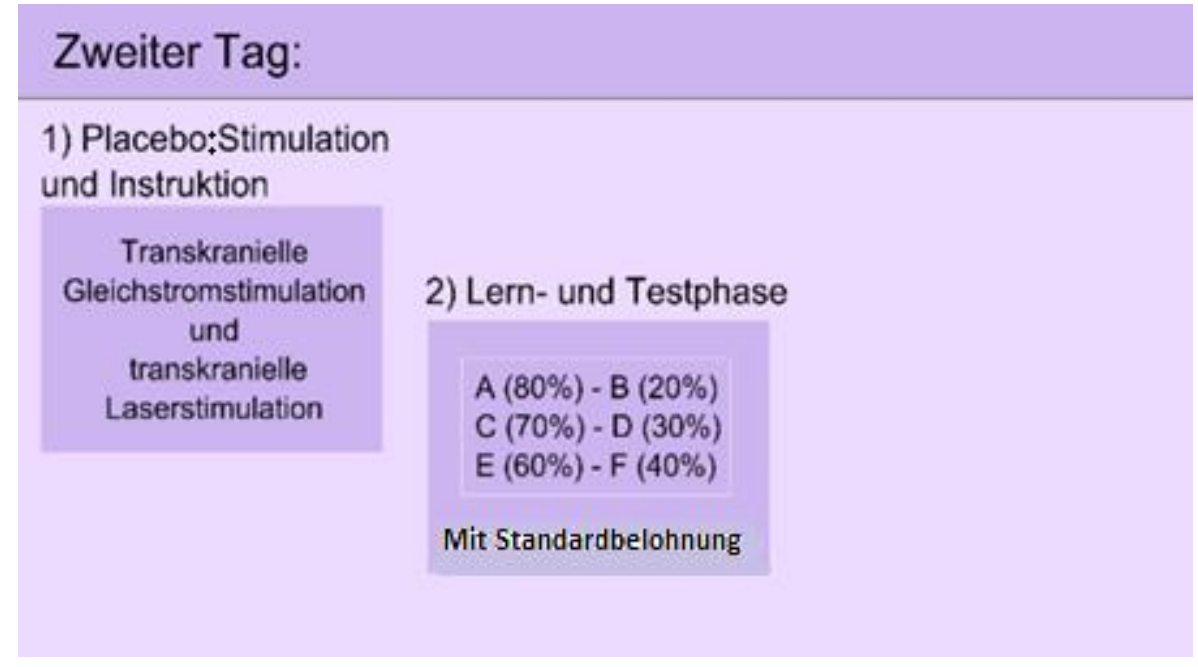

Abbildung 7. Placebogruppe: 2. Sitzung; 1) Placebo: Stimulation und Instruktion; 2) Lern- und Testphase mit Standardbelohnung.

\section{Zweiter Tag:}

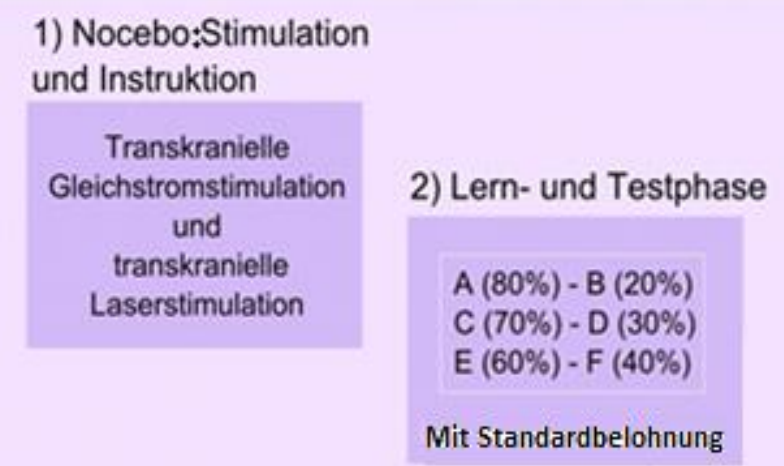

Abbildung 8. Nocebogruppe: 2. Sitzung; 1) Nocebo: Stimulation und Instruktion; 2) Lern- und Testphase mit Standardbelohnung 


\subsection{Aufwandsentschädigung}

Alle Probanden bekamen eine Aufwandsentschädigung. Im Anschluss an die zweite Sitzung wurden die Teilnehmer über die Höhe des finanziellen Ausgleichs für die verbrachte Zeit und ihre zusätzlichen Erträge während des Experiments informiert. Allerdings wussten weder die Versuchsleiterin, noch die Probanden, dass für jeden Versuchsteilnehmer eine identische Vergütung in Höhe von 8,50 EURO pro angefangene Stunde berechnet wurde. Diese Maßnahme verhinderte eine finanzielle Benachteiligung der Nocebogruppe.

\subsection{Datenerhebung und Statistik}

Alle Analysen wurden durch das statistische Programm R und R-Studio (Version 0.98.1091) durchgeführt. Nullhypothesen wurden durch den Gebrauch von $\mathrm{p}<-0,05$ ausgeschlossen. Für die aufzählungsabhängigen Variablen wurde der Mann-Whitney-Test benutzt. Die Bonferroni-HolmsKorrektur wurde für multiple Vergleiche verwendet. 


\section{Ergebnisse}

Die durchgeführte Studie mit dem Design einer randomisierten Doppelblindstudie diente dem Nachweis der Beeinflussung von Placebo-und Noceboeffekten auf die kognitive Lernleistung. Zum Aufbau einer Erwartungshaltung in den Probanden wurde eine verbale Manipulation über Sham- tDCS und fake- Infrarotlaserstimulation im Vorfeld gegeben. Das Experiment wurde von keinem der 32 Teilnehmer vorzeitig abgebrochen. Kein Teilnehmer vermutete die ShamStimulationen. Alle Sitzungen liefen planmäßig ohne Zwischenfälle ab.

Die Teilnehmer der Nocebogruppe wiesen im Vergleich zur Placebogruppe eine signifikant höhere Schulbildung auf $(\mathrm{t}=2,8617$; $\mathrm{df}=27,688$; $\mathrm{p}$ - value= 0,008). Die Werte wurden nach der Auswertung der Fragebögen zur Schulbildung ermittelt. Die Probanden der Nocebogruppe konnten durchschnittlich 17,9 Jahre nachweisen, die sie mit Schule und Ausbildung bzw. Studium verbracht haben, die Teilnehmer der Placebogruppe hingegen lediglich 15,63 Jahre im Durchschnitt. Es konnte jedoch kein Zusammenhang zwischen der Schulbildung und dem Lösen der Aufgaben festgestellt werden.

\subsection{Stimulationsinduzierte Hautempfindungen}

Im ersten Schritt wurden die subjektiv bewerteten Hautempfindungen der Teilnehmer zwischen den Placebo- und Nocebogruppen während der ersten und zweiten Sitzung gesondert verglichen. Im Allgemeinen waren beschriebene Hautbeschwerden in beiden Gruppen sehr gering. Nahezu alle Probanden empfanden ein leichtes Kribbeln unter den Elektroden, lediglich zwei Probanden gaben ein leichtes Brennen während der Stimulation an. Der Mann-Whitney-Wilcoxon-Test zeigte keinen signifikanten Unterschied in der ersten Sitzung zwischen den beiden Gruppen $(p=0,98)$. Allerdings fand sich ein marginal signifikanter Trend während der zweiten Sitzung $(\mathrm{W}=0,8 ; \mathrm{p}=$ 0,09). Hierbei erlebten die Teilnehmer der Nocebogruppe erhöhte Hautempfindungen. 


\begin{tabular}{cccc}
\hline \hline Sitzung & Placebo & Nocebo & $\boldsymbol{P}$-Wert \\
\hline \hline Erste & $0,93 \pm 1,33$ & $1,89 \pm 3,26$ & n.s. \\
Zweite & $0,47 \pm 1,55$ & $1,93 \pm 2,70$ & 0,09 \\
\hline \hline
\end{tabular}

Tabelle 1. Subjektive kutane Beschwerdeebene. Kein statistisch signifikanter Unterschied zwischen der Placebo- und Nocebogruppe in der ersten Sitzung; jedoch wurde ein marginal signifikanter Trend in Bezug auf die zweite Sitzung gefunden.

\subsection{Schlafqualität}

Aus den gesammelten Daten der Probandenfragebögen zum subjektiv bewerteten Schlafempfinden wurde ein Vergleich für die Placebo- und Nocebogruppe in der ersten und zweiten Sitzung erstellt. Die Datenermittlung erfolgte auf der Grundlage des Mann-Whitney-Wilcoxon-Tests. Es konnte kein Unterschied zwischen den beiden Gruppen in der Menge des Schlafes (beide ps $\geq 0,75$ ), in der Qualität des Schlafes (beide ps $\geq 0,79$ ), in der Zeit um einzuschlafen (beide ps = 1) und der Häufigkeit des Erwachens (beide ps $\geq 0,57$ ) in der ersten oder in der zweiten Sitzung festgestellt werden. Kein Proband beschrieb in der zweiten Sitzung einen Zusammenhang zwischen dem ersten Versuchstag und der Schlafqualität in der folgenden Nacht. Tabelle 2 zeigt eine Zusammenstellung dieser Ergebnisse. 


\begin{tabular}{|c|c|c|c|c|}
\hline & Sitzung & Placebo & Nocebo & P-Wert* \\
\hline \multirow[t]{2}{*}{$\begin{array}{c}\text { Schlafmenge in } \\
\text { Stunden }\end{array}$} & Erste & $7,37 \pm 0,88$ & $7,62 \pm 1,48$ & n.s. \\
\hline & Zweite & $7,61 \pm 1,00$ & $7,30 \pm 1,23$ & n.s. \\
\hline \multirow[t]{2}{*}{ Schlafqualität } & Erste & $3,50 \pm 0,73$ & $3,44 \pm 1,03$ & n.s. \\
\hline & Zweite & $3,50 \pm 1,21$ & $3,81 \pm 1,05$ & n.s. \\
\hline \multirow[t]{2}{*}{$\begin{array}{c}\text { Einschlafzeit in } \\
\text { Minuten }\end{array}$} & Erste & $22,22 \pm 17,57$ & $18,47 \pm 13,37$ & n.s. \\
\hline & Zweite & $16,56 \pm 11,21$ & $15,53 \pm 14,87$ & n.s. \\
\hline \multirow[t]{2}{*}{$\begin{array}{l}\text { Häufigkeit des } \\
\text { Erwachens }\end{array}$} & Erste & $1,06 \pm 1,73$ & $1,50 \pm 1,52$ & n.s. \\
\hline & $Z_{\text {weite }}$ & $1,03 \pm 1,13$ & $1,56 \pm 2,16$ & n.s. \\
\hline
\end{tabular}

Tabelle 2: Fragebogen zur Schlafqualität; Es wurde kein statistisch signifikanter Unterschied zwischen der Placebo- und Nocebogruppe der ersten oder der zweiten Sitzung in keiner der untersuchten Möglichkeiten gefunden. * Korrigiert für multiple Vergleiche.

\subsection{Erregungsniveau}

Des Weiteren wurde das subjektiv bewertete Erregungsniveau vor und nach der Stimulation zwischen der Placebo- und Nocebogruppe verglichen. Daten von der Liket Skala wurden in den Mann-Whitney-Wilcoxon-Test eingegeben. Hierbei ergaben sich keine signifikanten Unterschiede für alle Vergleiche (alle ps $\geq 0,4$ ). Somit bestätigt diese Analyse, dass sich das subjektiv berichtete Erregungsniveau der Teilnehmer zwischen den beiden Gruppen nicht unterscheidet. 


\begin{tabular}{lcccc}
\hline \hline Sitzung & Zeit & Placebo & Nocebo & P-Wert* \\
\hline Erste & Vorher & $7,94 \pm 1,29$ & $8,06 \pm 1,84$ & n.s. \\
& Nachher & $6,47 \pm 1,18$ & $6,81 \pm 1,94$ & n.s. \\
& & & & \\
\hline Zweite & Vorher & $6,75 \pm 1,84$ & $7,81 \pm 2,14$ & n.s. \\
& Nachher & $5,69 \pm 1,99$ & $6,62 \pm 1,82$ & n.s. \\
& & & & \\
\hline \hline
\end{tabular}

Tabelle 3. Subjektives Erregungsniveau. Es wurde kein statistisch signifikanter Unterschied zwischen den Placebo- und Nocebogruppen während der ersten oder der zweiten Sitzung in keiner der untersuchten Möglichkeiten gefunden.* Korrigiert für multiple Vergleiche.

\subsection{Der Einfluss von Placebo-und Nocebostimulation auf die kognitive Lernleistung}

Die Lernleistung der Probanden wurde während vier Blöcken eines Standardtests für Verstärkungslernen in jeder Sitzung beobachtet. Dabei verwendete man unabhängige Schriftzeichenpaare. Die Lernstrategie wurde analysiert indem die korrekten Daten von dem Übungsteil des Experiments mit einbezogen wurden. Es wurden folgende Einflussvariablen gewählt: drei Ebenen: (AB,CD, EF), vier Blocknummern (Ebenen: 1-4) und zwei Gruppen (Placebo und Nocebo).

Tag eins

Die Analyse des Wald-Tests bestätigte die erwartete Wirkung von Lernblöcken, das heißt, die Leistung wurde allmählich im Verlauf des Experiments gesteigert, wie durch den Block-Effekt angegeben. Ebenso wie erwartet, lag eine geringere Leistung für das Schriftzeichenpaar mit erhöhter Schwierigkeit vor. Die Analyse bestätigte weiterhin, dass es keinen Unterschied in der anfänglichen Lernleistung zwischen den beiden Gruppen vor dem Experiment gab. Somit kann bestätigt werden, dass das Bildungsniveau der Teilnehmer keinen Einfluss auf die Lösung der Aufgabenstellung hat. 
Odd Ratios $\quad 2,5 \% \quad 97,5 \%$

\begin{tabular}{cccc} 
CD Paat & 0,84 & 0,72 & 0,98 \\
EF Paar & 0,27 & 0,24 & 0,31 \\
Block 2 & 1,47 & 1,26 & 1,70 \\
Block 3 & 1,99 & 1,70 & 2,32 \\
Block 4 & 2,03 & 1,73 & 2,37 \\
Nocebogruppe & 0,93 & 0,83 & 1,04 \\
\hline \hline
\end{tabular}

Tabelle 4. Potenzierte Koeffizienten (Wahrscheinlichkeits-Verhältnisse) und Vertrauensbereiche am ersten Tag

\section{Zweiter Tag}

Ebenso wie am ersten Tag fand sich ein signifikanter Effekt der Block- und Stimuli-Paare. Die Analyse zeigte einen signifikanten Effekt für die Einflussvariable der Gruppe, das heißt, die Lernleistung verbesserte sich deutlich in der Placebogruppe im Vergleich zur Nocebogruppe. Die allgemeine Lernfähigkeit wurde für jeden einzelnen Probanden für Tag eins und Tag zwei getrennt berechnet. Durch Subtraktion der Ergebnisse von Tag eins und Tag zwei konnte die kognitive Lernleistung der Probanden beurteilt werden. Während positive Werte bedeuten, dass die kognitive Lernleistung der Teilnehmer sich vom ersten zum zweiten Tag verbesserte, zeigt ein negativer Wert das Gegenteil an. Hieraus kann abgeleitet werden, dass die Stimulationsinstruktion, bei der die Probanden zuvor erfuhren, dass die folgende Stimulation die kognitive Leistung verbessern werde, so in das Bewusstsein der Probanden eingedrungen ist, dass allein durch den Gedanken an die Leistungsverbesserung infolge transkranieller Hirnstimulation und Infrarotlaser-Stimulation die kognitive Lernleistung tatsächlich verbessert wurde.

Im Gegensatz dazu zeigte sich in der Nocebogruppe kein signifikanter Unterschied in der Lernleistung. Dies bedeutet, dass allein der Gedanke, dass eine transkranielle Strom-und Laserstimulation die Lernfähigkeit verschlechtert, keinen signifikanten Einfluss auf die tatsächliche Lernfähig- 
keit zur Folge hat. Somit zeigt sich, dass lediglich Placeboeffekte eine veränderte kognitive Leistung der Probanden während dieses Experimentes zur Folge hatten. Siehe Diagramm 3 für die Lernleistung am ersten Tag und Tag zwei in den Placebo- und Nocebogruppen.

\begin{tabular}{cccc}
\hline \hline & Odd Ratios & $2,5 \%$ & $97,5 \%$ \\
\hline CD Paar & 0,83 & & \\
EF Paar & 0,40 & 0,71 & 0,98 \\
Block 2 & 2,05 & 0,35 & 0,47 \\
Block 3 & 2,02 & 1,75 & 2,42 \\
Block 4 & 2,51 & 1,72 & 2,38 \\
Nocebogruppe & 0,56 & 2,13 & 2,98 \\
\hline \hline
\end{tabular}

Tabelle 5: Potenziert Koeffizienten (Wahrscheinlichkeits-Verhältnisse) und Vertrauensintervalle an Tag Zwei. 


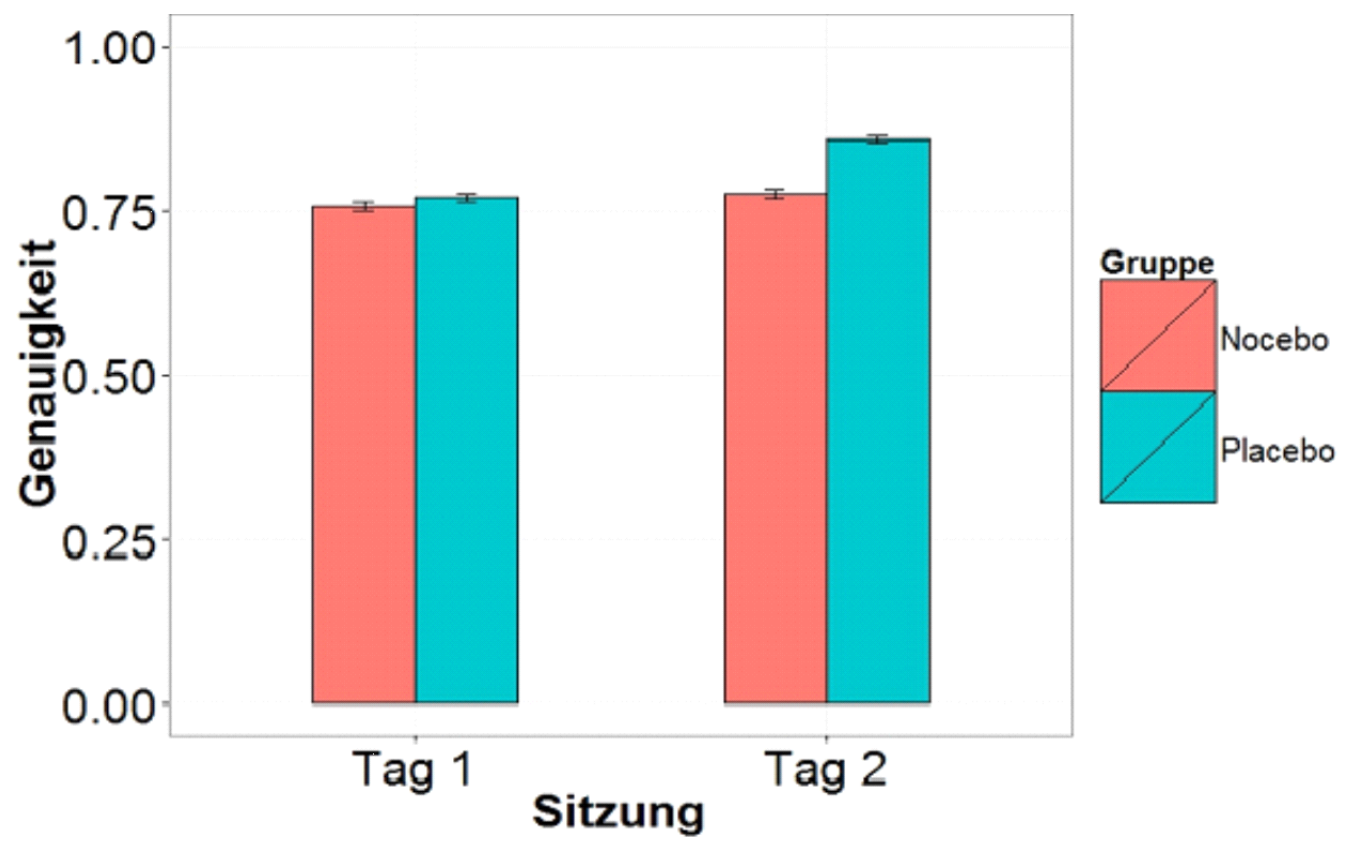

Diagramm 1. Lernleistung an Tag eins und Tag zwei in beiden Gruppen. Die Balken repräsentieren die Standardfehler des Mittelwertes. In der Nocebogruppe zeigt sich kein signifikanter Unterschied in der Lernleistung von Tag eins zu Tag zwei. In der Placebogruppe hingegen zeigt sich am zweiten Versuchstag eine signifikante Verbesserung der Lernleistung.

\subsection{Der Zusammenhang zwischen Persönlichkeitseigenschaften und Place- bo-/ Noceboeffekten}

$B I S / B A S$

Es wurde untersucht, ob Persönlichkeitsmerkmale mit der Stärke der Placebobeziehungsweise Noceboeffekte korrelieren. Im ersten Schritt wurde die allgemeine Lernfähigkeit für jeden einzelnen Probanden in der Placebo- und Nocebogruppe für Tag eins und Tag zwei getrennt berechnet. Im Anschluss wurden die Ergebnisse von Tag eins von denen von Tag zwei subtrahiert. Während positive Werte bedeuten, dass die kognitive Lernleistung der Teilnehmer sich vom ersten zum zweiten Tag verbesserte, zeigt ein negativer Wert das Gegenteil an. Nach Auswertung des BIS/BAS- Fragebogens, den jeder Proband der Placebogruppe vor dem Experiment ausfüllte, wurden die Werte für die BAS-bezogenen Skalen (BAS Drive, BAS Fun search und BAS Reward Responsiveness) und für die BIS-bezogenen Skala (BIS) eingerichtet. Die lineare Regression zeigt keinen signifikanten Effekt der Persönlichkeitsmerkmale auf die Stärke von Placeboeffekten in der Placebogruppe 
(alle ps $>0,166$ ). Hieraus wird geschlussfolgert, dass in unserer Studie kein Zusammenhang zwischen Placeboeffekten und Persönlichkeitsmerkmalen in der Placebogruppe detektiert werden konnten.

\section{PLACEBOGRUPPE}
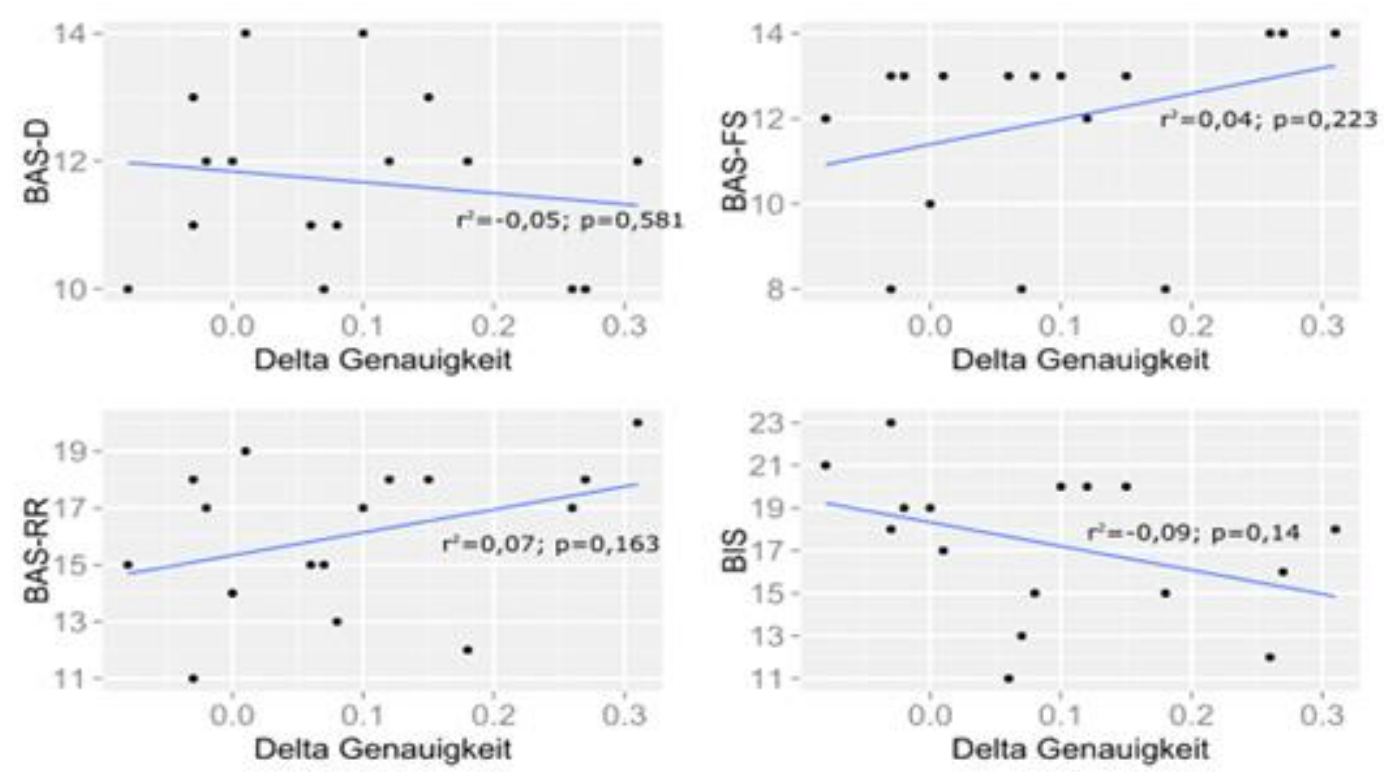

Diagramm 2. Auswertung des BIS/BAS- Fragebogens; lineare Regression in der Placebogruppe; kein signifikanter Effekt der Persönlichkeitsmerkmale auf die Stärke der Placeboeffekte (alle ps> 0,166). 
Analog der Placebogruppe wurden die BIS/ BAS- Probandenfragebögen der Nocebogruppe ausgewertet. Die lineare Regression zeigt keine signifikanten Auffälligkeiten der Persönlichkeitsmerkmale in der Nocebogruppe (alle ps> 0,137). Es sei noch einmal darauf hingewiesen, dass in unserer Studie generell keine signifikanten Noceboeffekte ermittelt werden konnten. Es konnte demnach kein Zusammenhang zwischen Persönlichkeitsmerkmalen und der Stärke von Noceboeffekten in der Nocebogruppe ermittelt werden.

\section{NOCEBOGRUPPE}
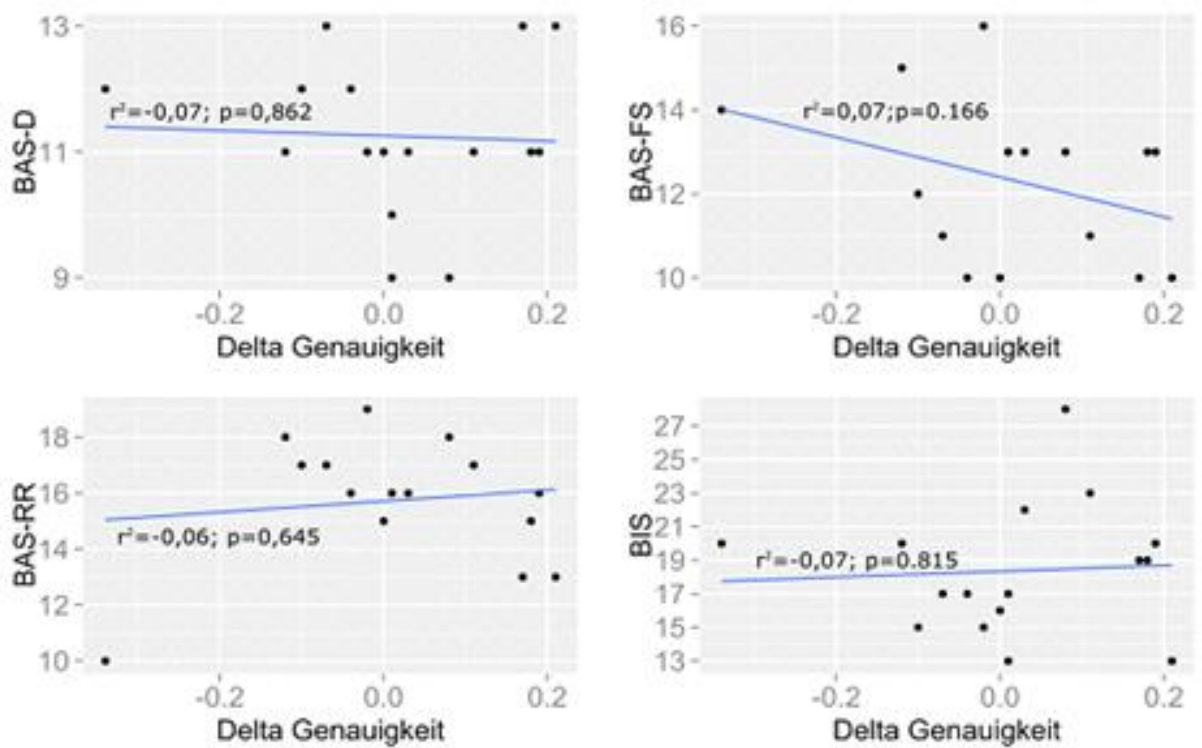

Diagramm 3. Auswertung des BIS/BAS- Fragebogens; lineare Regression in der Nocebogruppe; kein signifikanter Effekt der Persönlichkeitsmerkmale auf die Stärke der Noceboeffekte (alle ps>0,137).

LOT-R

Ähnlich wie bei BIS/BAS wurde eine lineare Regression auf der allgemeinen LOT- R Skala durchlaufen. Die lineare Regression zeigte keinen signifikanten Effekt- weder für die Placebo- $(\mathrm{r} 2=$ $-0,071 ; \mathrm{p}=0,853)$ noch für die Nocebogruppe $(\mathrm{r} 2=-0,069 ; \mathrm{p}=0,959)$. Somit konnte auch in dieser Analyse kein Zusammenhang zwischen Persönlichkeitseigenschaften und der Stärke der Placeboeffekte in der Placebogruppe sowie Noceboeffekten in der Nocebogruppe ermittelt werden. 


\subsection{Die Bedeutung der Händigkeit in Bezug auf Placebo-und Noceboeffekte}

Der mittlere Wert für die Händigkeit der Probanden lag bei 63,41 \pm 49,77 (SD). Es wurde festgestellt, dass die Händigkeit keinen Einfluss auf Placebo- und Noceboeffekte hatte. 


\section{Diskussion}

Zahlreiche Studien der vergangenen Jahre detektierten bereits einen Zusammenhang zwischen der Instruktion, die den Probanden gegeben wird und der daraus resultierenden Erwartungshaltung (z.B. Finniss et al. 2010; Klosterhalfen und Enck 2006; Benedetti et al. 2005).

Die vorliegende Studie untersuchte den Einfluss von verbalen Placebo-und Noceboeffekten auf kognitive Funktionen wie das instrumentelle Lernen. Hierbei wurde erkannt, dass eine PlaceboHirnstimulation die kognitiven Funktionen signifikant verbessern konnte. Durch verbale Manipulation konnte die Erwartungshaltung der Probanden positiv beeinflusst werden. Eine Nocebostimulation hingegen führte in unserem Experiment zu keiner Veränderung der kognitiven Lernfähigkeit.

Zur Ergebnisfindung wurde eine Standardaufgabe für implizites Lernen verwendet. Es gibt bisher nur wenige weitere Studien, die sich mit der Beeinflussung von kognitiven Funktionen durch Placeboeffekte auseinander gesetzt haben. Die zahlreichen Studien zur Wirkung von Placebos auf den Menschen beschäftigen sich zum aktuellen Zeitpunkt hauptsächlich mit der Beeinflussung von Schmerzen und Depressionen (Peciña et al. 2013; Häuser et al. 2012a; Schweinhardt et al. 2009; Rief et al. 2009a; Kaptchuk et al. 2006; Brown 1994). Uns ist es jedoch mit der aktuellen Studie gelungen, lediglich durch eine manipulierte Erwartungshaltung der Probanden eine Veränderung der kognitiven Lernleistungen zu evozieren. Die Erwartungshaltung wurde manipuliert, in dem man den Probanden vor der Lösung der Aufgaben erklärte, dass sie im Folgenden eine Hirnstimulation in Form von tDCS und Infrarotlaserstimulation erhalten würden. Der Placebogruppe erklärte man, dass diese Stimulation die Lernfähigkeiten für eine begrenzte Zeit verbessern würde. Den Teilnehmern der Nocebogruppe erklärte man in der Vorinformation das genaue Gegenteil. Die folgende Stimulation würde die Lernleistung zeitlich begrenzt verringern. Diese Erwartungshaltungen wurden noch verstärkt, in dem auch die Rückmeldungen der Aufgaben manipuliert wurden. In der zweiten Lernphase der Placebogruppe war die Aufgabenstellung deutlich vereinfacht, somit gingen die Probanden davon aus, dass die Stimulation bereits ihre kognitiven Leistungen verbessert hat. Auch in der Nocebogruppe wurden manipulierte Rückmeldungen eingesetzt.

Durch die deutlich erschwerte Aufgabenstellung im Verhältnis zur ersten Lernphase wurde das Gefühl der Probanden noch mehr verstärkt, dass die Stimulation tatsächlich die kognitiven Leistungen verringerte. 


\subsection{Experimentelle und klinische Placebostudien zur Beeinflussung von kognitiven Funktionen}

Zum aktuellen Zeitpunkt gibt es neben der hier vorgestellten Studie nur wenige weitere klinische Studien, die sich mit der Thematik der Beeinflussung der kognitiven Funktionen durch Placeboeffekte beschäftigen. Sie sollen im Folgenden kurz vorgestellt und analysiert werden.

Eine Studie zur entsprechenden Thematik stammt aus dem Jahr 2009. Den 31 Probanden wurde im Vorfeld erklärt, dass sie an einem Experiment teilnehmen, in dem eine Koffeintablette zur kognitiven Leistungssteigerung getestet würde. Hierzu wurden zwei Experimente durchgeführt. In beiden Teilexperimenten erhielten die Probanden inkorrekte Rückmeldungen über ihre kognitive Leistungsfähigkeit. Die Teilnehmer der Verumgruppe bekamen falsch positive Rückmeldungen, die Placebogruppe eher negative Rückmeldungen. Somit wurde bewusst die Vermutung der Teilnehmer beeinflusst, ob sie der Placebogruppe oder der Verumgruppe zugeteilt wurden. Allein diese Vermutung scheint Einfluss auf die kognitive Leistung ausgelöst zu haben, denn die Teilnehmer, die aufgrund der positiven Rückmeldungen glaubten, dass sie die aktive Behandlung bekamen, hatten eine höhere Genauigkeit in Experiment eins und schnellere Reaktionszeiten in Experiment zwei als diejenigen, die glaubten, dass ihnen ein Placebo verabreicht worden war. Die Einbeziehung der Placebo- Kontrollgruppe in Experiment zwei zeigte, dass diese Effekte nicht nur durch die Rückmeldungsmanipulation selbst erklärt werden konnten, sondern eine verbesserte Leistung auch durch die Erwartungshaltung der Teilnehmer ausgelöst wurde. Diese Ergebnisse lassen einen Rückschluss auf die Wichtigkeit der Teilnehmerüberzeugungen über ihre Behandlungszuteilung in echten doppelblinden Studien zu. Teilnehmer scheinen schlechtere Ergebnisse zu erzielen, wenn sie wissen oder auch nur vermuten, dass sie der Placebogruppe angehören (Colagiuri und Boakes 2010).

Vergleicht man diese Studie mit der aktuell vorgestellten Studie sind einige Parallelen, aber auch Unterschiede feststellbar. In beiden Fällen hatte eine positive Erwartungshaltung der Probanden eine positive Beeinflussung der kognitiven Lernfähigkeit zur Folge. Im Folgenden sollen die Unterschiede aufgeschlüsselt werden.

Bei nahezu identischer Teilnehmerzahl waren in der Studie von Colagiuri und Boakes sowohl männliche als auch weibliche Versuchsteilnehmer zugelassen. Einmal wurde eine Placebopille verabreicht, in unserem Fall eine Placebo- Hirnstimulation. Eine weitere Differenz stellt dar, dass bei Colagiuri und Boakes die Teilnehmer wussten, dass es eine Placebo- und eine Verumgruppe gibt. Durch manipuliertes Feedback nach den Aufgabenblöcken wurden ihre Vermutungen bewusst in die eine oder andere Gruppe gelenkt. Sie sollten später erläutern, welche Vermutung sie bezüglich der 
Gruppe hatten.

In der aktuellen Studie bekamen zwar alle Probanden eine Placebostimulation, jedoch gingen alle davon aus, dass sie eine Verumstimulation erhielten. Somit waren alle Teilnehmer unvoreingenommen, weil sie die Placebostimulation nicht vermuteten. Es konnte bewiesen werden, dass eine positive Erwartungshaltung, ausgelöst durch die manipulierten Vorinformationen so stark war, dass Placeboeffekte zur tatsächlichen Steigerung der Leistung führten. Im Beispiel von Colagiuri und Boakes war eine Leistungsverbesserung nur für den Fall zu verzeichnen, in dem die Probanden glaubten, nicht der Placebo- sondern der Verumgruppe anzugehören. Der Gedanke daran, dass die Probanden der Placebogruppe angehörten, verringerte die Erwartungshaltung an den Erfolg der Koffeinpille und führte somit zu schlechteren Ergebnissen.

Zusammenfassend kann festgestellt werden, dass es von enormer Wichtigkeit für die korrekte Ergebnisfindung in doppelblinden placebokontrollierten Studien ist, dass die Probanden im Unklaren darüber sein müssen, ob sie der Placebo- oder Verumgruppe angehören.

Eine weitere Studie von Colagiuri et al. beschäftigte sich ebenfalls mit der Frage, ob Erwartungen Placeboeffekte in Bezug auf das implizite Lernen auslösen können. Hierzu wurde jedoch ein bedeutend größeres Probandenkollektiv von 464 Teilnehmern untersucht. Zur Ergebnisfindung führte man das folgende Experiment durch: Die Probanden lösten am PC visuelle Suchaufgaben. Währenddessen konnten sie manchmal einen Geruch wahrnehmen, manchmal nicht. Vorab erhielten die Teilnehmer positive, negative oder keine Informationen über die Wirkung der Gerüche auf das implizite Lernen, je nach Gruppe. Die Probanden, die im Vorfeld erfuhren, dass der Geruch während der Aufgaben eine gesteigerte Lernfähigkeit zur Folge hat, zeigten im Nachhinein tatsächlich schnellere Reaktionszeiten. Die Teilnehmer, die im Vorfeld erfuhren, dass die Gerüche während der Aufgaben die Lernleistung beeinträchtigen würden, zeigten langsamere Reaktionszeiten als die Teilnehmer, die keine Vorinformationen über die Gerüche erhielten. Somit konnte ermittelt werden, dass bewusste Lehrmanipulationen Placeboeffekte auf die Lernfähigkeit der Teilnehmer evozieren können.

Analog unserer Studie konnten positive Vorinformationen in den Probanden eine positive Erwartungshaltung auslösen und somit durch einen Placeboeffekt eine Lernleistungsverbesserung evozieren. Jedoch konnten Colagiuri et al. auch durch negative Vorinformationen eine Verschlechterung der Lernleistung und somit einen Noceboeffekt provozieren, was in der aktuell vorgestellten Studie nicht gelang. Hierbei muss jedoch auch die deutlich höhere Probandenzahl beachtet werden (Colagiuri et al. 2011). Außerdem ist hier noch einmal auf die Studie von Ströhle aus dem Jahr 2000 hinzuweisen. Dieser fand heraus, dass besonders bei Frauen Noceboeffekte ausgelöst 
werden können. Bei Colagiuri et al. bestand das Probandenkollektiv aus 66\% weiblichen Teilnehmern, unsere Studie jedoch bestand lediglich aus männlichen Teilnehmern. Somit lässt sich anhand unserer Ergebnisse erneut der Verdacht äußern, dass Frauen eher zu Noceboeffekten neigen als Männer.

Eine neuere Studie aus dem Jahr 2013 beschäftigte sich mit der Beeinflussung von kognitiven Funktionen und motorischen Funktionen durch Placeboeffekte. In diesem Fall handelte es sich bei den Probanden jedoch um Parkinsonpatienten mit tiefer Hirnstimulation und hyperkinetisch starren Subtyp. Es ist bekannt, dass tiefe Hirnstimulation (STN-DBS) zwar motorische Bewegungsabläufe bei Parkinsonpatienten verbessert, jedoch kognitive Funktionen wie zum Beispiel die Sprachflüssigkeit beeinträchtigen kann. Bei dem Experiment, an dem 24 Patienten teilnahmen, wurde die Erwartung an die tiefe Hirnstimulation im Vorfeld bewusst manipuliert. Es gab eine Placebogruppe, welche nur positive Informationen über die Wirkung der tiefen Hirnstimulation erhielt, eine Nocebogruppe mit negativen Vorinformationen und eine Kontrollgruppe. Der letzteren wurden lediglich allgemeine neutrale Vorinformationen gegeben. In der Placebogruppe erklärte man den Patienten, dass die folgende Stimulation die motorischen Funktionen stark verbessern würde, der Nocebogruppe erklärte man das genaue Gegenteil. Der neutralen Kontrollgruppe wurde erklärt, dass die Stimulation keinen Einfluss auf die motorischen Funktionen zeigen wird. Alle Teilnehmer bekamen jedoch eine identische Stimulation. Entsprechend der Gruppe erhielten die Patienten einmal ihre normalen Antiparkinsonmedikamente, am zweiten Versuchstag des jeweiligen Teilnehmers durften diese nicht eingenommen werden. Somit entstanden insgesamt sechs unterschiedliche Probandengruppen. Die Bewegungsfunktionen der einzelnen Teilnehmer wurden zuvor mit einer entsprechenden Skala gemessen. Bei den Patienten ohne Antiparkinsonmedikation konnten durch die manipulierte Erwartungshaltung in der Placebogruppe die proximalen, jedoch nicht die distalen Bewegungsabläufe signifikant im Vergleich zur Nocebo- und Kontrollgruppe verbessert werden. Die Teilnehmer zeigten außerdem eine Beeinträchtigung der verbalen Fähigkeiten. Somit konnte gezeigt werden, dass eine manipulierte positive Erwartungshaltung der Parkinsonpatienten zur tiefen Hirnstimulation einerseits einen Placeboeffekt auf die motorischen Funktionen auslöst, zum anderen sogar einen Noceboeffekt, welcher zu einer Verschlechterung der verbalen Fähigkeiten führte. Obwohl alle Probanden eine identische Stimulation erhielten, zeigten sich lediglich in der Placebogruppe signifikante Ergebnisse (Keitel et al. 2013a).

Vergleicht man diese Ergebnisse mit denen aus unserer Studie, ist erkennbar, dass auch in diesem Beispiel eine positive Erwartungshaltung der Probanden einen bedeutend stärkeren Placeboeffekt evozieren konnte als eine negative Erwartungshaltung. In der Studie von Keitel et al. 
wurden die Erwartungshaltungen ebenfalls durch manipulierte Probandeninformationen ausgelöst. Es sei jedoch zu erwähnen, dass Keitel et al. mit Verumstimulation stimuliert haben. Somit wurden die zu erwartenden Therapieeffekte lediglich verstärkt. In unserer Studie jedoch wurde ausschließlich mit einer Sham-Stimulation stimuliert. Es lässt sich somit erkennen, dass unsere verbesserten Lernerfolge auf reine Placeboeffekte zurückzuführen sind. Diese Ergebnisse stellen einen sehr interessanten Anhaltspunkt für künftige wissenschaftliche Studien dar.

Keitel et al. führten 2013 eine weitere Studie mit Parkinsonpatienten durch. Der Versuchsaufbau war analog der zuvor vorgestellten Studie, jedoch wurde in diesem Fall das Augenmerk auf ein weiteres Leitsymptom von Parkinson, den rezidivierenden Tremor, gelegt. Auch hierbei gab es eine Placebogruppe, eine Nocebogruppe und eine Kontrollgruppe. Erneut erhielt die Placebogruppe positive Vorinformationen über die folgende Hirnstimulation. Die Stimulation würde den Tremor deutlich reduzieren. Die Nocebogruppe erhielt negative Vorinformationen. Durch die Hirnstimulation könne sich der Tremor noch verstärken. Somit konnten erneut die Erwartungen der Probanden an die Stimulation manipuliert werden. Bei den Probanden mit positiver Erwartungshaltung verbesserte sich der Tremor um bis zu 10\% im Vergleich zur Kontrollgruppe, die Probanden mit negativer Erwartungshaltung erlebten eine Verschlechterung des Tremors im Vergleich zur Kontrollgruppe, welche lediglich neutrale Informationen über die Studie erhielt. Außerdem zeigte sich in der Nocebogruppe mit Antiparkinsonmedikation eine Verschlechterung der Sprachflüssigkeit im Vergleich zu den anderen Gruppen. Eine verschlechterte verbale Fähigkeit ist ein bekannter Nebeneffekt der STN- DBS (Keitel et al 2013b). Erneut konnte, wie auch in unserer Studie, die enorme Wichtigkeit der Erwartungshaltung der Patienten in Bezug auf eine erfolgreiche Therapie bewiesen werden. Allein positive Vorinformationen zu einer bestimmten Therapie können die normalerweise eintretenden Erfolge um ein Vielfaches steigern. Somit kann die positive Erwartungshaltung der Patienten zur bevorstehenden Therapie eine Steigerung des Behandlungserfolges bewirken. Im Gegensatz dazu wurde in der Studie von Keitel et al. erkannt, dass Noceboeffekte, ausgelöst durch eine negative Erwartungshaltung der Patienten zur bevorstehenden Therapie, den Therapieerfolg verringern können. In unserer Studie jedoch lösten Noceboeffekte keine Verschlechterung der Leistungen aus.

Nun stellt sich die Frage, wie diese Differenzen entstehen können. Dazu entwickelte ich drei Lösungsansätze: Zum einen waren die Probanden in unserer Studie vollkommen gesund. Eventuell kann man bei Gesunden negative Erwartungshaltungen durch manipulierte Vorinformationen zur bevorstehenden Behandlung nur in geringem Maße evozieren. Bei den Probanden in unserer Studie ging es lediglich um eine Lernleistungsverschlechterung, die laut Vorinformation höchstens 30 min 
anhalten würde. Diese Auswirkung ist irrelevant für das weitere Leben der Probanden. Die Patienten von Keitel et al. dachten jedoch in der Nocebogruppe, dass sie eine Stimulation erhielten, die Ihren Tremor noch verschlechtern würde. Dies könnte in ihnen Ängste und somit Noceboeffekte ausgelöst haben, dass sich ihre Lebensqualität noch weiter verschlechtern könnte.

Die zweite Erklärung, dass in unserem Experiment im Gegensatz zu Keitel et al. in der Nocebogruppe keine Verschlechterung der Leistung auftrat, geht in die Richtung, dass wir sowohl die Probanden der Placebo- als auch der Nocebogruppe mit einer Sham- Stimulation stimuliert haben. Bei Keitel et al. wurde mit Verum- STN-DBS stimuliert.

Als dritte mögliche Ursache sei erneut darauf hingewiesen, dass in unserer Studie nur männliche Probanden teilnahmen. Außerdem sei noch einmal auf die Studie von Rosenzweig et al. 1993 hingewiesen. Sie erkannten, dass eher ältere Menschen zur Ausbildung von Noceboeffekten neigen. Die Probanden in der Studie von Keitel waren im Durchschnitt älter als 60 Jahre. Das Durchschnittalter unserer Probanden lag bei 24,66 \pm 2,54 Jahren.

\subsection{Beispiele aus klinischen Placebo-/Nocebostudien}

Zum Thema Placebo- und Nocebophänomen ist besonders in den vergangenen Jahren ein vermehrtes wissenschaftliches Interesse zu verzeichnen, da diese Phänomene durchaus spannend sind aber dennoch zum aktuellen Zeitpunkt nicht vollständig durchleuchtet werden konnten. Unsere Studie machte es sich zur Aufgabe, Placebo-/ Noceboeffekte im Zusammenhang mit instrumentellen Lernvorgängen nachzuweisen. Hierzu wurde eine Erwartungshaltung durch verbale Manipulationen ausgelöst.

Es ist bekannt, dass nicht nur Placebos in Tablettenform eine Wirkung auf den Menschen auslösen können. Die folgende Studie beweist, dass selbst eine Scheinoperation den Gesundheitszustand der Probanden deutlich verbessern konnte. Dimond et al. berichten 1960 in einer Studie über zwei Gruppen von Angina pectoris- Patienten. Die eine Gruppe erhielt einen wirkungsvollen chirurgischen Eingriff, die zweite Gruppe hingegen wurde nur scheinoperiert. Erstaunlicherweise erlebten alle Patienten eine Besserung nach dem Eingriff (Dimond et al. 1960).

Ein weiteres Beispiel einer wirkungsvollen Scheinoperation führte der Chirurg Bruce Moseley durch. Er ist ein bekannter Spezialist für Gelenkerkrankungen. Er bildete unter seinen KniearthrosePatienten zwei Gruppen. Die eine Gruppe erhielt den operativen Eingriff, der zweiten Gruppe wurde lediglich ein Hautschnitt gesetzt und dieser im Anschluss vernäht. Alle Patienten gingen davon aus, tatsächlich operiert worden zu sein. Erstaunlicherweise waren die scheinoperierten Patienten 
genauso zufrieden mit dem Behandlungsergebnis wie die tatsächlich operierten. Neben einem bewiesenen Placeboeffekt kann jedoch auch der Aspekt betrachtet werden, dass Kniegelenksoperationen häufig nicht notwendig sind, weil die Beschwerden auch von selbst bzw. durch Schonung verschwinden können (Moseley et al. 2002).

2009 konnten Linde et al. den Placeboeffekt auch bei Akupunktur nachweisen. Dazu wurden Daten aus 33 Studien zur Wirkung von Akupunktur auf Spannungskopfschmerzen und Migräne verglichen. Es zeigte sich, dass sowohl die wirkliche Akupunktur als auch eine Schein- Akupunktur einen Behandlungserfolg bewirkten. Beim Spannungskopfschmerz wurden bessere Ergebnisse durch die wirkliche Akupunktur erzielt. Interessanterweise wirkte jedoch die Scheinakupunktur bei Migräne besser als die reelle (Linde et al. 2009). Vermutlich besitzt Akupunktur bei Migräne nur einen geringeren Wirkungsgrad. Menschen mit Migräne sind häufig sehr verzweifelt und im täglichen Leben eingeschränkt. Die Erwartungshaltung, dass nun endlich eine neue Methode die Migränesymptome lindern könnte, scheint so stark zu sein, dass die Symptome tatsächlich verbessert wurden. Hierbei handelt es sich um ein weiteres interessantes Einsatzgebiet von Placebos in der Medizin.

Neben zahlreichen Placebostudien existiert auch eine geringe Anzahl an klinischen Nocebostudien. Diese sind seltener da sie teilweise ethisch nicht zu vereinbaren wären. Eine kleine Auswahl soll im Folgenden vorgestellt werden. In einer 1990 durchgeführten Studie zeigten 25\% aller Patienten mit Nahrungsmittelallergie allergische Reaktionen, obwohl sie anstatt dem beschriebenen Allergen lediglich Kochsalzlösung injiziert bekamen. Hierbei handelt es sich um ein deutliches Nocebophänomen (Jewett et al. 1990). Eine 2004 durchgeführte Studie zu unerwünschten Nebenwirkungen von oral verabreichten Arzneimitteln kam zu dem Ergebnis, dass 27\% der Probanden aus der Placebogruppe Nebenwirkungen erlebten. Alle Probanden wurden zuvor ausführlich über mögliche unerwünschte Nebenwirkungen aufgeklärt (Liccardi et al. 2004). In einer ähnlichen Studie aus dem Jahr 2008 empfanden lediglich 3\% der Probanden des Placebokollektives die zuvor beschriebenen möglichen Nebenwirkungen (Lombardi et al. 2008).

In den frühen 1980er Jahren wurde mit 34 Versuchsteilnehmern folgendes Experiment durchgeführt: Einem Probandenkollektiv, bestehend aus Studenten, wurde gesagt, dass sie einen elektrischen Strom durch den Kopf erhalten würden. Dabei können Kopfschmerzen auftreten. Ohne tatsächlichen Stromfluss klagten mehr als zwei Drittel der Versuchsteilnehmer über Kopfschmerzen (Reid 2002).

Dass eine Placebobehandlung subjektive Symptome von Patienten signifikant verbessern kann, konnte bereits in zahlreichen Studien nachgewiesen werden (Dimond et al. 1960; Moseley et al. 
2002; Linde et al. 2009; Rief et al. 2009a; Häuser et al. 2009a; Kaptchuk et al. 2006). Allerdings wird weithin angenommen, dass die Reaktion auf eine Placebobehandlung eine Täuschung des Patienten erfordert. Dieser Aspekt der Placebobehandlung stößt teilweise an seine ethischen Grenzen.

Eine Studie aus dem Jahr 1965 lässt erkennen, wie stark die Reaktion auf ein Placebo vom Vertrauen zum Behandler abhängt. Psychoneurotische Patienten bekamen ein Placebo in Tablettenform mit dem ausdrücklichen Hinweis des Arztes, dass es sich um „Zuckerpillen“ ohne jeglichen Wirkstoff handele. Die Worte des Arztes lauteten: "Vielen Leuten mit ihrer Erkrankung wurde mit etwas geholfen, was man manchmal Zuckerpillen nennt, und wir glauben, dass auch Ihnen eine sogenannte Zuckerpille helfen könnte. Wissen Sie, was eine Zuckerpille ist? Eine Zuckerpille ist eine Pille, in der überhaupt kein Medikament drin ist. Ich denke, diese Pille wird Ihnen helfen, wie sie schon so vielen anderen geholfen hat. Wollen Sie diese Pille ausprobieren?" Die Patienten bekamen den Auftrag, diese Pillen eine Woche lang dreimal am Tag einzunehmen. Nach dieser Woche würden sie eine endgültige Behandlungsempfehlung erhalten. Vierzehn Patienten stellten sich nach der Woche wieder bei dem Arzt vor und berichteten über Verbesserungen. Die Verbesserungen traten auf, weil die Patienten durch das Vertrauen in den Arzt davon überzeugt waren, dass er ihnen mit der Behandlung tatsächlich helfen möchte. Der Placeboeffekt könnte jedoch auch in der versprochenen speziellen Behandlung, welche nach einer Woche folgen sollte, begründet liegen (Park und Covi 1965). Es sei jedoch angemerkt, dass das Patientenkollektiv lediglich aus 15 Teilnehmern bestand und die Erfolge auch auf eine Spontanremission zurückzuführen sein können.

Eine jüngere Studie mit ähnlichem Inhalt, jedoch einem größeren Probandenkollektiv stammt aus dem Jahr 2010. Kaptchuk et. al. 2010 haben untersucht, ob eine nicht verdeckte Placeboabreichung (Open-Label- Placebo) ebenfalls Verbesserungen der Symptome auslösen kann. Es wurde eine Studie mit 80 Reizdarmsyndrompatienten durchgeführt. Die Teilnehmer wurden in zwei Gruppen eingeteilt (Open-Label- Placebo und No- Treatment Kontrollgruppe). Kaptchuk behandelte die Patienten mit Pillen, zu denen er ihnen sagte, es handele sich um Placebos ohne aktive Inhaltsstoffe, eine Art Zuckerpillen. Kaptchuk erklärte den Teilnehmern aber auch, dass diese Pillen bereits in klinischen Studien zu einer signifikanten Besserung der Reizdarmsymptome geführt hatten. In dieser kleinen Studie konnte bewiesen werden, dass auch eine offene Placebogabe signifikante Verbesserungen in der Symptomatik des Reizdarmsydroms bewirken kann. Diese herausragende Entdeckung sollte der Grundstein für weitere Untersuchungen sein.

Die Ergebnisse von Kaptchuk et al. 2010 konnten später in einer kleinen Studie zur Behandlung einer Major Depression bestätigt werden. Hierbei zeigte sich ein positiver Effekt der offenen Placebogabe auf depressive Symptome. Dieser Effekt wurde auch von den Teilnehmern der 
Studie bestätigt (Kelley et al. 2012).

In einer weiteren Überprüfung des Placeboeffektes bei Migränepatienten konnten Wissenschaftler zeigen, dass eine offene Placebogabe einer No- Treatment-Bedingung überlegen war. Das Verumpräparat war ähnlich stark wirksam wie ein Placebo, wenn es auch als solches deklariert war, wie ein Placebo, welches als Medikament ausgegeben wurde (Kam- Hansen et al. 2014).

\subsection{Erkennung der Placebostimulation}

In der hier vorliegenden Arbeit erkannten weder die Versuchsleiterin noch die Probanden, dass es sich um keine echte Stimulation handelte. Auch der Neurologe, der die Voruntersuchung durchführte, wurde zuvor nicht über den tatsächlichen Inhalt der Studie in Kenntnis gesetzt. Somit lag das Design einer Doppelblindstudie vor.

Eine Analyse von Fertonani et al. mit 693 verschiedenen tES- Sitzungen kam zu dem Ergebnis, dass tRNS und tACS weniger starke Hautempfindungen im Vergleich zur tDCS auslösen. Dieser Effekt war besonders bei anodaler tDCS verstärkt. Außerdem lösten größere Elektroden stärkere Hautempfindungen aus. Höhere Intensitäten der Stromstärke wurden ebenfalls stärker wahrgenommen. Der Zeitpunkt der Stimulation und die Anbringung der Elektroden an unterschiedlichen Arealen hatten hingegen keinen Einfluss auf die Stärke der empfundenen Hautbeschwerden. Die Hautempfindungen werden durch Hautrezeptoren des somatosensomotorischen Systems ausgelöst. Die Analyse ergab weiterhin, dass sich aufgrund der geringeren Hauteffekte eine tES weniger gut zur Sham- Stimulation eignet. Eine bessere Variante bei Sham- Stimulationen stellt die anodale tDCS dar (Fertonani et al. 2015).

In unserer Studie wurden alle Probanden der Placebo- und Nocebogruppe mit einer ShamtDCS- und fake- Infrarotlaserstimulation stimuliert. In den meisten anderen Studien hingegen erhalten die Probanden eine reelle tDCS und lediglich in der Kontrollgruppe eine Sham- Stimulation. Während einer Sham- Stimulation können die Probanden Hautempfindungen wie Kribbeln, Juckreiz oder Brennen wahrnehmen. Dies ist sehr wünschenswert, damit alle Probanden von einer aktiven tDCS ausgehen konnten. Das Kribbeln lässt sich erklären, da sich die einminütige Sham-Stimulation in 20s ,ramp up‘, 30s $1 \mathrm{~mA}$ Stimulation und 10s, ramp down' gliederte. Dies bedeutet, dass sich für 20s ein Gleichstrom aufbaut, dieser 30s konstant gehalten wird und in den nächsten 10s wieder abgebaut wird. Im Gegensatz zur reellen tDCS hat die Sham- Stimulation jedoch keinen Einfluss auf die kortikale Exzitabilität (Nitsche und Paulus 2007).

Die Probanden der aktuell vorgestellten Studie wurden in der Stimulationsinformation über mögliche Nebenwirkungen einer Verum- tDCS aufgeklärt, da sie keinen Verdacht schöpfen durften, 
dass sie lediglich eine Sham- Stimulation bekommen würden. Alle Probanden der hier erläuterten Studie empfanden Hautempfindungen während der Stimulation. Nahezu alle Probanden beschrieben ein leichtes Kribbeln unter den Elektroden. Dieser Effekt war durchaus erwünscht, damit die Probanden von einer aktiven Stimulation ausgehen konnten. Zwei Probanden beschrieben ein leichtes Brennen unter den Elektroden. Kein Teilnehmer klagte über Kopfschmerzen, Schlafstörungen oder weitere Missempfindungen. Interessanterweise waren die empfundenen Hauterscheinungen in der Nocebogruppe signifikant höher als in der Placebogruppe. Als Ursache der erhöhten Hautempfindungen in der Nocebogruppe vermute ich einen Noceboeffekt, der durch die negative Erwartungshaltung der Patienten in Bezug auf die Stimulation ausgelöst wurde.

\subsection{Der Einfluss der Versuchsleiterin auf die Stärke der Placeboeffekte}

Die bereits in der Einleitung erwähnte Studie von Gliedman et al. aus dem Jahr 1965 ist ein gutes Beispiel, wie wichtig das Vertrauen des Patienten zum Medikamentenverabreicher in Bezug auf den Behandlungserfolg zu sein vermag. Der Arzt stellt in den meisten Fällen eine gehobene Position für einen Patienten dar.

Es ist somit erkennbar, dass ein Zusammenhang zwischen der Sympathie und dem Vertrauen zwischen dem Behandler und dem Patienten in Bezug auf Placeboeffekte besteht. Wenn der Patient den Behandler als besonders vertrauenswürdig bewertet, können verstärkte Placeboeffekte auftreten. Einen Schlüssel zur erfolgreichen Arzt- Patienten- Kommunikation und somit zum Vertrauensaufbau stellt die Empathie dar. Empathie bedeutet, dass der Behandler dem Patienten zuhört, ihn aussprechen lässt und versucht, sich in die Lage des Patienten hineinzuversetzen. Nur wenn sich der Behandler Zeit für die Sorgen und Ängste der Patienten nimmt, kann eine Vertrauensbasis aufgebaut werden.

Weibliche Behandler neigen eher dazu, sich ausgiebiger mit den Patienten zu unterhalten und unterbrechen das Gespräch weniger. Des Weiteren übermitteln sie häufiger Zeichen von nonverbaler Kommunikation. Daraus könnten durchaus größere Placebowirkungen resultieren (Kentenich und Pietzner 2012). Da es sich in der aktuell vorgestellten Studie um eine weibliche Versuchsleiterin handelte, könnte darin eine erste Erklärung für die durchaus erfolgreiche Placebostimulation liegen. Die Versuchsleiterin hat die Aufgabenstellungen noch einmal mit eigenen Worten wiederholt und aufkommende Fragen ausführlich erklärt, sodass der Proband erste Unsicherheiten überwinden konnte. Auch die Tastatur, mit der die Aufgaben ausgeführt wurden, erklärte die Versuchsleiterin ausführlich und durchlief selbst einen Probedurchgang. Des Weiteren begleitete die Versuchsleiterin alle Probanden zur neurologischen Voruntersuchung, wartete vor der Tür des Untersuchungsraumes 
und brachte sie anschließend zurück in den Versuchsraum. Somit konnten die Probanden eine Vertrauensbasis aufbauen. Außerdem wurden das Erscheinungsbild und das Verhalten der Versuchsleiterin neutral gewählt, sodass die Probanden keiner Ablenkung ausgesetzt wurden. Bewusst wurde ein weißer Kittel vermieden, um im Probanden keine Ängste auszulösen. Es konnte in früheren Studien gezeigt werden, dass auch die Einstellung des Behandlers gegenüber der Therapie mit dem Behandlungserfolg korrelieren kann. Ist die Einstellung des Arztes positiv, kann dies auch zu einer deutlichen Verbesserung der Symptome führen (Di Blasi et al. 2001).

Eine Metaanalyse von Mora et al. 2011 untersuchte 96 placebokontrollierte, randomisierte Studien zu Antidepressiva. In dieser Zusammenfassung konnten sowohl starke Placeboeffekte in der Placebo- Kontrollgruppe, als auch Noceboeffekte in Form von Nebenwirkungen auf eine inerte Substanz verzeichnet werden. Hierbei sei kritisch angemerkt, dass vermeintliche Placeboeffekte auch im natürlichen Krankheitsverlauf einer Depression begründet liegen können. Es wurde festgestellt, dass Placeboeffekte von den objektiven Beobachtern (Versuchsleitern) deutlich stärker beschrieben werden, als in der Selbsteinschätzung der Probanden. Da die Effektgrößen der Beobachterbewertungen einen signifikanten Anstieg mit dem Publikationsjahr der jeweiligen Studien zeigten, lässt dies auf einen starken Einfluss der Erwartungen der Versuchsleiter auf die Beurteilung der Placeboeffektstärke schließen. Ein Zusammenhang der Effektstärke und dem Publikationsjahr konnte hingegen in den Selbstbewertungen der Probanden nicht nachgewiesen werden. Während der Placeboeffekt für Frauen und Männer ähnlich zu sein scheint, wurden Geschlechterunterschiede für die Nocebo-Raten gefunden. Rief et al. konnten bereits 2009 in 2 Metaanalysen ähnliche Ergebnisse ermitteln (Rief et al. 2009a; Rief et al. 2009b).

Es wird somit deutlich, dass auch der Versuchsleiter keinen Einblick haben sollte, ob er die inerte Substanz oder das Verum -Präparat verabreicht. Diese Erkenntnisse wurden in der aktuell vorgestellten Studie eingesetzt, um Beeinflussungen der Versuchsleiterin auf die Stärke der Placeboeffekte zu vermeiden. Die Versuchsleiterin ging davon aus, alle Probanden mit einer reellen tDCS- und Infrarotlaserstimulation zu stimulieren. Somit erhielt die Studie das Design einer Doppelblindstudie und gleichzeitig einen bedeutend höheren wissenschaftlichen Wert. 


\subsection{Der Zusammenhang zwischen Persönlichkeitseigenschaften und Place- boeffekten}

Es wurde untersucht, ob Persönlichkeitsmerkmale mit der Stärke von Placebo-/ Noceboeffekten korrelieren. Im Allgemeinen sei davon auszugehen, dass optimistische Menschen besser auf eine Placebogabe reagieren. Bei pessimistisch eingestellten Personen lässt sich zum einen vermuten, dass sie weniger empfänglich für Placeboeffekte, aber durchaus empfänglich für Noceboeffekte sein könnten.

In der aktuellen Studie wurden die entsprechenden Persönlichkeitsmerkmale jedes Probanden zuvor anhand von LOT-R- und BIS/BAS- Fragebögen ermittelt. Hierbei konnten jedoch keine signifikanten Ergebnisse entdeckt werden. Dies bedeutet, dass in unserer Studie kein Zusammenhang zwischen den Charaktereigenschaften der Probanden und der Stärke von Placebo-/Noceboeffekten registriert werden konnte.

Andere Autoren fanden jedoch hinsichtlich der Persönlichkeitsmerkmale signifikante Unterschiede. Bei diesen waren Placeboeffekte unterschiedlich stark ausgeprägt und Charaktereigenschaften der Probanden wurden dafür verantwortlich gemacht. 50 Probanden wurden für eine Studie der Universität Michigan untersucht. Hierbei spritzte man den Probanden Flüssigkeiten in die Kiefermuskulatur, die in der einen Gruppe als schmerzhaft und in der zweiten Gruppe als nicht schmerzhaft angekündigt wurden. Des Weiteren bekam die eine Gruppe ein Schmerzmittel, die andere Gruppe unbewusst ein Placebopräparat. Zuvor wurden ebenfalls Persönlichkeitsmerkmale bestimmt. Man kam zu dem Ergebnis, dass besonders direkte und stressresistente Probanden auf die Placebos reagierten. Die Auswertung ergab weiterhin, dass hauptsächlich ausgeglichene, aufrichtige und altruistische Menschen stärkere Placeboeffekte in Bezug auf Schmerzlinderung zeigten. Die Probanden, die sich laut Persönlichkeitstest schnell verärgern lassen, zeigten keine ausreichenden Effekte auf eine Scheinbehandlung (Peciña et al. 2013). Jedoch sind 50 Probanden für reproduzierbare Ergebnisse nicht ausreichend.

Eine weitere Studie der McGill- Universität in Montreal untersuchte ebenfalls den Zusammenhang zwischen Placebowirkungen und Persönlichkeitseigenschaften. In ihren Versuchen spritzten die Wissenschaftler den 22 gesunden männlichen Probanden eine NaCl-Lösung in die Beine, was bei den Probanden unangenehme Empfindungen hervorrief. Zuvor wurde den Teilnehmern erzählt, man würde an dem einem Bein eine schmerzlindernde Creme testen und im Vergleich dazu am anderen Bein ein Placebo. Tatsächlich handelte es sich jedoch bei beiden Cremes um einfache Hautcreme ohne schmerzstillenden Wirkstoff. Zusätzlich führten sie einen 
Persönlichkeitstest mit den Probanden durch. Die Probanden, bei denen die angeblich lindernde Creme tatsächlich gegen die Schmerzen half, stellten sich im Persönlichkeitstest als besonders abenteuerlustig heraus. Nach Meinung der Forscher hängt diese Reaktion mit der höheren Empfindlichkeit dieser Personengruppe gegenüber Dopamin zusammen. Dieser Botenstoff wirkt sich vor allem auf das Belohnungszentrum im Gehirn aus und sorgt für das angenehme Gefühl, wenn ein Ziel erreicht ist. Allein der Gedanke daran, dass der Schmerz durch die Creme nachlässt, aktiviere das Belohnungszentrum, welches daraufhin Dopamin ausschütte (Schweinhardt et al. 2009).

Weimer et al. 2015 fanden in 15 der 75 durchgeführten Metaanalysen einen Hinweis auf die Beteiligung des Alters am Placeboeffekt. Doch hierbei zeigten sich kontroverse Ergebnisse. In einigen Analysen wurde ein Zusammenhang zwischen jüngeren Versuchspersonen und einem stärkeren Placeboeffekt detektiert, in anderen Analysen war jedoch ein höheres Lebensalter mit stärkeren Placeboeffekten assoziiert. Des Weiteren wurde lediglich in 3 Metaanalysen eine höhere Placeboantwort bei Frauen erkannt.

Diese durchaus kontroversen Ergebnisse im Vergleich zu unserer Studie sollten Anlass für weitere Untersuchungen geben. Es sei noch einmal die geringe Anzahl an Probanden erwähnt. Wenn man weitere ähnliche Placebostudien mit einem größeren Probandenkollektiv durchführen würde, könnten eindeutigere Ergebnisse ermittelt werden. Außerdem ist darauf zu achten, dass die bereits durchgeführten Studien ihr Hauptaugenmerk auf Placeboeffekte in Bezug auf Schmerzlinderung und deren Zusammenhang mit Persönlichkeitsmerkmalen legten. Unsere Studie hingegen untersuchte den Einfluss von Placebo-/Noceboeffekten auf das instrumentelle Lernen.

\subsection{Ausblick und klinische Implikationen}

\subsubsection{Placebos und Lernprozesse}

In der vorliegenden Studie konnte eine Verbesserung von instrumentellen Lernprozessen durch Placeboeffekte nachgewiesen werden. Die Instruktionen, die die Probanden in der Vorbereitungsphase erhielten, dienten der suggestiven Erzeugung von Erwartungen. In der Placebogruppe war diese ausgelöste positive Erwartungshaltung so stark, dass Placeboeffekte zu Lernleistungsverbesserungen führten. In der Nocebogruppe konnten hingegen keine Differenzen der Lernleistung detektiert werden. Eine negative Erwartungshaltung hatte demnach in unserer Studie keinen Einfluss auf die Lernleistung der Probanden. Es sei jedoch auf die geringe Teilnehmerzahl und den Ausschluss weiblicher Versuchsteilnehmer hingewiesen. Demnach wären Folgestudien, in denen auch weibliche Versuchsteilnehmer zugelassen sind, wünschenswert. Die Tatsache, dass verbale Suggesti- 
onen Lernleistungsverbesserungen auslösen können, sollte als Grundlage für weitere Studien mit einer höheren Probandenpopulation genutzt werden. Die Erkenntnisse der aktuellen Studie können auch in den Alltag integriert werden. Sollte es für Lehrer möglich sein, allein durch ihre positive Wortwahl Lernverbesserungen bei ihren Schülern zu evozieren, wäre dies eine Bereicherung für die pädagogische Methodik.

In der aktuellen Studie wurden die Teilnehmer nicht darüber in Kenntnis gesetzt, dass es lediglich Placebogruppen gibt. Im Großteil der klinischen Studien, welche sich meist mit der Neuzulassung von neuen Medikamenten auseinandersetzen, werden jedoch aus ethischen Gründen die Probanden darauf hingewiesen, dass auch die Möglichkeit besteht, dass man der Placebogruppe angehört. Es konnte bereits nachgewiesen werden, dass Probanden, die sich der Möglichkeit bewusst sind, es könne sich um ein Placebo handeln, geringere Placeboeffekte aufweisen, als diejenigen, die glauben, dass es nur eine Verumgruppe gibt (Pollo et al. 2001; Kirsch und Rosadino 1993). In der aktuell vorgestellten Studie gingen alle Probanden davon aus, Verum- tDCS und Infrarotlaserstimulationen zu erhalten. Hierin könnte die Ursache für die durchaus erfolgreiche Placebostimulation liegen. Weitere Studien zu ähnlicher Thematik sollten diesen Verdacht noch verifizieren. Folgestudien, in denen die Probanden darüber in Kenntnis gesetzt werden, dass es Placebo- und Verumgruppen gibt, wären von großem Interesse, um feststellen, ob sich dann die Placebowirkung verringert. AuBerdem könnte man als weitere Möglichkeit einer Folgestudie alle Probanden darüber in Kenntnis setzen, dass sie lediglich mit Sham- tDCS und fake- Infrarotlaserstimulation stimuliert werden, diese Sham- Stimulationen jedoch bereits Erfolge in der Verbesserung der Lernleistung erzielten. Mit diesem Open- Label- Design könnte überprüft werden ,ob verbale Suggestionen allein ebenso erfolgreich sein können, wie eine Kombination aus verbaler Manipulation und einer Scheinbehandlung.

\subsubsection{Placebos und Symptomverbesserung}

In klinischen Studien zur Neuzulassung von Medikamenten treten häufig signifikante Placebo- und Noceboeffekte auf, teilweise mit ähnlicher Wirkungsintensität wie die des Verumpräparates. Infolgedessen wird zunehmend infrage gestellt, ob eine Placebokontrollgruppe tatsächlich die geeignetste Kontrollmethode darstellt. Symptomverbesserungen in der Placebogruppe können auch auf Spontanremission (Kienle und Kiene 1997; Benedetti 2008) zurückzuführen sein. Künftige Studien sollten neben der Verum- und Placebogruppe ebenfalls über eine No- Treatment- Kontrollgruppe verfügen. Somit können bessere Vergleiche auf bedeutend höherem wissenschaftlichen Niveau stattfinden. Insgesamt sollten Placebo- und Noceboeffekte in klinischen Studien zur Neuzulassung von Medikamenten möglichst verringert werden. 
In der täglichen medizinischen Praxis hingegen, sind Placeboeffekte durchaus erwünscht, während Noceboeffekte möglichst vermieden werden sollten. Im Praxisalltag können die positiven Eigenschaften der Placeboeffekte häufig nicht vollständig ausgeschöpft werden, da ethische Limitationen bestehen. Patienten werden über den Einsatz von Placebos während einer Behandlung meist im Unklaren gelassen. Es konnte in der Vergangenheit bereits in einer geringen Anzahl von Studien nachgewiesen werden, dass auch eine offene Placebogabe einen therapeutischen Benefit bewirken kann (Park und Covi 1965; Kaptchuk et al. 2010; Kelley et al. 2012; Kam-Hansen et al. 2014). Es sollten jedoch in Zukunft weitere klinische Studien mit größerem Patientenpool stattfinden, um die Ergebnisse zu verifizieren. Sollte in weiteren Studien der Erfolg der offenen Placebogabe bestätigt werden, könnte dies eine gute, ethisch vertretbare Zusatztherapie im Praxisalltag darstellen. Placebos allein werden Krankheiten nicht vollständig heilen können. Sie können jedoch zu einer Dosisreduktion ohne verminderte Wirksamkeit bei einer kombinierten Placebo - Verum- Therapie führen beziehungsweise leichte Schmerzen durch Placebo- Monotherapie lindern.

Aufklärungen zu möglichen Nebenwirkungen von Medikamenten oder Therapien, wie man sie zum Beispiel in Beipackzetteln von Medikamenten findet, können in Patienten eine negative Erwartungshaltung provozieren, welche ihrerseits einen Noceboeffekt auslösen kann. Die Noceboeffekte führen häufig zu einer verringerten Compliance der Patienten, was gesundheitliche Risiken zur Folge haben kann (Colloca und Finniss 2012; Wells und Kaptchuk 2012). Es ist essentiell wichtig, dass sich Behandler ausreichend Zeit für ihre Patienten nehmen. Eine umfassende Aufklärung unter Betonung der positiven Effekte kann negative Erwartungen verringern und somit die Adhärenz und Wirksamkeit einer Behandlung positiv beeinflussen. Somit könnten Behandlungskosten effizient gesenkt werden und Patienten einen gesundheitlichen Benefit erlangen. 


\section{$5 \quad$ Zusammenfassung}

Placebo-/Noceboeffekte und die zugrunde liegenden Mechanismen sind aktuell Gegenstand intensiver Forschung. Es konnten jedoch bis zum jetzigen Zeitpunkt noch nicht alle Hintergründe vollständig durchleuchtet werden. Zahlreiche Studien beschäftigten sich in der Vergangenheit mit dem Einfluss von Placeboeffekten auf Schmerz und Depressionen. Zum Aufbau einer Erwartungshaltung werden häufig Placebos in Form von Tabletten oder Salben verwendet. Unsere Studie hingegen machte es sich zur Aufgabe, die Beeinflussung des instrumentellen Lernens durch Placebo- und Noceboeffekte zu untersuchen. Zum Aufbau einer Erwartungshaltung erhielten die Probanden keine Tabletten, sondern eine verbale Manipulation. 32 männliche, gesunde Probanden wurden an jeweils zwei aufeinander folgenden Versuchstagen untersucht. Es gab eine Placebo- und eine Nocebogruppe. Zur Überprüfung des instrumentellen Lernverhaltens wurde eine probabilistische Lern- und Entscheidungsfindungsaufgabe verwendet. Den Probanden der Placebogruppe wurde im Vorfeld erklärt, dass die folgende tDCS und Infrarotlaser- Stimulation die kognitiven Leistungen verbessert. Den Teilnehmern der Nocebogruppe erklärte man hingegen, dass die Stimulationen die kognitiven Leistungen beeinträchtigen werden. Somit wurden gezielt differente Erwartungshaltungen aufgebaut. Unterdessen erhielten alle Probanden anstatt der angekündigten tDCS und Infrarotlaser- Stimulation lediglich Sham- Stimulationen. Des Weiteren wurde der Zusammenhang zwischen Persönlichkeitseigenschaften und der Placebowirkung untersucht. Persönlichkeitseigenschaften wurden zuvor anhand von BIS/BAS- und LOT-R- Fragebögen ermittelt. Es konnte eine signifikante Verbesserung des instrumentellen Lernens in der Placebogruppe ermittelt werden. Das Lernverhalten der Nocebogruppe hingegen wurde nicht geändert. Des Weiteren konnte kein Zusammenhang zwischen Persönlichkeitsmerkmalen und der Stärke von Placebo-/Noceboeffekten ermittelt werden. In der Vergangenheit konnten jedoch auch Noceboeffekte in Placebostudien sowie ein Zusammenhang zwischen Persönlichkeitsmerkmalen und der Stärke von Placeboeffekten nachgewiesen werden. Hierbei ist zu beachten, dass das Probandenkollektiv in vorherigen Studien auch aus älteren, kranken Menschen sowie Frauen bestand. In der aktuell vorgestellten Studie wurden jedoch lediglich gesunde männliche Probanden untersucht. Hier könnte eine Ursache für die differenten Ergebnisse liegen. In Zukunft sollten weitere ähnliche Studien durchgeführt werden. Mit einer größeren Probandenanzahl können somit vergleichbarere Ergebnisse geliefert werden, die ihrerseits eventuell einen weiteren Fortschritt in der Aufklärung des noch immer nicht vollständig verstandenen Placebophänomens liefern könnten. 


\section{Anhang}

Anlage 1

\begin{tabular}{|c|c|}
\hline $\begin{array}{c}\text { Abteilung Klinische Neurophysiologie, Medizinische } \\
\text { Fakultät, Universität Göttingen }\end{array}$ & $\begin{array}{c}\text { UNIVERSITÄTSMEDIZIN } \\
\text { GÖTTINGEN }\end{array}$ \\
\hline Robert-Koch-Str. 40, 37070 Göttingen, Tel.: 0551/396650, Telefax: 0551/398126 \\
Prof. Dr. med. W. Paulus
\end{tabular}

\section{Einweisung über die Stimulation}

Diese sichere und nicht-invasive Stimulationskombination wurde bereits erfolgreich zur Steigerung von Lernund kognitiven Fähigkeiten in zahlreichen vorangegangenen Studien angewendet. Dort wurde die Effektivität der Stimulation bestätigt. Durch die Stimulationskombination entsteht eine vorläufige, aber deutliche Steigerung der menschlichen Kognitionsfähigkeiten. Der Effekt der Stimulation hält ca. 30 Minuten an, kognitive Fähigkeiten werden ungefähr um 10 bis 25 Prozent erhöht. Dies gilt auch für das Treffen von Entscheidungen sowie das statistische Lernen.

Während dieser Sitzung erhalten Sie eine Stimulation von einer Minute. Sie werden die Stimulation direkt vor Beginn der Aufgabe erhalten. Am Anfang und während der gesamten Dauer der Stimulation spüren die meisten Probanden ein leichtes Jucken oder Kribbeln unter den Elektroden. Bitte denken Sie daran, dass Ihre Augen während der gesamten Dauer der Stimulation geschlossen sein sollen. 


\begin{tabular}{|c|c|}
\hline $\begin{array}{c}\text { Abteilung Klinische Neurophysiologie, Medizinische } \\
\text { Fakultät, Universität Göttingen }\end{array}$ & $\begin{array}{r}\text { UNIVERSITÄTSMEDIZIN } \\
\text { GÖTTINGEN }\end{array}$ \\
\hline Robert-Koch-Str. 40, 37070 Göttingen, Tel.: 0551/396650, Telefax: 0551/398126 \\
Prof. Dr. med. W. Paulus
\end{tabular}

\section{Einweisung über die Stimulation}

Es wurde in vorangegangenen Studien gezeigt, dass diese sichere und nicht-invasive Stimulationskombination Lern- und kognitive Fähigkeiten vermindert. Dort wurden die negativen Effekte dieser Stimulation bestätigt. Durch die Stimulationskombination entsteht ein vorläufiger, aber deutlicher Verlust der menschlichen Kognitionsfähigkeiten. Der Effekt der Stimulation hält ca. 30 Minuten an, kognitive Fähigkeiten werden ungefähr um 10 bis 25 Prozent verringert. Dies gilt auch für das Treffen von Entscheidungen sowie das statistische Lernen.

Während dieser Sitzung erhalten Sie eine Stimulation von einer Minute. Sie werden die Stimulation direkt vor Beginn der Aufgabe erhalten. Am Anfang und während der gesamten Dauer der Stimulation spüren die meisten Probanden ein leichtes Jucken oder Kribbeln unter den Elektroden. Bitte denken Sie daran, dass Ihre Augen während der gesamten Dauer der Stimulation geschlossen sein sollen. 


\begin{tabular}{|c|c|}
\hline $\begin{array}{c}\text { Abteilung Klinische Neurophysiologie, Medizinische } \\
\text { Fakultät, Universität Göttingen }\end{array}$ & $\begin{array}{c}\text { UNIVERSITÄTSMEDIZIN } \\
\text { GÖTTINGEN }\end{array}$ \\
\hline Robert-Koch-Str. 40, 37070 Göttingen, Tel.: 0551/396650, Telefax: 0551/398126 \\
Prof. Dr. med. W. Paulus
\end{tabular}

\section{Einweisung über die Stimulation}

Gestern hat die Stimulation Ihre Leistung erheblich verbessert, daher haben wir dieses Mal die Aufgabe für Sie noch schwieriger gemacht. Heute werden Sie die gleiche Stimulation wie gestern bekommen und versuchen Sie bitte auch dieses Mal wieder so gut wie möglich zu sein. Vergessen Sie nicht, Sie erhalten 1 Cent nach jeder korrekten Entscheidung. 


\begin{tabular}{|c|c|}
\hline $\begin{array}{c}\text { Abteilung Klinische Neurophysiologie, Medizinische } \\
\text { Fakultät, Universität Göttingen }\end{array}$ & $\begin{array}{c}\text { UNIVERSITÄTSMEDIZIN } \\
\text { GÖTTINGEN }\end{array}$ \\
\hline Robert-Koch-Str. 40, 37070 Göttingen, Tel.: 0551/396650, Telefax: 0551/398126 \\
Prof. Dr. med. W. Paulus
\end{tabular}

\section{Einweisung über die Stimulation}

Gestern hat die Stimulation Ihre Leistung deutlich vermindert, daher haben wir dieses Mal die Aufgabe für Sie ein wenig einfacher gemacht. Heute werden Sie die gleiche Stimulation wie gestern bekommen und versuchen Sie bitte auch dieses Mal wieder so gut wie möglich zu sein. Vergessen Sie nicht, Sie erhalten 1 Cent nach jeder korrekten Entscheidung. 
Anlage 2

Universitätsmedizin Göttingen, D-37099 Göttingen

Zentrum Neurologische Medizin, Abteilung Klinische Neurophysiologie

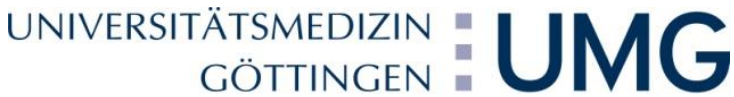

Zentrum Neurologische Medizin Abteilung Klinische Neurophysiologie

Direktor: Prof. Dr. W. Paulus

D-37099 Göttingen Briefpost Robert-Koch-Straße 40, D-37075 Göttingen Adresse +49 (0)55139- $6650 / 8461$ Telefon +49(0)55139- 8126 Fax

Prof. Dr. med. Walter Paulusl wpaulus@med.uni-goettingen.de

+49 (0)551 39 - 6710 Telefon Neurologische Station +49 (0)55139- 10363 Telefon Epilepsie-Monitoring-Einheit +49 (0)55139- 13669 Fax

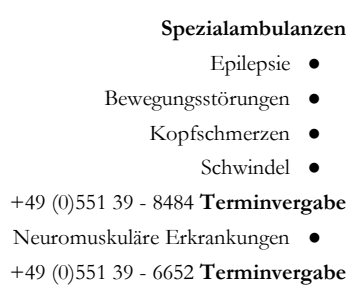

Aktenzeichen

Titel der Studie

\section{Einfluss transkranieller Stromstimulation auf die kognitiven Funktionen}

\section{A) Implizites Lernen - Studie 3}

Sehr geehrte Damen und Herren,

wir möchten Sie bitten, an einer wissenschaftlichen Untersuchung teilzunehmen. Durch diese Studie entsteht Ihnen kein direkter Nutzen, die Ergebnisse können aber möglicherweise in der Zukunft geeignet sein, neue therapeutische Verfahren zur Behandlung von Erkrankungen zu entwickeln. Wissenschaftliche Studien sind notwendig, um Erkenntnisse über die Wirksamkeit und Verträglichkeit von neuen Methoden zu gewinnen oder zu erweitern. Die Studie, die wir Ihnen hier vorstellen, wurde von der zuständigen Ethikkommission zustimmend bewertet und genehmigt. Diese Studie wird in der Abteilung klinische Neurophysiologie, Göttingen durchgeführt; es sollen insgesamt 60 Personen untersucht werden. Die Studie wird veranlasst, organisiert und finanziert durch die Abteilung klinische Neurophysiologie, Universitätsmedizin Göttingen. 
Ihre Teilnahme an dieser Studie ist freiwillig. Sie werden in diese Studie also nur dann einbezogen, wenn Sie dazu schriftlich Ihre Einwilligung erklären. Selbstverständlich ist es Ihnen jederzeit möglich, ohne Angaben von Gründen und ohne dass Ihnen hieraus Nachteile entstehen, von der Teilnahme an dieser Studie zurückzutreten. Sie wurden bereits auf die geplante Studie angesprochen. Der nachfolgende Text soll Ihnen die Ziele und den Ablauf erläutern. Anschließend wird ein Prüfarzt das Aufklärungsgespräch mit Ihnen führen. Bitte zögern Sie nicht, alle Punkte anzusprechen, die Ihnen unklar sind. Sie werden danach ausreichend Bedenkzeit erhalten, um über Ihre Teilnahme zu entscheiden. Da die Teilnahme an der Studie für Sie mit zusätzlichem Aufwand verbunden ist, bitten wir Sie, die nachfolgende Aufklärung sorgfältig zu lesen und danach zu entscheiden, ob Sie an der Studie teilnehmen wollen oder nicht.

\section{Warum wird diese Studie durchgeführt?}

Das Ziel unserer Studie ist die Wirksamkeit der Strom- und Infrarotlaserstimulation auf das Lernen zu charakterisieren. Mit diesem Wissen wird zum einen ein besseres wissenschaftliches Verständnis der Signalübertragung im Gehirn gewonnen, zum anderen kann das therapeutische Potenzial dieser Methodik besser beurteilt werden.

\section{Wie ist der Ablauf der Studie und was muss ich bei der Teilnahme beachten?}

Diese Studie umfasst 2 Sitzungen, in denen der Einfluss schwachen Stroms und Laser, der durch die Kopfhaut und den Schädel (transkraniell) eine Erregbarkeitsänderung der oberflächlichen Gehirnschichten erzeugen kann, auf das Erlernen einer Aufgabe untersucht werden soll.

Die erste Sitzung umfasst 2 Phasen: die erste Phase ist eine Baseline-Phase, während dieser Phase werden Sie die Aufgabe haben, aber keine Stimulation bekommen. Die zweite Phase wird mit Strom- und Infrarotlaserstimulation von einer Minute durchgeführt. Sie werden die Stimulation direkt vor Beginn der Aufgabe erhalten.

Die zweite Sitzung umfasst eine Phase, und wird mit Strom- und Infrarotlaserstimulation von einer Minute durchgeführt. Während dieser Phase werden Sie die Aufgabe haben.

Am Ende jeder Sitzungen sollen Sie einen Fragebogen ausfüllen. In diesem Fragebogen wird nach Ihrer körperlichen Verfassung vor der Stimulation, sowie nach Ihren Empfindungen während und nach der Stimulation gefragt.

Jede Sitzung dauert etwa 90 Minuten, die Sitzungen müssen an zwei aufeinanderfolgenden Tagen stattfinden.

Die Aufgabe besteht aus 2 Abschnitten. Im ersten Abschnitt werden Sie auf einem Monitor vor sich verschiedene Bildpaare sehen. Ihre Aufgabe besteht darin das richtige Bild (mit einer höheren Chance korrekt zu sein) zu wählen. Nach jeder Entscheidung erhalten Sie eine Rückmeldung “-;" oder ":-" die Ihnen angibt, ob Ihre Entscheidung korrekt war. Hierbei ist wichtig, dass es keine absolut richtige Antwort gibt, einige Symbole 
haben jedoch eine höhere Chance korrekt zu sein. Das lächelnde Gesicht, “-)”, steht für eine leistungsabhängige Vergütung von 0,01 Euro, während das traurige Gesicht, “:)", keine zusätzliche Vergütung erbringt (0 Euro).

Der zweite Abschnitt beginnt 10 Minuten nach Beendigung des ersten Abschnitts. Diesmal sehen Sie verschiedene Kombinationen der Bilder und müssen weiterhin wählen welches die bessere Option ist. Wichtig ist hierbei, dass Sie weiterhin 0,01 Euro für jede korrekte Antwort erhalten, allerdings erhalten sie keine Rückmeldung zu Ihrer Entscheidung.

\section{Welchen persönlichen Nutzen habe ich von der Teilnahme an der Studie?}

Persönliche Nutzen sind durch diese Untersuchung nicht zu erwarten.

\section{Welche Risiken sind mit der Teilnahme an der Studie verbunden?}

Dieses Verfahren wurde bereits in mehreren Untersuchungen angewendet und hat sich als risiko- und nebenwirkungsarm erwiesen, wenn die Ausschlusskriterien beachtet werden (siehe unten). Belastungen sind gering. Der Strom ist für Sie nicht oder allenfalls sehr geringfügig wahrnehmbar. Allenfalls in seltenen Fällen ist mit Auftreten von Müdigkeit und Kopfschmerzen zu rechnen. Schwerwiegende Nebenwirkungen sind unter Beachtung der Ausschlusskriterien ebenfalls nicht zu erwarten. Bitte teilen Sie den Mitarbeitern der Prüfstelle alle Beschwerden, Erkrankungen oder Verletzungen umgehend, ggf. telefonisch mit, die im Verlauf der Studie auftreten.

\section{Wer darf an dieser Studie nicht teilnehmen?}

Falls einer der folgenden Punkte auf Sie zutrifft, ist eine Teilnahme an der Studie nicht möglich:

- $\quad$ Alter $<18$ oder $>30$

- Hinweise auf eine chronische oder Residuen (Reste) einer neurologischen Erkrankung (Erkrankung des Nervensystems) in der Vorgeschichte.

- Herzschrittmacher, Tiefe Hirnstimulation

- Metallimplantate im Kopfbereich (in den Kopfbereich eingesetztes Metall, z.B. Clips nach Operation eines intrazerebralen Aneurysmas (Gefäßaussackung im Bereich der Gehirngefäße), Implantation eine künstlichen Hörschnecke)

- Intrazerebrale Ischämien (Mangeldurcbblutung des Gebirns/Schlaganfall)/Blutungen in der Vorgeschichte.

- Hinweise auf epileptische Anfälle in der Vorgeschichte.

- Schädelhirntrauma mit Bewusstseinsverlust in der Vorgeschichte

- Vorliegen einer gravierenden internistischen (Erkrankung der inneren Organe) oder psychiatrischen Vorerkrankung (seelische Erkrankung)

- Alkohol-, Medikamenten- und Drogenabhängigkeit

- Rezeptive oder globale Aphasie (Störung des Sprachverständnisses bzw. zusätzllich des Sprechens)

- Gleichzeitige Teilnahme an einer anderen wissenschaftlichen Studie

6. Entstehen für mich Kosten durch die Teilnahme an der Studie? Erhalte ich eine Aufwandsentschädigung? 
Durch die Teilnahme an dieser wissenschaftlichen Studie entstehen für Sie keine zusätzlichen Kosten. Die Vergütung beträgt 5 Euro pro angefangener Stunde. Zusätzlich erhalten Sie nach jeder korrekten Antwort eine leistungsabhängige Vergütung in Höhe von 0,01 Euro pro korrekter Antwort. Sie können Ihre absolute Vergütung somit durch korrekte Antworten erhöhen. Bei Studienabbruch wird Ihnen die Aufwandserstattung dann anteilig ausgezahlt.

\section{Bin ich während der wissenschaftlichen Studie versichert?}

Bei der Studie sind alle Studienteilnehmer durch die Haftpflichtversicherung des Uni-Klinikums versichert. Wenn Sie vermuten, dass durch die Teilnahme an der wissenschaftlichen Studie Ihre Gesundheit geschädigt wurde, müssen Sie unverzüglich den Prüfarzt informieren.

\section{Kann meine Teilnahme an der Studie vorzeitig beendet werden?}

Sie können jederzeit, auch ohne Angabe von Gründen, Ihre Teilnahme beenden. Unter gewissen Umständen ist es aber auch möglich, dass der Prüfarzt entscheidet, Ihre Teilnahme an der Studie vorzeitig zu beenden, ohne dass Sie auf die Entscheidung Einfluss haben. Die Gründe hierfür können z. B. sein:

- Ihre weitere Teilnahme an der Studie ist ärztlich nicht mehr vertretbar;

- es wird die gesamte Studie abgebrochen.

\section{Was geschieht mit meinen Daten?}

Ihre Daten werden in unserer Abteilung auf zwei verschiedenen Arten gespeichert: Personenbezogene Daten (Name, Geburtsdatum, Gewicht, Geschlecht, Telefonnummer und Adresse) werden in einer Kartei gespeichert. Die Daten ihrer Untersuchung werden getrennt davon aufbewahrt. Diese werden pseudonymisiert gespeichert, d.h. ohne Namensnennung, sondern nur mit einer Nummer codiert. Die Zuordnung der Daten zu einer Person ist nur möglich, wenn hierfür der Schlüssel eingesetzt wird, mit dem die Daten pseudonymisiert wurden. Eine Entschlüsselung ist nur durch die verantwortlichen Studienleiter möglich. Dritte erhalten keinen Einblick in Ihre Originalunterlagen. Die Daten werden nicht weitergegeben und verlassen nicht die Abteilung. Die personenbezogenen Daten werden 10 Jahre aufbewahrt. Selbstverständlich ist es Ihnen jederzeit möglich, ohne Angaben von Gründen und ohne dass Ihnen hieraus Nachteile entstehen, von der Teilnahme an dieser Studie zurückzutreten. Sie haben die Möglichkeit, Auskunft über die gespeicherten Daten zu erhalten. Sie können Ihr Einverständnis zur Speicherung personenbezogener Daten jederzeit widerrufen. Im Falle des Widerrufs werden alle gespeicherten personenbezogenen Daten gelöscht.

\section{An wen wende ich mich bei weiteren Fragen?}

Sie haben stets die Gelegenheit zu weiteren Beratungsgesprächen mit dem auf Seite 1 genannten oder einem anderen Prüfarzt. 


\section{Anlage 3}

Anweisung. Der folgende Fragebogen enthält eine Reihe von Feststellungen, mit denen man sich selbst beschreiben kann. Diese Feststellungen können genau auf Sie zutreffen, eher zutreffen, eher nicht oder gar nicht auf Sie zutreffen. Zur Beantwortung des Fragebogens setzen Sie ein Kreuz in den entsprechenden Kreis. Bitte beantworten Sie jede Feststellung, auch wenn Sie einmal nicht sicher sind, welche Antwort für Sie zutrifft. Kreuzen Sie dann diejenige Antwort an, die noch am ehesten auf Sie zutrifft.

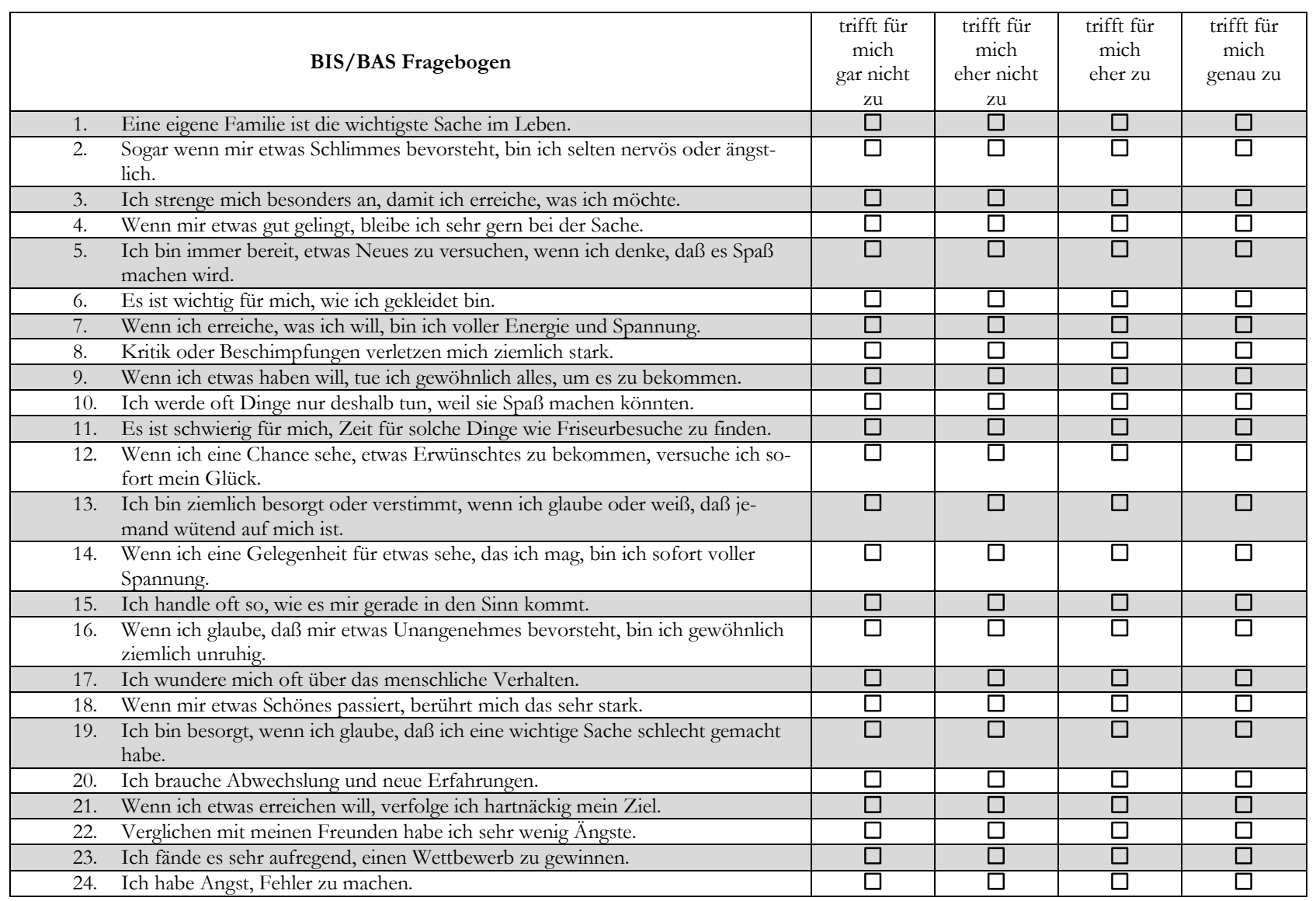


Anlage 4

Edinburgh Handedness Inventory (Oldfield 1971)

Participant ID:

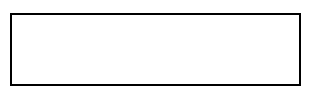

Datum:

Zeigen Sie bitte welche Ihrer Hände Sie bei den folgenden Tätigkeiten bevorzugen, indem Sie in der entsprechenden Spalte ein + eintragen. Wo die Bevorzugung einer Hand so stark ist, dass Sie nie versuchen würden, die andere Hand dafür zu verwenden, es sei denn, Sie würden dazu gezwungen, tragen Sie ein ++ ein. Für den Fall, dass Sie wirklich keine Ihrer Hände bevorzugen, tragen Sie + in beide Spalten ein. Manche Aktivitäten benötigen beide Hände. In diesen Fällen steht der Teil der Aufgabe oder das Objekt, für den bzw. das Sie entscheiden müssen, welche Hand Sie bevorzugen, in Klammern. Bitte versuchen Sie alle Fragen zu beantworten und tragen Sie nur nichts ein, wenn Sie keinerlei Erfahrung mit dem Objekt oder der Aufgabe haben.

\begin{tabular}{|l|l|l|}
\hline & links & rechts \\
\hline schreiben & & \\
\hline zeichnen & & \\
\hline werfen & & \\
\hline Schere & & \\
\hline Zahnbürste & & \\
\hline Messer z.B. beim Brotschneiden & & \\
\hline Löffel & & \\
\hline Besen (obere Hand) & & \\
\hline Streichholz anzünden (Streichholz) & & \\
\hline Schachtel öffnen (Deckel) & & \\
\hline
\end{tabular}

Auswertung (bitte frei lassen)

\section{Summe}

Laterality Quotient (L.Q.) L.Q .= $\left(\sum \mathbf{R}-\sum \mathrm{L}\right) /\left(\sum \mathrm{r}+\sum \mathbf{L}\right) \times 100$ 


\section{Anlage 5}

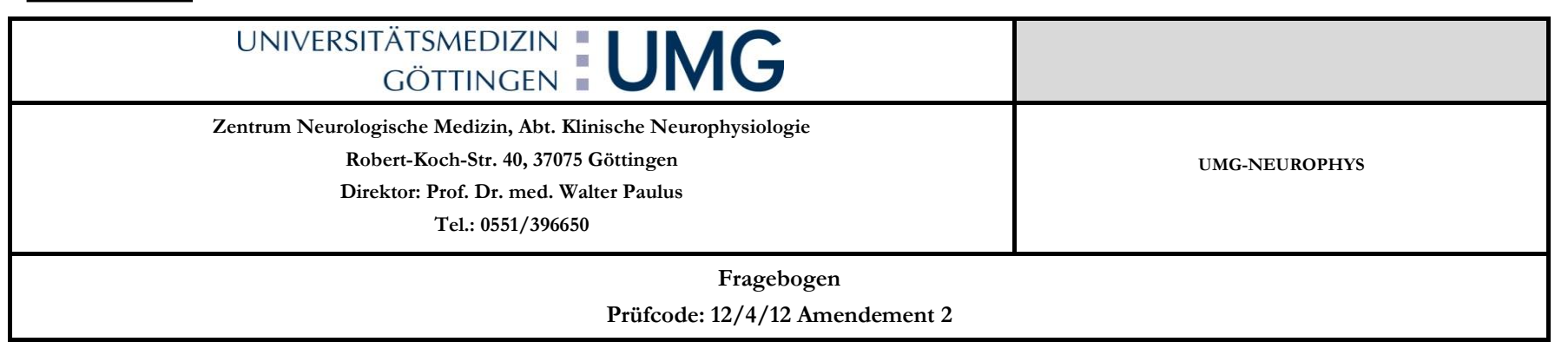

Anweisung. Bitte kreuzen Sie immer die Antwort an.

\begin{tabular}{|ll|l|}
\hline 1. Wie viele Jahre haben Sie mit Schule, Ausbildung und Studium verbracht? (in Jahren): & \\
2. Was studieren Sie bzw. welche Ausbildung haben Sie absolviert? & \\
\hline 3. Haben Sie bereits in einem anderen Experiment mit elektrischer Stimulation teilgenommen? & $\square$ JA $\square$ NEIN \\
\hline 4. Haben Sie bereits in einem anderen Experiment in unserer Abteilung teilgenommen? & $\square$ JA $\square$ NEIN \\
\hline 5. Nehmen Sie zurzeit an einem anderen Experiment Teil? & $\square$ JA $\square$ NEIN \\
\hline Haben Sie Kopfschmerzen? & $\begin{array}{l}\square \text { JA } \square \text { NEIN } \\
\text { Falls ja, wie oft haben Sie Kopfschmerzen? } \\
\square \text { Jeden Tag } \\
\square \text { Mehrmals pro Woche } \\
\square \text { Einmal pro Woche } \\
\square \text { Mehrmals pro Monat } \\
\square \text { Weniger als einmal pro Monat } \\
\text { Bitte geben Sie an, wie intensiv Ihre Kopfschmerzen sind! } \\
\text { (1 = gering - 10 nicht aushaltbar): }\end{array}$ \\
\end{tabular}




\begin{tabular}{|c|c|}
\hline UNIVERSITÄTSMEDIZIN & \\
GÖTTINGEN & Datum (TT/MM/JJJ): \\
\hline $\begin{array}{c}\text { Zentrum Neurologische Medizin, Abt. Klinische Neurophysiologie } \\
\text { Robert-Koch-Str. 40, 37075 Göttingen } \\
\text { Direktor: Prof. Dr. med. Walter Paulus } \\
\text { Tel.: 0551/396650 }\end{array}$ & UMG-NEUROPHYs \\
\hline
\end{tabular}

A)

Anweisung. Bitte kreuzen Sie immer die Antwort an.

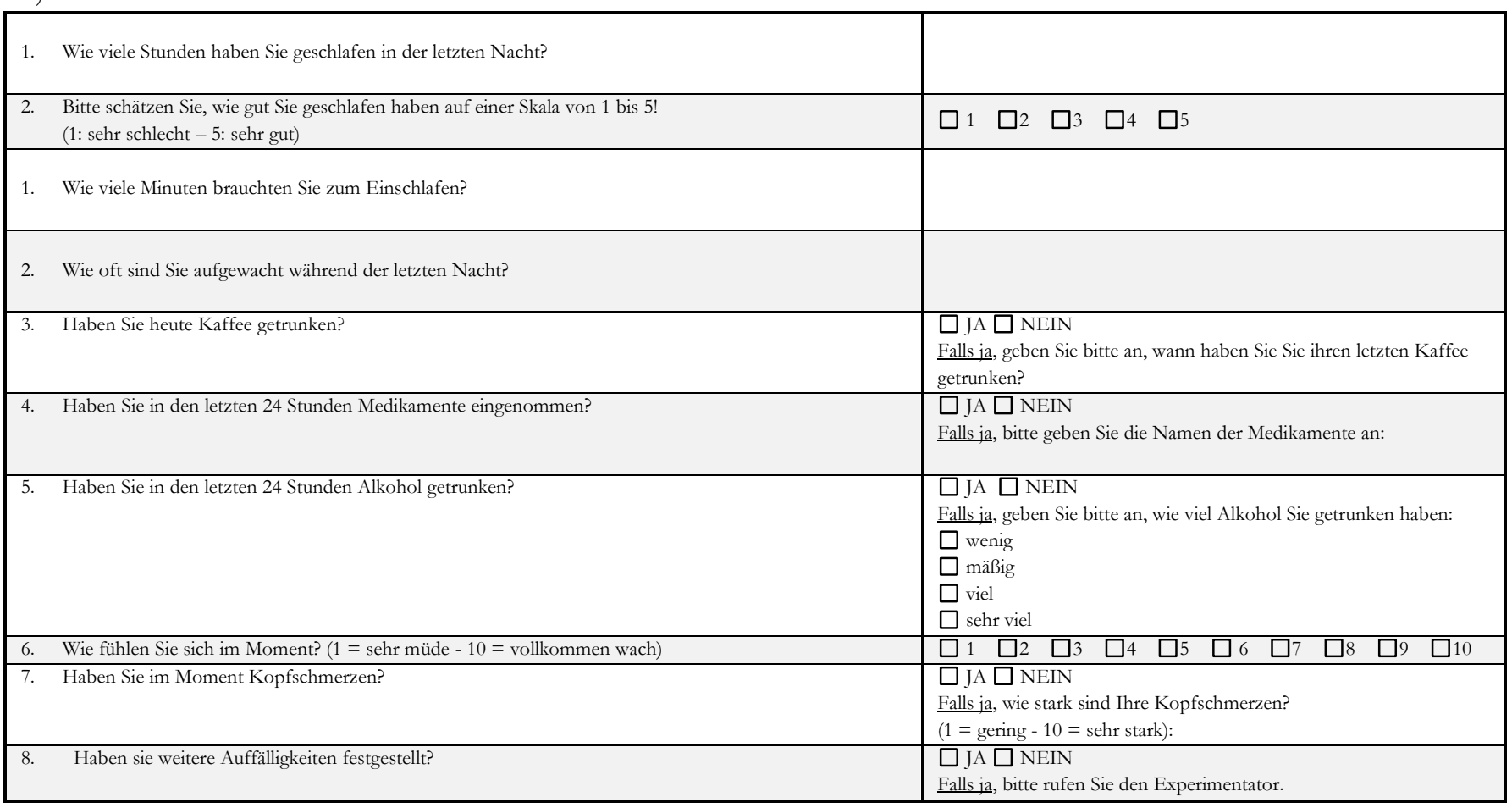

B)

\begin{tabular}{|c|c|c|}
\hline & Was denken Sie, wird die Stimulation Ihre Leistung verändern? & $\begin{array}{l}\text { JA } \square \text { NEIN } \\
\text { Falls ja: } \\
\square \text { VERBESSERN } \\
\square \text { VERSCHLECHTERN } \\
\text { Falls ja, was denken Sie, wie stark wird dieser Effekt in Prozent } \\
\text { sein*? } \\
\begin{array}{|l}\text { *? } \\
\text { * z.B. durch die Stimulation wird sich meine Leistung um } 10 \% \\
\text { verbessern/ verschlechtern) }\end{array} \\
\end{array}$ \\
\hline \multicolumn{3}{|c|}{ C) } \\
\hline & Wie fühlen Sie sich im Moment? $(1=$ sehr müde $-10=$ vollkommen wach $)$ & $\begin{array}{llllllllll}\square & \square 2 & \square 3 & \square^{4} & \square 5 & \square 6 & \square 7 & \square 8 & \square 9 & \square^{10}\end{array}$ \\
\hline & Haben Sie während der Stimulation unter den Elektroden Hautbeschwerden verspürt? & $\begin{array}{l}\square \text { JA } \square \text { NEIN } \\
\text { Falls ja, wie stark waren die Beschwerden? } \\
(1=\text { gering }-10=\text { sehr stark): }\end{array}$ \\
\hline
\end{tabular}




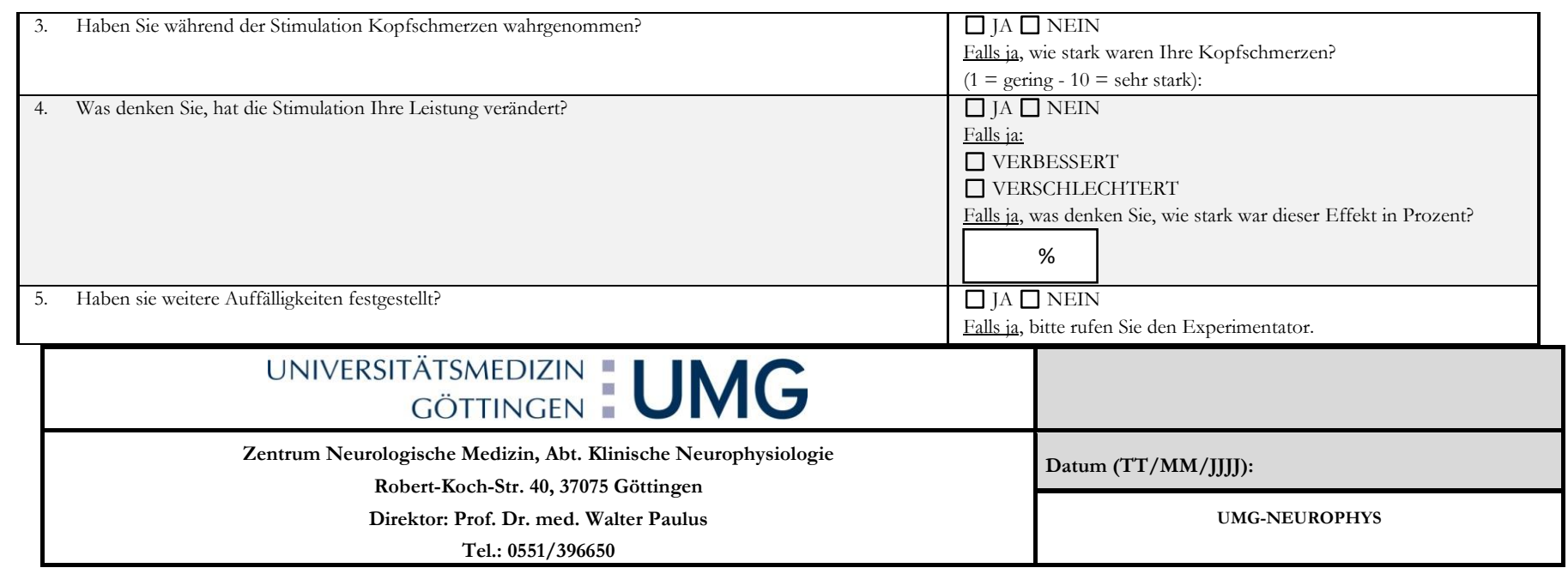

A)

Anweisung. Bitte kreuzen Sie immer die Antwort an.

\begin{tabular}{|c|c|}
\hline 3. Wie viele Stunden haben Sie geschlafen in der letzten Nacht? & \\
\hline $\begin{array}{l}\text { 4. Bitte schätzen Sie, wie gut Sie geschlafen haben auf einer Skala von } 1 \text { bis } 5 \text { ! } \\
\text { (1: sehr schlecht - 5: sehr gut) }\end{array}$ & $\square 1 \quad \square 2 \quad \square 3 \quad \square 4 \quad \square 5$ \\
\hline 9. Wie viele Minuten brauchten Sie zum Einschlafen? & \\
\hline 10. Wie oft sind Sie aufgewacht während der letzten Nacht? & \\
\hline 11. Haben Sie heute Kaffee getrunken? & $\begin{array}{l}\square \text { JA } \square \text { NEIN } \\
\text { Falls ja, geben Sie bitte an, wann haben Sie Sie ihren letzten Kaffee } \\
\text { getrunken? }\end{array}$ \\
\hline 12. Haben Sie in den letzten 24 Stunden Medikamente eingenommen? & $\begin{array}{l}\square \text { JA } \square \text { NEIN } \\
\text { Falls ja, bitte geben Sie die Namen der Medikamente an: }\end{array}$ \\
\hline 13. Haben Sie in den letzten 24 Stunden Alkohol getrunken? & $\begin{array}{l}\square \text { JA } \square \text { NEIN } \\
\text { Falls ja, geben Sie bitte an, wie viel Alkohol Sie getrunken haben: } \\
\square \text { wenig } \\
\square \text { mäßig } \\
\square \text { viel } \\
\square \text { sehr viel }\end{array}$ \\
\hline 14. Wie fühlen Sie sich im Moment? $(1=$ sehr müde $-10=$ vollkommen wach $)$ & \begin{tabular}{|llllllllll}
$\square$ & $\square 2$ & $\square 3$ & $\square 4$ & $\square 5$ & $\square 6$ & $\square 7$ & $\square 8$ & $\square 9$ & $\square 10$
\end{tabular} \\
\hline 15. Haben Sie im Moment Kopfschmerzen? & $\begin{array}{l}\square \text { JA } \square \text { NEIN } \\
\text { Falls ja, wie stark sind Ihre Kopfschmerzen? } \\
(1=\text { gering }-10=\text { sehr stark): }\end{array}$ \\
\hline 16. Haben sie weitere Auffälligkeiten festgestellt? & $\begin{array}{l}\square \text { JA } \square \text { NEIN } \\
\text { Falls ja, bitte rufen Sie den Experimentator. }\end{array}$ \\
\hline
\end{tabular}

B)

2. Was denken Sie, wird die Stimulation Ihre Leistung verändern?

$\square$ JA $\square$ NEIN

Falls ja:

$\square$ VERBESSERN

$\square$ VERSCHLECHTERN

$\underline{\text { Falls ja, }}$, was denken Sie, wie stark wird dieser Effekt in Prozent sein*?

$\%$

* ( z.B. durch die Stimulation wird sich meine Leistung um $10 \%$ verbessern/ verschlechtern) 
C)

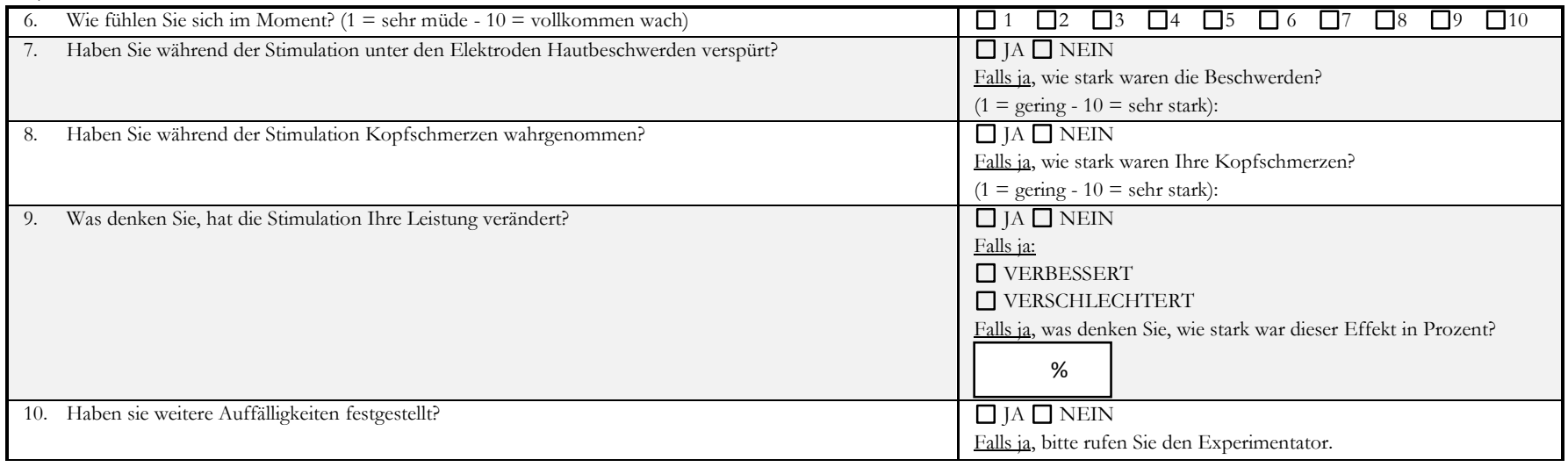




\section{Anlage 6}

Anweisung: Bitte geben Sie an, inwieweit Ihre Meinung mit den folgenden Aussagen übereinstimmt.

\begin{tabular}{|c|c|c|c|c|c|}
\hline LOT-R Fragebogen & $\begin{array}{c}\text { trifft } \\
\text { ausgesprochen } \\
\text { zu }\end{array}$ & $\begin{array}{c}\text { trifft } \\
\text { etwas } \\
\text { zu }\end{array}$ & teils/teils & $\begin{array}{l}\text { trifft } \\
\text { kaum } \\
\text { nicht }\end{array}$ & $\begin{array}{c}\text { trifft } \\
\text { überhaupt } \\
\text { nicht }\end{array}$ \\
\hline 1. Auch in ungewissen Zeiten erwarte ich normalerweise das Beste. & $\square$ & $\square$ & $\square$ & $\square$ & $\square$ \\
\hline 2. Es fällt mir leicht, mich zu entspannen. & $\square$ & $\square$ & $\square$ & $\square$ & $\square$ \\
\hline 3. Wenn bei mir etwas schief laufen kann, dann tut es das auch. & $\square$ & $\square$ & $\square$ & $\square$ & $\square$ \\
\hline 4. Meine Zukunft sehe ich immer optimistisch. & $\square$ & $\square$ & $\square$ & $\square$ & $\square$ \\
\hline 5. In meinem Freundeskreis fühle ich mich wohl. & $\square$ & $\square$ & $\square$ & $\square$ & $\square$ \\
\hline 6. Es ist wichtig für mich, ständig beschäftigt zu sein. & $\square$ & $\square$ & $\square$ & $\square$ & $\square$ \\
\hline 7. Fast nie entwickeln sich die Dinge nach meinen Vorstellungen. & $\square$ & $\square$ & $\square$ & $\square$ & $\square$ \\
\hline 8. Ich bin nicht allzu leicht aus der Fassung zu bringen. & $\square$ & $\square$ & $\square$ & $\square$ & $\square$ \\
\hline 9. Ich zähle selten darauf, dass mir etwas Gutes widerfährt. & $\square$ & $\square$ & $\square$ & $\square$ & $\square$ \\
\hline $\begin{array}{l}\text { 10. Alles in allem erwarte ich, dass mir mehr gute als schlechte Dinge } \\
\text { widerfahren. }\end{array}$ & $\square$ & $\square$ & $\square$ & $\square$ & $\square$ \\
\hline
\end{tabular}




\begin{tabular}{|c|r|r|}
\hline Anlage 7 & $\begin{array}{c}\text { Abteilung Klinische Neurophysiologie, } \\
\text { Medizinische Fakultät, Universität Göttin- } \\
\text { gen }\end{array}$ & $\begin{array}{r}\text { UNIVERSITÄTSMEDIZIN } \\
\text { GÖTTINGEN }\end{array}$ \\
\cline { 2 - 3 } & \multicolumn{2}{|c|}{$\begin{array}{c}\text { Robert-Koch-Str. 40, 37070 Göttingen, Tel.: 0551/396650, Telefax: 0551/398126 } \\
\text { Prof. Dr. med. W. Paulus }\end{array}$} \\
\hline
\end{tabular}

\section{Einweisung - Lernphase}

\section{Die Symbole}

Es werden Ihnen gleichzeitig ein Paar (=zwei chinesische Schriftzeichen; im Folgenden als Schriftzeichen bezeichnet) von 3 Paaren auf dem Bildschirm präsentiert. Eines der gezeigten Schriftzeichen von jedem Paar stellt eine 'richtige' Antwort dar, das andere eine 'falsche'.

\section{Welche ist die richtige Antwort?}

Sie wissen anfangs jedoch nicht, welches Schriftzeichen falsch oder richtig ist! Es gibt keine AUSSCHLIESS-

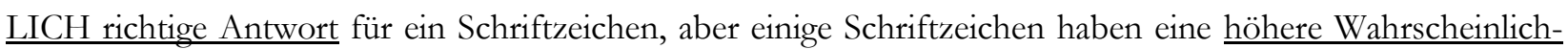
keit, die korrekte Antwort zu sein.

\section{Ihre Aufgabe}

Ihre Aufgabe ist es, durch Ausprobieren herauszufinden, welches Schriftzeichen eine höhere Wahrscheinlichkeit hat, korrekt zu sein. Versuchen Sie dasjenige Schriftzeichen zu wählen, bei dem Sie eine korrekte Antwort für am wahrscheinlichsten halten. Die Schriftzeichen werden sowohl auf der linken als auch der rechten Seite dargestellt, aber die Seite hat keinen Einfluss auf die Richtigkeit des Schriftzeichens.

\section{Wie kann ich das Symbol von dem Paar auswäblen?}

Drücken Sie die Taste L, um das Schriftzeichen auf der linken Seite zu wählen und Taste R für das rechte Schriftzeichen (Figur 1). Es könnte am Anfang etwas verwirrend erscheinen, aber es gibt genügend Zeit zu üben und sich damit vertraut machen!

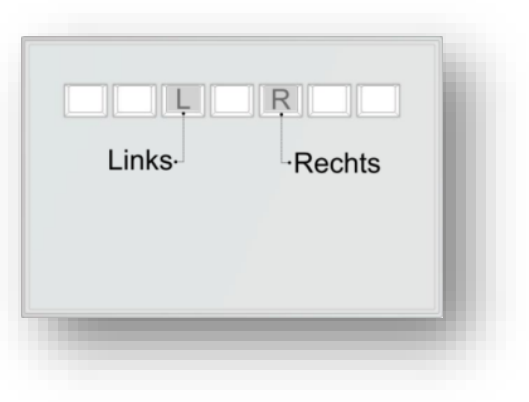


Figur 1.

\section{Rückmeldung nach jeder Entscheidung}

Nach jeder Entscheidung erhalten Sie eine Rückmeldung (Figur 2). Das grüne, lächelnde Gesicht, “(;), steht für eine leistungsabhängige Vergütung von 1 Cent, während das rote, traurige Gesicht, “®”, keine zusätzliche Vergütung erbringt (0 Cent). Bitte vergessen Sie nicht, es gibt keine ausschließlich richtige Antwort für ein Schriftzeichen. Also, bei dieser Aufgabe können Sie auch manchmal für eine eigentlich 'falsche' Antwort Geld bekommen (nämlich das grüne, lächelnde Gesicht), und Sie können auch nach einer eigentlich 'richtigen' Antwort keine zusätzliche Vergütung bekommen (nämlich das rote, traurige Gesicht). Dennoch gibt es für jedes Paar ein Schriftzeichen, welches vorteilhafter ist als das andere. Dies ist der Fall, weil ein Schriftzeichen von jedem Paar eine höhere Wahrscheinlichkeit hat, die korrekte Antwort zu sein.

Das letzte, gelbe Gesicht steht für eine späte Antwort. Sie haben 1,7 Sekunden, um eine der Tasten zu betätigen. Wenn Sie bis zum Ende der 1,7 Sekunden keine Taste drücken, bekommen Sie die gelbe Rückmeldung (d.h. keine zusätzliche Vergütung).
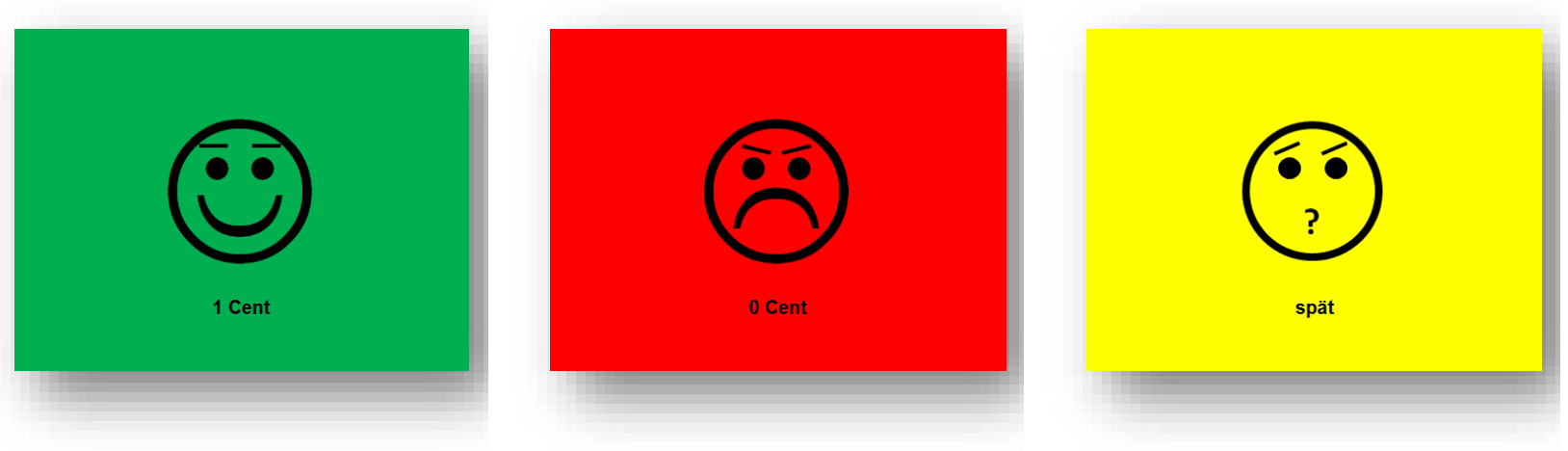

Figur 2.

\section{Haben Sie noch Fragen?}

Falls Ihnen vor Beginn Fragen auftreten sollten, stellen Sie diese gerne. Wenn Sie keine Fragen mehr haben, werden wir mit einem kurzen Fragebogen und dann mit der Übungseinheit beginnen, welche ca. 2 Minuten dauern wird. Das Ziel dieser Einheit ist, sicherzugehen, dass Sie während des Experiments mit dem Programm vertraut sind.

\section{Die Bilder}

Als nächstes werden Sie die Schriftzeichen sehen, die im Experiment verwendet werden. Das Ziel ist es, mit den Schriftzeichen vertraut zu werden. Jedes der Schriftzeichen werden Sie 5 Sekunden lang zweimal hintereinander sehen. Bitte sehen Sie die Schriftzeichen aufmerksam an, Sie brauchen sich aber keine kleinen Details zu merken. 


\begin{tabular}{|c|r|}
\hline $\begin{array}{c}\text { Abteilung Klinische Neurophysiologie, Medizinische } \\
\text { Fakultät, Universität Göttingen }\end{array}$ & $\begin{array}{r}\text { UNIVERSITÄTSMEDIZIN } \\
\text { GÖTTINGEN }\end{array}$ \\
\hline \multicolumn{2}{|c|}{ Robert-Koch-Str. 40, 37070 Göttingen, Tel.: 0551/396650, Telefax: $0551 / 398126$} \\
Prof. Dr. med. W. Paulus
\end{tabular}

\section{Einweisung - Testphase}

Jetzt ist es an der Zeit zu testen, was Sie gelernt haben. Während dieses Versuches werden Sie KEINE Rückmeldung (z.B.: ":)" oder “(:)") für ihre Auswahl erhalten. Wenn Sie eine unbekannte Schriftzeichenkombination sehen, entscheiden Sie sich für das, das Sie persönlich als „,richtiger“ empfinden, basierend auf dem, was Sie in den vorangegangenen Sitzungen gelernt haben. Wenn Sie sich nicht sicher sind, welches Sie nehmen sollen, hören Sie einfach auf ihr Gefühl. Sie erhalten 1 Cent nach jeder korrekten Entscheidung. Die Testphase dauert ca. 5 Minuten. 


\section{Anlage 8}

\begin{tabular}{|c|c|}
\hline UNIVERSITÄTSMEDIZIN \\
GÖTTINGEN \\
\hline $\begin{array}{c}\text { Zentrum Neurologische Medizin, Abt. Klinische Neurophysiologie } \\
\text { Robert-Koch-Str. 40, 37075 Göttingen } \\
\text { Direktor: Prof. Dr. med. Walter Paulus } \\
\text { Tel.: 0551/396650 }\end{array}$ \\
UMG-NEUROPHYS \\
Fragebogen vor der Aufgabe \\
Prüfcode: $12 / 4 / 12$ Amendement 2 \\
\hline
\end{tabular}

Anweisung. Bitte kreuzen Sie immer die Antwort an.

1. Wenn ich diese Rückmeldungsoptionen sehe:

A)

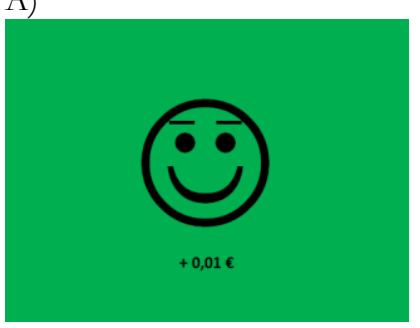

Ich verdiene Geld.

Ich bekomme kein Geld.

Ich habe spät geantwortet.
B)

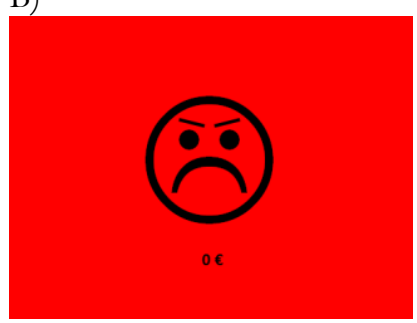

$\square$ Ich verdiene Geld.

$\square$ Ich bekomme kein Geld.

Ich habe spät geantwortet.
C)

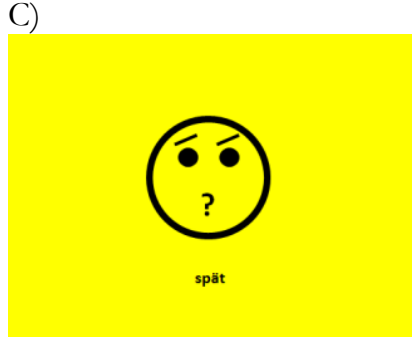

Ich verdiene Geld.

Ich bekomme kein Geld.

Ich habe spät geantwortet.

2. Wenn ich die richtige Antwort wähle (das chinesische Schriftzeichnen mit höherer Wahrscheinlichkeit zum Geld zu verdienen), bekomme ich immer die korrekte Rückmeldung:

Die Aussage ist richtig.

$\square$ Die Aussage ist falsch.

3. $\mathrm{Ob}$ das chinesische Schriftzeichnen links oder rechts erscheint hat keinen Einfluss auf die Richtigkeit:

$\square$ Die Aussage ist richtig.

$\square$ Die Aussage ist falsch. 


\section{$7 \quad$ Literaturverzeichnis}

Ambrus GG, Al-Moyed H, Chaieb L, Sarp L, Antal A, Paulus W (2012): The fade-in-short stimulation-fade out approach to sham tDCS-reliable at $1 \mathrm{~mA}$ for naive and experienced subjects, but not investigators. Brain Stimul $\underline{5}(4), 499-504$

Beecher HK (1955): The powerful placebo. J Am Med Assoc 159(17), 1602-1606

Benedetti F (2008): Mechanisms of placebo and placebo-related effects across diseases and treatments. Annu Rev Pharmacol Toxicol 48, 33-60

Benedetti F, Pollo A, Lopiano L, Lanotte M, Vighetti S, Rainero I (2003): Conscious expectation and unconscious conditioning in analgesic, motor, and hormonal placebo/nocebo responses. J Neurosci $\underline{23}(10), 4315-4323$

Benedetti F, Mayberg HS, Wager TD, Stohler CS, Zubieta JK (2005): Neurobiological mechanisms of the placebo effect. J Neurosci 25(45), 10390-10402

Benedetti F, Arduino C, Costa S, Vighetti S, Tarenzi L, Rainero I, Asteggiano G (2006): Loss of expectation-related mechanisms in Alzheimer's disease makes analgesic therapies less effective. Pain $\underline{121}(1), 133-144$

Binz U : Das Placebo-Phänomen. Med. Diss. Mannheim 1977

Boggio PS, Ferrucci R, Rigonatti SP, Covre P, Nitsche M, Pascual-Leone A, Fregni F (2006): Effects of transcranial direct current stimulation on working memory in patients with Parkinson's disease.

J Neurol Sci 249(1), 31-38

Breidert M, Hofbauer K (2009): Placebo: Missverständnisse und Vorurteile. Dtsch Arztebl 106(46), $751-755$ 
Brown WA (1994): Placebo as a treatment for depression. Neuropsychopharmacology 10(4), 265-269

Cheng CP, Chan SS, Mak AD, Chan WC, Cheng ST, Shi L, Lam LCW (2015): Would transcranial direct current stimulation (tDCS) enhance the effects of working memory training in older adults with mild neurocognitive disorder due to Alzheimer's disease: study protocol for a randomized controlled trial. Trials $\underline{16}(1), 1$

Clark PI, Leaverton PE (1994): Scientific and ethical issues in the use of placebo controls in clinical trials. Annu Rev Publ Health 15(1), 19-38Colagiuri B, Boakes RA (2010): Perceived treatment, feedback, and placebo effects in double-blind RCTs: an experimental analysis. Psychopharmacol 208(3), 433-441

Cocco G (2009): Erectile dysfunction after therapy with metoprolol: the Hawthorne effect. Cardiology $\underline{112(3), 174-177}$

Colagiuri B, Boakes RA (2010): Perceived treatment, feedback and placebo effects in double-blind RCTs: an experimental analysis. Psychopharmacol 208(3), 433-441

Colagiuri B, Livesey EJ, Harris JA (2011): Can expectancies produce placebo effects for implicit learning?. Psychon Bull Rev 18(2), 399-405

Colloca L, Benedetti F (2007): Nocebo hyperalgesia: how anxiety is turned into pain. Cur Opin Anesthesiol 20(5), 435-439

Colloca L, Finniss D (2012): Nocebo effects, patient-clinician communication, and therapeutic outcomes. JAMA $\underline{307}(6), 567-568$

Cutler NR, Heller AH, Kurtz NM, Sramek JJ, Lynch TE, Zolnouni PP, Sawin, S (1991): Idioventricular rhythm: hypervagotonia associated with placebo. Ann Pharmacother 25(5), 471-472 
Di Blasi Z, Harkness E, Ernst E, Georgiou A, Kleijnen J (2001): Influence of context effects on health outcomes: a systematic review. Lancet $\underline{357(9258), 757-762}$

Dimond EG, Kittle CF, Crockett JE (1960): Comparison of internal mammary artery ligation and sham operation for angina pectoris. AJC $\underline{5}(4), 483-486$

Dreher JC, Schmidt PJ, Kohn P, Furman D, Rubinow D, Berman KF (2007): Menstrual cycle phase modulates reward-related neural function in women. PNAS 104(7), 2465-2470

Edelmann W: Lernpsychologie. 6., vollständig überarbeitete Auflage. Kösel-Verlag, Kempten 2000

Elsenbruch S, Enck P (2015): Placebo effects and their determinants in gastrointestinal disorders. Nat Rev Gastroenterol Hepatol $\underline{12}(8), 472-485$

Enck P, Bingel U, Schedlowski M, Rief W (2013): The placebo response in medicine: minimize, maximize or personalize?. Nat Rev Drug Discov 12 (3), 191-204

Fecteau S, Pascual-Leone A, Zald DH, Liguori P, Théoret H, Boggio PS, Fregni F (2007): Activation of prefrontal cortex by transcranial direct current stimulation reduces appetite for risk during ambiguous decision making. J Neurosci 27(23), 6212-6218

Fertonani A, Ferrari C, Miniussi C (2015): What do you feel if I apply transcranial electric stimulation? Safety, sensations and secondary induced effects. Clin Neurophysiol 126(11), 2181-2188

Finniss DG, Kaptchuk TJ, Miller F, Benedetti F (2010): Biological, clinical, and ethical advances of placebo effects. Lancet $\underline{375}(9715)$, 686-695

Frank MJ, Moustafa AA, Haughey HM, Curran T, Hutchison KE (2007): Genetic triple dissociation reveals multiple roles for dopamine in reinforcement learning. PNAS 104(41), 16311-16316

Fregni F, Boggio PS, Nitsche M, Bermpohl F, Antal A, Feredoes E ,Pascual-Leone A (2005): Anodal transcranial direct current stimulation of prefrontal cortex enhances working memory. Exp Brain Res $\underline{166}(1), 23-30$ 
Gandiga PC, Hummel FC, Cohen LG (2006): Transcranial DC stimulation (tDCS): a tool for double-blind sham-controlled clinical studies in brain stimulation. Clin Neurophysiol 117(4), 845-850

Glaesmer H, Hoyer J, Klotsche J, Herzberg PY (2008): Die deutsche Version des Life-OrientationTests (LOT-R) zum dispositionellen Optimismus und Pessimismus. ZGP 16 (1), 26-31

Gliedman ML, Girardet R, Karlson KE (1965): Gastroduodenal congestion and the ulcer diathesis. Surgery $\underline{58}(4), 638-645$

Hartig J, Moosbrugger H (2003): Die “ARES-Skalen” zur Erfassung der individuellen BIS-und BASSensitivität. J Ind Diff 24(4), 293-310

Harty S, Robertson IH, Miniussi C, Sheehy OC, Devine CA., McCreery S, O'Connell RG (2014): Transcranial direct current stimulation over right dorsolateral prefrontal cortex enhances error awareness in older age. J Neurosci 34(10), 3646-3652

Häuser W, Sarzi- Puttini B, Tölle TR, Wolfe F (2012a): Placebo und Nocebo responses in randomised controlled trials of drugs applying for approval for fibromyalgia syndrome treatment: systematic review and meta- analysis. Clin Exp Rheumatol 30 (6 Suppl 74), 78-87

Häuser W, Hansen E, Enck P (2012b): Nocebo Phänomene in der Medizin: Ihre Relevanz in der alltäglichen klinischen Praxis. Dtsch Arztebl Int $\underline{109}$ (26),459

Hong G, Lee JC, Robinson JT, Raaz U, Xie L, Huang NF, Dai H (2012): Multifunctional in vivo vascular imaging using near-infrared II fluorescence. Nat Med 18(12), 1841-1846

Horing B, Weimer K, Muth ER, Enck P (2014): Prediction of placebo responses: a systematic review of the literature. Front Psychol $\underline{5}, 1079$

Horvath JC, Forte JD, Carter O (2015): Quantitative review finds no evidence of cognitive effects in healthy populations from single-session transcranial direct current stimulation (tDCS). Brain Stimul $\underline{8}(3), 535-550$ 
Jewett DL, Fein G, Greenberg MH (1990): A double-blind study of symptom provocation to determine food sensitivity. N Engl J Med $\underline{323}(7), 429-433$

Jocham G, Klein TA, Ullsperger M (2011): Dopamine-mediated reinforcement learning signals in the striatum and ventromedial prefrontal cortex underlie value-based choices. J Neurosci 31(5), 16061613

Kam-Hansen S, Jakubowski M, Kelley JM, Kirsch, I, Hoaglin DC, Kaptchuk TJ, Burstein R (2014): Altered placebo and drug labeling changes the outcome of episodic migraine attacks. Sci transl med $\underline{6}(218), 218 \mathrm{ra} 5-218 \mathrm{ra} 5$

Kaminski G, Neisser U: Kognition. In: Dorsch F, Häcker H, Stapf, KH (Hrsg): Dorsch Psychologisches Wörterbuch.; 12. Auflage, Hans Huber Verlag; Bern 1994, S. 387

Kapfhammer JP (2000): Plastizität im Nervensystem, Essay, Quelle: http://www.spektrum.de/lexikon/neurowissenschaft/plastizitaet-im-nervensystem/9979; Zugriff am 18.04.2016

Kaptchuk TJ, Stason WB, Davis RB (2006): Sham device v inert pill: randomized controlled trial of two placebo treatments. BMJ $\underline{332}(7538), 391-7$

Kaptchuk TJ, Friedlander E, Kelley JM, Sanchez MN, Kokkotou E, Singer JP, Lembo AJ (2010): Placebos without deception: a randomized controlled trial in irritable bowel syndrome. PloS one $\underline{5}(12)$, e 15591

Keitel A, Wojtecki L, Hirschmann J, Hartmann CJ, Ferrea S, Südmeyer M, Schnitzler A (2013a): Motor and cognitive placebo-/nocebo-responses in Parkinson's disease patients with deep brain stimulation. Behav Brain Res 250, 199-205

Keitel A, Ferrea S, Südmeyer M, Schnitzler A, Wojtecki L (2013b): Expectation modulates the effect of deep brain stimulation on motor and cognitive function in tremor-dominant Parkinson's disease. PloS one $\underline{8}(12)$, e81878 
Kelley JM, Kaptchuk TJ, Cusin C, Lipkin S, Fava M (2012): Open-label placebo for major depressive

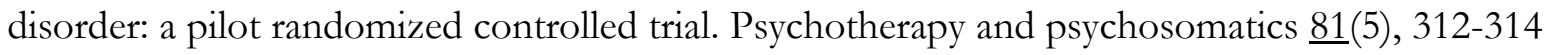

Kennedy WP (1961): The nocebo reaction. MW 95, 203-205

Kentenich H, Pietzner K (2012): Placebo in der Medizin oder: Die Rolle des Arztes im therapeutischen Prozess. J Reprod Med $\underline{8}(2), 15-19$

Kienle GS, Kiene H (1997): The powerful placebo effect: fact or fiction?. J clin epidemiol $\underline{50}(12)$, $1311-1318$

Kincses TZ, Antal A, Nitsche MA, Bártfai O, Paulus W (2004): Facilitation of probabilistic classification learning by transcranial direct current stimulation of the prefrontal cortex in the human. Neuropsychologia $\underline{42}(1), 113-117$

Kirsch I, Rosadino MJ (1993): Do double-blind studies with informed consent yield externally valid results?. Psychopharmacology $\underline{110}(4), 437-442$

Klein TA, Neumann J, Reuter M, Hennig J, von Cramon DY, Ullsperger M (2007): Genetically determined differences in learning from errors. Science $\underline{318}$ (5856), 1642-1645

Klinger R, Soost S, Flor H, Worm M (2007): Classical conditioning and expectancy in placebo hypoalgesia: a randomized controlled study in patients with atopic dermatitis and persons with healthy skin. Pain $\underline{128}(1), 31-39$

Klosterhalfen S, Enck P (2006): Psychobiology of the placebo response. Autonomic Neuroscience $\underline{125}(1), 94-99$

Lang H: Das Arzt-Patient-Verhältnis aus der Sicht der medizinischen Psychologie. In: Schumpelick V, Vogel B (Hrsg): Arzt und Patient - Eine Beziehung im Wandel. Herder, Freiburg i. Br. 2006, 293315 
Liccardi G, Senna G, Russo M, Bonadonna P, Crivellaro M, Dama A, Passalacqua G (2004): Evaluation of the nocebo effect during oral challenge in patients with adverse drug reactions. J Investig Allergol Clin Immunol 14(2), 104-107

Linde K, Allais G, Brinkhaus B, Manheimer E, Vickers A, White AR (2009): Acupuncture for tension-type headache. Cochrane Database Syst Rev 1(1)

Lombardi C, Gargioni S, Canonica GW, Passalacqua G (2008): The nocebo effect during oral challenge in subjects with adverse drug reactions. Eur Ann All Clin Immunol 40(4), 138

Marshall L, Mölle M, Siebner HR, Born J (2005): Bifrontal transcranial direct current stimulation slows reaction time in a working memory task. BMC Neurosci $\underline{6}(1), 23$

Mora MS, Nestoriuc Y, Rief W (2011): Lessons learned from placebo groups in antidepressant trials. Philos Trans R Soc Lond B Biol Sci $\underline{366}(1572), 1879-1888$

Moseley JB, O'Malley K, Petersen NJ, Menke TJ, Brody BA, Kuykendall DH, Wray NP (2002): A controlled trial of arthroscopic surgery for osteoarthritis of the knee. NEJM $\underline{347}(2), 81-88$

Nitsche MA, Paulus W (2000): Excitability changes induced in the human motor cortex by weak transcranial direct current stimulation. J Physiol 527(3), 633-639

Nitsche MA, Paulus W: Transkranielle Gleichstromstimulation. In: Siebner HR, Ziemann U (Hrsg): Das TMS-Buch: Handbuch der transkraniellen Magnetstimulation, Springer-Verlag, Berlin 2007, $533-542$

Nitsche MA, Liebetanz D, Lang N, Antal A, Tergau F, Paulus W (2003a): Safety criteria for transcranial direct current stimulation (tDCS) in humans. Clin Neurophysiol 114(11), 2220- 2222

Nitsche MA, Fricke K, Henschke U, Schlitterlau A, Liebetanz D, Lang N, Paulus W (2003b): Pharmacological modulation of cortical excitability shifts induced by transcranial direct current stimulation in humans. J Physiol 553(1), 293-301 
Nitsche MA, Antal A, Liebetanz D, Lang N, Tergau F, Paulus W (2004): Erzeugung und Modulation von Neuroplastizität durch transkranielle Gleichstromstimulation beim Menschen. Klin Neurophysiol $\underline{35}(02), 55-60$

Nitsche MA, Dömkes S, Karaköse T, Antal A, Liebetanz D, Lang N, Paulus W (2007): Möglichkeiten der Fokussierung der Effekte der transkraniellen Gleichstromstimulation. Klin Neurophysiol $\underline{38}(01)$, P344

Nitsche MA, Cohen LG, Wassermann EM, Priori A, Lang N, Antal A, Pascual-Leone A (2008): Transcranial direct current stimulation: state of the art 2008. Brain Stim 1(3), 206-223

Oldfield RC (1971): The assessment and analysis of handedness: the Edinburgh inventory. Neuropsychologia $\underline{9}(1), 97-113$

Olshansky B (2007): Placebo and nocebo in cardiovascular health: implications for healthcare,research, and the doctor-patient relationship. J Am Coll Cardiol 49(4), 415-21

Park LC, Covi L (1965): Nonblind placebo trial: An exploration of neurotic patients' responses to placebo when its inert content is disclosed. Arch Gen Psychiat 12(4), 336-345

Peciña M, Azhar H, Love TM, Lu T, Fredrickson BL, Stohler CS, Zubieta JK (2013): Personality trait predictors of placebo analgesia and neurobiological correlates. Neuropsychopharmacology $\underline{38}(4), 639-646$

Petersen GL, Finnerup NB, Colloca L, Amanzio M, Price DD, Jensen TS, Vase L ( 2014): The magnitude of nocebo effects in pain: a meta- analysis. Pain 155(8), 1426-1434

Platsch KD (2009): Placebo- Verborgener Schatz der Medizin, Vortrag auf dem Symposium „Heilen“, Benediktushof, Holzkirchen, gehalten am 25. November 2009 , Quelle: http://www.drplatsch.de/tl_files/drplatsch/pdf/Vortrag_Heilende_Essenz_Placebo_End.pdf Zugriff am 01.06.2016 
Pollo A, Amanzio M, Arslanian A, Casadio C, Maggi G, Benedetti F (2001): Response expectancies in placebo analgesia and their clinical relevance. Pain $\underline{93}(1), 77-84$

Poreisz C, Boros K, Antal A, Paulus W (2007): Safety aspects of transcranial direct current stimulation concerning healthy subjects and patients. Brain Res Bull 2⑷, 208- 214

Preston RA, Materson BJ, Reda DJ, Williams DW (2000): Placebo-associated blood pressure response and adverse effects in the treatment of hypertension: observations from a department of veteran's affairs cooperative study. Arch Intern Med 160(10), 1449-54

Price DD, Milling LS, Kirsch I, Duff A, Montgomery GH, Nicholls SS (1999): An analysis of factors that contribute to the magnitude of placebo analgesia in an experimental paradigm. Pain $\underline{83}(2), 147-$ 156

Pschyrembel: Klinisches Wörterbuch. 257. Auflage; de Gruyter Verlag, Berlin 1994, S. 1205

Reid B (2002): The Nocebo Effect: Placebo’s Evil Twin. WP,Ausgabe vom 30. April 2002

Reis J, Schambra HM, Cohen LG, Buch ER, Fritsch B, Zarahn E, Celnix PA, Krakauer JW (2009): Noninvasive cortical stimulation enhances motor skill acquisition over multiple days through an effect on consolidation. Proc Natl Acad Sci USA 106(5), 1590-5

Rief W, Nestoriuc Y, Weiss S, Welzel E, Barsky AJ, Hofmann SG (2009a): Metaanalysis of the placebo response in antidepressant trials. J affect disord $\underline{118}(1), 1-8$

Rief W, Nestoriuc Y, von Lilienfeld-Toal A, Dogan I, Schreiber F, Hofmann SG, Avorn J (2009b): Differences in adverse effect reporting in placebo groups in SSRI and tricyclic antidepressant trials. Drug Safety $\underline{32}(11), 1041-1056$

Rosenzweig P, Brohier S, Zipfel A (1993): The placebo effect in healthy volunteers: influence of experimental conditions on the adverse events profile during phase I studies. Clin Pharmacol Ther 54(5), 578-583 
Scheier MF, Carver CS, Bridges MW (1994): Distinguishing optimism from neuroticism (and trait anxiety, self-mastery, and self-esteem): a reevaluation of the Life Orientation Test.J Pers Soc Psychol $\underline{\text { 67}}(6), 1063$

Schmidt L, Braun EK, Wager TD, Shohamy D (2014): Mind matters: placebo enhances reward learning in Parkinson's disease. Nat Neurosci 17(12), 1793-1797

Schweinhardt P, Seminowicz DA, Jaeger E, Duncan GH, Bushnell MC (2009): The anatomy of the mesolimbic reward system: a link between personality and the placebo analgesic response. J Neurosci $\underline{29}(15), 4882-4887$

Shapiro A, Morris L: The placebo effect in medical and psychological therapies. In: Handbook of Psychotherapy and Behavior Change (2nd Edition), Wiley Verlag, New York 1978, 369-410

Stagg CJ, Nitsche MA (2011): Physiological basis of transcranial direct current stimulation. Neuroscientist 17(1), 37-53

Strobel A, Beauducel A, Debener S, Brocke B (2001): Eine deutschsprachige Version des BIS/BASFragebogens von Carver und White. Zeitschrift für Differentielle und diagnostische Psychologie

Ströhle A (2000): Increased response to a putative panicogenic nocebo administration in female patients with panic disorder. J Psychiatr Res $\underline{34}(6), 439-442$

Tretrop J: Der Placeboeffekt bei chronischen Rückenschmerzpatienten: eine klinisch-experimentelle Studie. Naturwissenschaftliche Diss. Hamburg 2007

van Steenburgh J, Varvaris M, Schretlen D, Vannorsdall T, Gordon B (2016): Bifrontal Transcranial Direct Current Stimulation Enhances Working Memory in Adults with High-Functioning Autism (S9. 001). Neurology $\underline{86}(16), 9001$

Vase L, Robinson ME, Verne G, Price DD (2005): Increased placebo analgesia over time in irritable bowel syndrome (ibs) patients is associated with desire and expectation but not endogenous opioid mechanisms. Pain 115(3), 338-347 
Waber RL, Shiv B, Carmon Z, Ariely D (2008): Commercial features of placebo and therapeutic. JAMA $\underline{299}(9), 1016-7$

Weimer K, Colloca L, Enck P (2015): Age and sex as moderators of the placebo response-an evaluation of systematic reviews and meta-analyses across medicine. Gerontology 61(2), 97-108

Wells RE, Kaptchuk TJ (2012): To tell the truth, the whole truth, may do patients harm: the problem of the nocebo effect for informed consent. Am J Bioeth, 12(3), 22-29

Zwissler B, Sperber C, Aigeldinger S, Schindler S, Kissler J, Plewnia C (2014): Shaping memory accuracy by left prefrontal transcranial direct current stimulation. J Neurosci 34(11), 4022-402 


\section{Danksagung}

Herzlich danken möchte ich meiner Doktormutter Frau apl. Prof. Dr. Andrea Antal für die Überlassung des Themas und die beständige Unterstützung sowie konstruktive Kritik. Ein besonderer Dank gilt auch meinem Betreuer Zsolt Turi (Ph.D.), der sich viel Zeit genommen hat und mich technisch sowie organisatorisch, besonders in der Zeit der Experimente, sehr unterstützt hat. Nicht zuletzt möchte ich mich recht herzlich bei den teilnehmenden Probanden bedanken. 


\section{Lebenslauf}

Mein Name ist Luisa Gunkel. Ich wurde am 07.09.1990 in Heilbad Heiligenstadt geboren. Ich bin wohnhaft in 37318 Uder, Lehmkuhle 4. Ich bin ledig und habe keine Kinder. Von 1997 bis 2000 besuchte ich die Grundschule „Birkenfelde“ in Birkenfelde. 2000 bis 2001 besuchte ich die Grundschule „Im Luttertal“ in Lutter. Von 2001 bis 2009 war ich Schülerin am staatlichen Gymnasium „Johann Georg Lingemann“ in Heilbad Heiligenstadt. Das Abitur habe ich im Juni 2009 bestanden. Von Oktober 2009 bis November 2014 absolvierte ich das Studium der Zahnmedizin an der „Georg- August- Universität“ in Göttingen. Im September 2014 habe ich mit der Bearbeitung meiner Doktorarbeit in der Abteilung „Klinik für Klinische Neurophysiologie“ unter Anleitung von Frau apl. Prof. Dr. Andrea Antal begonnen. Am 27.11.2014 habe ich das Staatsexamen in Zahnmedizin mit der Gesamtnote „sehr gut“ bestanden. Seit 1. Dezember 2014 arbeite ich als Assistenzzahnärztin in der Zahnarztpraxis von Dipl. med. Barbara Wedekind in Uder. 